VILNIUS GEDIMINAS TECHNICAL UNIVERSITY

Dmitrij MELICHOV

\title{
ON ESTIMATION OF THE HURST INDEX OF SOLUTIONS OF STOCHASTIC DIFFERENTIAL EQUATIONS
}

DOCTORAL DISSERTATION

PHYSICAL SCIENCES,

MATHEMATICS (01P)

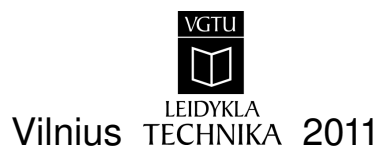


Doctoral dissertation was prepared at Vilnius Gediminas Technical University in 2007-2011.

\section{Scientific Supervisor}

Prof Dr Habil Kęstutis KUBILIUS (Vilnius Gediminas Technical University, Physical Sciences, Mathematics - 01P). 
VILNIAUS GEDIMINO TECHNIKOS UNIVERSITETAS

Dmitrij MELICHOV

\section{APIE STOCHASTINIŲ DIFERENCIALINIŲ LYGČIŲ SPRENDINIŲ HURSTO INDEKSO VERTINIMA}

DAKTARO DISERTACIJA

FIZINIAI MOKSLAI,

MATEMATIKA (01P)

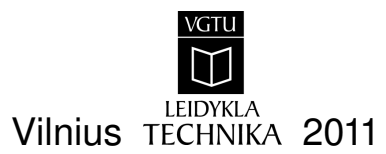


Disertacija rengta 2007-2011 metais Vilniaus Gedimino technikos universitete.

\section{Mokslinis vadovas}

prof. habil. dr. Kęstutis KUBILIUS (Vilniaus Gedimino technikos universitetas, fiziniai mokslai, matematika-01P).

http://leidykla.vgtu.lt

VGTU leidyklos TECHNIKA 1917-M mokslo literatūros knyga

ISBN 978-9955-28-999-9

(C) Dmitrij Melichov, 2011

(C) VGTU leidykla TECHNIKA, 2011 


\section{Abstract}

The main topic of this dissertation is the estimation of the Hurst index $H$ of the solutions of stochastic differential equations (SDEs) driven by the fractional Brownian motion ( $\mathrm{fBm}$ ).

Firstly, the limit behavior of the first and second order quadratic variations of the solutions of SDEs driven by the $\mathrm{fBm}$ is analyzed. This yields several strongly consistent estimators of the Hurst index $H$. Secondly, it is proved that in case the solution of the SDE is replaced by its Milstein approximation, the estimators remain strongly consistent. Additionally, the possibilities of applying the increment ratios (IR) statistic based estimator of $H$ originally obtained by J. M. Bardet and D. Surgailis in 2010 to the fractional geometric Brownian motion are examined. Furthermore, this dissertation derives the convergence rate of the modified Gladyshev's estimator of the Hurst index to its real value.

The estimators obtained in the dissertation were compared with several other known estimators of the Hurst index $H$, namely the naive and ordinary least squares Gladyshev and $\eta$-summing oscillation estimators, the variogram estimator and the IR estimator. The models chosen for comparison of these estimators were the fractional Ornstein-Uhlenbeck $(\mathrm{O}-\mathrm{U})$ process and the fractional geometric Brownian motion $(\mathrm{gBm})$. The initial inference about the behavior of these estimators was drawn for the O-U process which is Gaussian, while the $\mathrm{gBm}$ process was used to check how the estimators behave in a nonGaussian case. The scope of modelling was 100 sample paths of the length $n=2^{14}+1$ for each value of $H \in\{0.55,0.6, \ldots, 0.95\}$ on the unit interval $t \in[0,1]$.

The dissertation consists of the introduction, 3 main chapters, the conclusions, the bibliography, the list of author's publications on the topic of dissertation and two appendices.

The results obtained during the doctoral studies were published in 6 papers in reviewed periodic scientific journals and were presented at 5 conferences, of which 2 - international. 


\section{Santrauka}

Pagrindinė šios disertacijos tema - stochastinių diferencialiniu lygčiu (SDL), valdomų trupmeninio Brauno judesio (tBj), sprendinių Hursto indekso $H$ vertinimas.

Pirmiausia disertacijoje išnagrinèta SDL, valdomu tBj, sprendiniu pirmos ir antros eilès kvadratiniu variaciju ribinè elgsena. Iš šiu rezultatų seka keli stipriai pagrịsti Hursto indekso $H$ įvertiniai. Irodyta, kad šie įvertiniai išlieka stipriai pagrịsti, jei tikra sprendinio trajektorija keičiama jos Milšteino aproksimacija. Taip pat išnagrinètos pokyčiu santykio (increment ratios) statistikos $H$ ivertinio, gauto J. M. Bardeto ir D. Surgailio 2010 m., taikymo trupmeninio geometrinio Brauno judesio Hursto indekso vertinimui galimybès bei nustatytas modifikuoto Gladyševo $H$ ịvertinio konvergavimo ị tikrąją parametro reikšmę greitis.

Gauti ivertiniai palyginti su kai kuriais kitais žinomais Hursto indekso $H$ ivertiniais: naiviais bei mažiausių kvadratų Gladyševo ir $\eta$-sumavimo osciliacijos ìvertiniais, variogramos įvertiniu ir pokyčiu santykio statistikos įvertiniu. Ivertiniu elgsena buvo palyginta trupmeniniam Ornšteino-Ulenbeko (O$\mathrm{U})$ procesui bei trupmeniniam geometriniam Brauno judesiui (gBj). Pradinès išvados buvo padarytos $\mathrm{O}-\mathrm{U}$ procesui, kuris yra Gauso, o $\mathrm{gBj}$ procesas buvo naudojamas patikrinti, kaip šie įvertiniai elgiasi, kai procesas yra ne Gauso. Modeliavimo apimtis buvo po 100 trajektoriju kiekvienai Hursto indekso reikšmei $H \in\{0,55,0,6, \ldots, 0,95\}$ vienetiniame intervale $t \in[0,1]$; kiekvienos trajektorijos ilgis buvo $n=2^{14}+1$ tašku.

Disertaciją sudaro įvadas, 3 pagrindiniai skyriai, išvados, literatūros sąrašas, autoriaus publikacijų disertacijos tema sąrašas ir du priedai.

Doktorantūros studiju metu gauti rezultatai buvo paskelbti 6 straipsniuose recenzuojamuose periodiniuose mokslo leidiniuose ir pristatyti 5 konferencijose, iš kurių 2 - tarptautinès. 


\section{Notation}

\section{Symbols}

$\begin{array}{ll}\mathrm{fBm} & \text { - the fractional Brownian motion } \\ \mathrm{SDE} & \text { - stochastic differential equation } \\ \mathrm{gBm} & \text { - the fractional geometric Brownian motion } \\ \mathrm{O}-\mathrm{U} & \text { - the Ornstein-Uhlenbeck process } \\ \mathbb{N} & \text { - the set of natural numbers } \\ \mathbb{Z} & \text { - the set of integer numbers } \\ \mathbb{R} & \text { - the set of real numbers } \\ \mathbf{E X} & \text { - the expectation of } X \\ \mathrm{MSE}(\mathrm{X}) & \text { - the mean squared error of } X \\ \text { a.s. } & \text { - almost surely } \\ \mathbf{1}_{A} & \text { - the indicator function of the set } A \\ {[x]} & \text { - the integer part of } x \\ V_{n}^{(1)}(X, 2) & - \text { the first order quadratic variation of } X \text { (regular subdivisions) } \\ V_{n}^{(2)}(X, 2) & - \text { the second order quadratic variation of } X \text { (regular subdiv.) } \\ V_{\pi_{n}}^{(1)}(X, 2) & \text { - the first order quadratic variation of } X \text { (irregular subdiv.) } \\ V_{\pi_{n}}^{(2)}(X, 2) & - \text { the second order quadratic variation of } X \text { (irregular subdiv.) }\end{array}$


$v_{p}(f ;[a, b])$ - the $p$-variation of $f$ on the interval $[a, b]$

$\mathcal{W}_{p}([a, b])$ - the set of functions with bounded $p$-variation on $[a, b]$ 


\section{Contents}

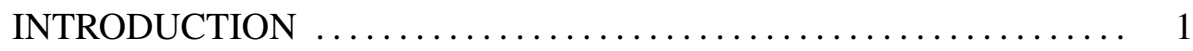

Formulation of the problem $\ldots \ldots \ldots \ldots \ldots \ldots \ldots \ldots \ldots \ldots \ldots, 1$

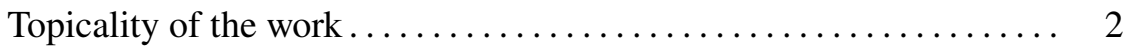

Research object............................... 2

The aim and tasks of the work $\ldots \ldots \ldots \ldots \ldots \ldots \ldots \ldots \ldots \ldots, 2$

Applied methods ................................. 3

Scientific movelty ................................ 3

Practical value of the results ......................... 3

Propositions presented for defence...................... 4

Approval of the results ............................... 4

Structure of the dissertation $\ldots \ldots \ldots \ldots \ldots \ldots \ldots \ldots \ldots \ldots \ldots$

1. DEFINITIONS AND THE HISTORICAL OVERVIEW $\ldots \ldots \ldots \ldots .7$

1.1. The fractional Brownian motion ..................... 7

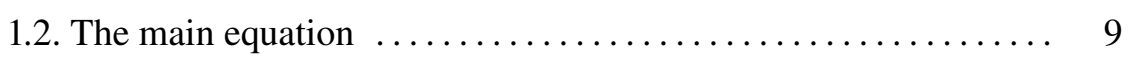

1.3. The estimators of the Hurst index $\ldots \ldots \ldots \ldots \ldots \ldots \ldots \ldots . \ldots$

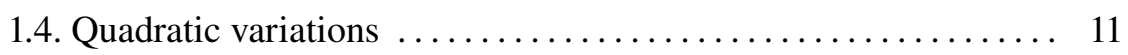

1.4.1. Regular subdivisions $\ldots \ldots \ldots \ldots \ldots \ldots \ldots \ldots \ldots \ldots . \ldots \ldots \ldots$

1.4.2. Irregular subdivisions $\ldots \ldots \ldots \ldots \ldots \ldots \ldots \ldots \ldots, 11$

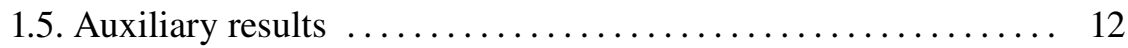

1.6. Conclusions of the first chapter $\ldots \ldots \ldots \ldots \ldots \ldots \ldots \ldots \ldots$ 


\section{QUADRATIC VARIATIONS AND THE INCREMENT RATIO}

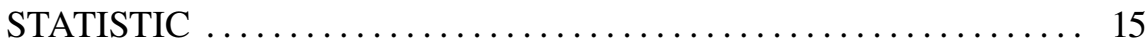

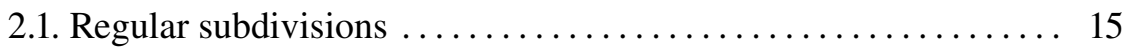

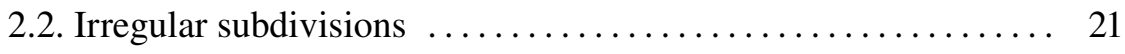

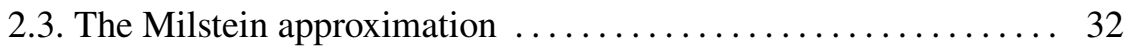

2.4. The increment ratio statistic $\ldots \ldots \ldots \ldots \ldots \ldots \ldots \ldots \ldots \ldots \ldots \ldots$

2.5. The convergence rate of the Gladyshev estimator ......... 42

2.6. Conclusions of the second chapter $\ldots \ldots \ldots \ldots \ldots \ldots \ldots \ldots, 47$

3. MODELLING OF THE ESTIMATORS $\ldots \ldots \ldots \ldots \ldots \ldots \ldots \ldots$

3.1. Generation of the fractional Brownian motion $\ldots \ldots \ldots \ldots \ldots .50$

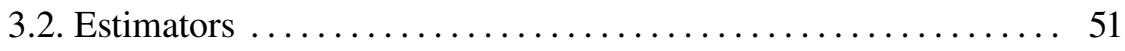

3.2.1. Discrete variation estimators $\ldots \ldots \ldots \ldots \ldots \ldots \ldots \ldots, 51$

3.2.2. Gladyshev and $\eta$-summing oscillation estimators ...... 52

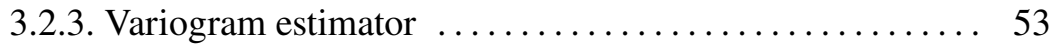

3.2.4. Increment ratios estimator $\ldots \ldots \ldots \ldots \ldots \ldots \ldots \ldots . \ldots \ldots$

3.2. The Ornstein-Uhlenbeck process $\ldots \ldots \ldots \ldots \ldots \ldots \ldots \ldots . \ldots \ldots$

3.2.1. Dependance on the value of the Hurst index .......... 54

3.2.2. Dependance on the length of the sample path ........ 55

3.3. The geometric Brownian motion .................... 56

3.3.1. Dependance on the value of the Hurst index ......... 56

3.3.2. Dependance on the length of the sample path ........ 57

3.4. Conclusions of the third chapter $\ldots \ldots \ldots \ldots \ldots \ldots \ldots \ldots, 58$

GENERAL CONCLUSIONS $\ldots \ldots \ldots \ldots \ldots \ldots \ldots \ldots \ldots \ldots \ldots \ldots$

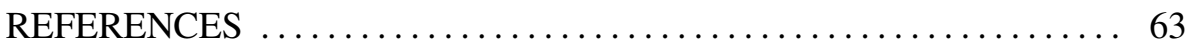

LIST OF AUTHOR'S PUBLICATIONS $\ldots \ldots \ldots \ldots \ldots \ldots \ldots \ldots \ldots$

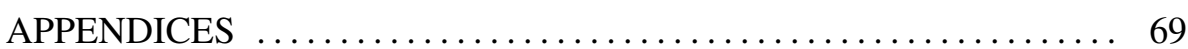

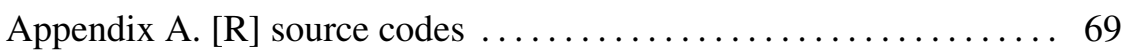

Appendix A.1. Generation of the fractional Brownian motion ... 69

Appendix A.2. Calculation of the estimators ............. 70

Appendix B. Figures $\ldots \ldots \ldots \ldots \ldots \ldots \ldots \ldots \ldots \ldots \ldots \ldots \ldots \ldots$ 


\section{Introduction}

\section{Formulation of the problem}

In fields as diverse as economics and finance, mathematics, physics, chemistry, environmental studies and computer science it is not uncommon to encounter observations made far apart in time or space which are non-trivially correlated. This phenomenon is known as long memory or long-range dependance and was first studied by the hydrologist Hurst (1951) who tried to derive a suitable model for the flow of the Nile river. The stochastic calculus of stochastic processes possessing the long-range dependance property started with the work of Mandelbrot, van Ness (1968) which introduced the fractional Brownian motion ( $\mathrm{fBm}$ ), the backbone of such processes. Later on B. B. Mandelbrot summarized his results on fractals and scaling in Mandelbrot (1995). The first result in which the fBm appeared as the limit of stationary sums of random variables in the Skorokhod topology was obtained by Taqqu (1975). In the 1990s intensive studies of possibilities of applying the $\mathrm{fBm}$ in various teletraffic, finance and climate models started which, in turn, encouraged the stochastic analysis studies of the fBm (e. g., Decreusefond, Üstünel (1995)).

The Hurst index $H \in(0,1)$ determines the correlation structure of $\mathrm{fBm}$ : if $H=1 / 2$ it is the standard Brownian motion, if $H<1 / 2$ the increments of the process are negatively correlated and if $H>1 / 2$ the increments of 
the process are positively correlated which implies the long-range dependance. The latter property of the $\mathrm{fBm}$ encouraged the studies of stochastic models in which the standard Brownian motion is replaced by the $\mathrm{fBm}$, since the longrange dependance is often encountered in the observed data. Therefore it is important to be able to study this dependance and check if it really exists. The problem examined in this thesis is the estimation of the Hurst index $H$ of certain generalizations of the $\mathrm{fBm}$ from discrete data.

\section{Topicality of the work}

The estimation and modeling of the Hurst index has been a subject of intense studies lately. A whole set of methods and estimators have been proposed for the Gaussian processes of the fractional type. However little is known about the construction of the estimators when the considered process is a solution of a stochastic differential equation driven by the $\mathrm{fBm}$. In the work of Berzin, León (2008) such estimators are given for several specific types of such equations, where the integrands are either constants or linear functions. Naturally it's desirable to obtain estimators for the solutions of the general case of stochastic differential equations driven by the $\mathrm{fBm}$ which would be simple to implement and computationally efficient.

\section{Research object}

The research objects are the solutions of stochastic differential equations driven by the fractional Brownian motion with the Hurst index $H>1 / 2$.

\section{The aim and tasks of the work}

The aim of this work is to study the limit behavior of certain statistics based on the observed values of the process and use the obtained results to derive consistent estimators of the Hurst index $H$ as well as to study the properties of these estimators. The tasks of this work are:

1. To study the limit behavior of the quadratic variations of the solutions of SIEs driven by the fBm both in the case of equally and non-equally spaced observations. 
2. To derive consistent estimators of the Hurst index $H$ based on quadratic variations.

3. To study the possibility of applying the increment ratios (IR) statistic to estimate the Hurst index $H$ of the solutions of SIEs driven by the $\mathrm{fBm}$.

4. To compare the performance of the obtained estimators to that of other known estimators.

\section{Applied methods}

In the theoretical part of the work the $p$-variation calculus techniques have been applied along with an array of known inequalities. As for the modelling part of the work, the fractional Brownian motion sample paths were generated using the circular matrix embedding method (see, f.e., Coeurjolly (2000)). All calculations were performed using the R software package.

\section{Scientific novelty}

It was shown that the estimators of the Hurst index $H$ originally obtained by Istas, Lang (1997) and Benassi et al (1998) for the fBm retain their properties when the underlying process is a solution of a stochastic differential equation, which is not necessarily Gaussian. Additionally, it was proved that the IR statistic estimator originally obtained by Bardet, Surgailis (2010) can be used to estimate the Hurst index $H$ of the fractional geometric Brownian motion. Furthermore, the convergence rate of the modified Gladyshev Hurst index estimator has been derived.

\section{Practical value of the results}

The estimators studied in this work are suitable for a wide spectrum of processes including the fractional Ornstein-Uhlenbeck process and the fractional geometric Brownian motion. For the latter two models, the estimators were additionally studied through simulated data. They are easy to implement, computationally efficient and do not impose any specific requirements on the sample path lengths. 


\section{Propositions presented for defence}

1. Two strongly consistent estimators of the Hurst index $H$ of the solution of a stochastic differential equation driven by the fractional Brownian motion have been obtained which is a non-covering extension of the results known up to date.

2. It was shown that the IR statistic estimator of the Hurst index $H$ is applicable to the fractional geometric Brownian motion.

3. The convergence speed of the modified Gladyshev estimator has been obtained.

4. Computer modelling suggests that the performance of the obtained estimators is comparable to or better than that of other estimators considered in this non-exhaustive study.

\section{Approval of the results}

On the topic of dissertation there were 6 papers published in reviewed scientific journals. The research results were reported at 5 scientific conferences. The list of conference talks is as follows:

1. K. Kubilius, D. Melichov, Estimating the Hurst index of the solution of a stochastic integral equation, 10th international Vilnius conference on probability theory and mathematical statistics, Vilnius, 2010.

2. K. Kubilius, D. Melichov, On estimation and asymptotics of the Hurst index of solutions of stochastic integral equations, Applied stochastic models and data analysis, Vilnius, 2009.

3. K. Kubilius, D. Melichov, Using the IR ir DV statistics to estimate the Hurst index of solutions of stochastic differential equations, LMD 52nd conference, Vilnius, 2011.

4. K. Kubilius, D. Melichov, On estimation of the Hurst index of solutions of stochastic integral equations, LMD 51st conference, Šiauliai, 2010.

5. K. Kubilius, D. Melichov, On estimation of the Hurst index of solutions of stochastic integral equations, LMD 50th conference, Vilnius, 2009. 


\section{Structure of the dissertation}

The dissertation consists of the introduction, three chapters, the conclusions, references, the list of author's publications on the topic of the dissertation and two appendices. The total scope of the dissertation is 78 pages, 5 tables, 4 figures and 34 items of reference.

The first chapter is the introduction which presents the considered stochastic differential equation, the overview of other authors' works on the topic of dissertation and introduces some common definitions used further on.

The second chapter presents the obtained theoretical results, namely the asymptotics of the quadratic variations of the solutions of stochastic differential equations driven by the $\mathrm{fBm}$ and the estimators of the Hurst index $H$. Additionally, the usage of the IR statistic based estimator to estimate the Hurst index of the solutions of SDEs is considered; it's proved that if the underlying process is the fractional geometric Brownian motion, then the a.s. convergence of the IR statistic holds. Moreover, the convergence rates of the modified Gladyshev estimator are studied.

The third chapter shows the comparison of performance of the obtained estimators of the Hurst index $H$ with that of other known estimators for a Gaussian (fractional Ornstein-Uhlenbeck) and a non-Gaussian (fractional geometric Brownian motion) processes. 



\section{Definitions and the historical} overview

\subsection{The fractional Brownian motion}

1.1 Definition. A process $B_{t}^{H}=\left\{B_{t}^{H} ; t \geqslant 0\right\}$ is a fractional Brownian motion ( $\mathrm{fBm}$ ) with the Hurst index $H \in(0,1)$ if it is a continuous centered Gaussian process with the covariance function

$$
\mathbf{E}\left(B_{t}^{H} B_{s}^{H}\right)=\frac{1}{2}\left(t^{2 H}+s^{2 H}-|t-s|^{2 H}\right), \quad \forall t, s \geqslant 0 .
$$

The fractional Brownian motion has the following primary properties:

- Self-similarity: for any $a>0$, the processes $\left\{a^{H} B_{t}^{H} ; t \geqslant 0\right\}$ and $\left\{B_{a t}^{H} ; t \geqslant 0\right\}$ have identic probability distributions;

- Stationary increments: for any $s>0$, the processes $\left\{B_{t}^{H} ; t \geqslant 0\right\}$ and $\left\{B_{t+s}^{H}-B_{s}^{H} ; t \geqslant 0\right\}$ have identic probability distributions;

- Correlated increments: for $u<s<t$, the fBm has independent increments only if $H=1 / 2$, this corresponds to the standard Brownian 


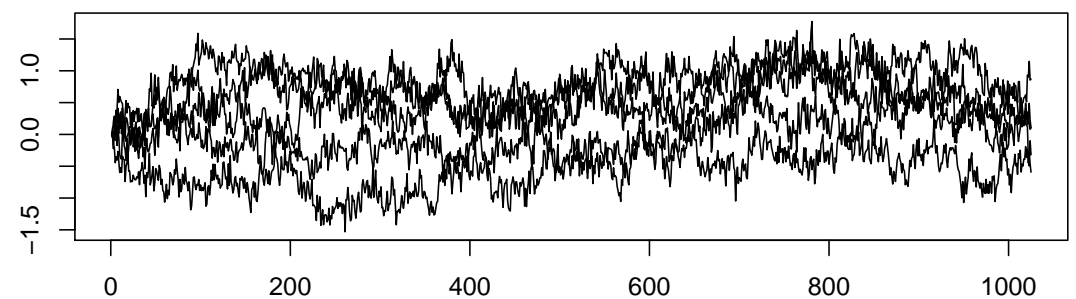

(a) $H=0.2$

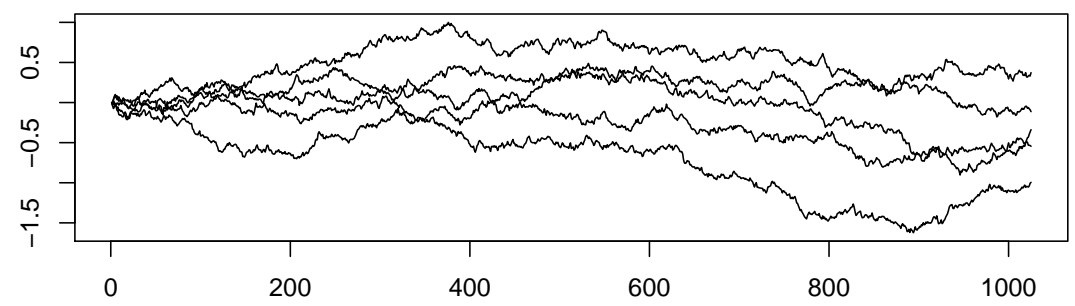

(b) $H=0.5$

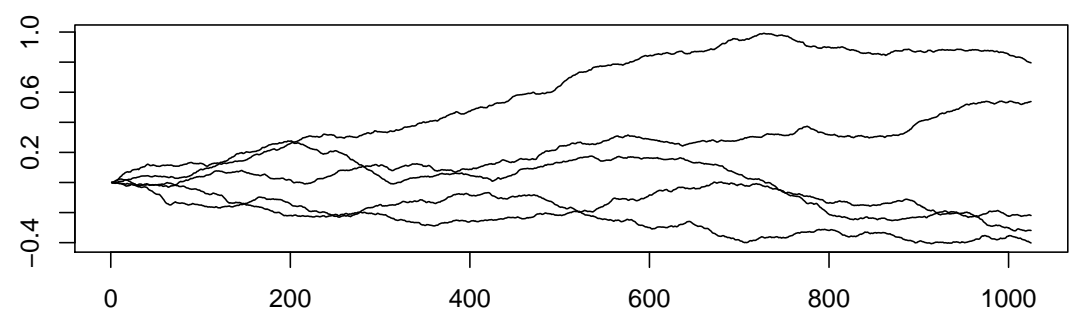

(c) $H=0.8$

Fig. 1.1. 5 sample paths of the fractional Brownian motion, $n=1024$.

motion; if $H \neq 1 / 2$, then the increments of $B^{H}$ are correlated:

$$
\operatorname{corr}\left(B_{t}^{H}-B_{s}^{H}, B_{s}^{H}-B_{u}^{H}\right) \begin{cases}>0 & \text { if } H>1 / 2 \\ =0 & \text { if } H=1 / 2 \\ <0 & \text { if } H<1 / 2\end{cases}
$$

- Hölder continuity: for $\alpha<H$, almost all sample paths of fBm are Hölder continuous of order $\alpha$, that is,

$$
\sup _{t \neq s} \frac{\left|B_{t}^{H}-B_{s}^{H}\right|}{|t-s|^{\alpha}}<\infty .
$$


- Bounded p-variation: almost all sample paths of $\mathrm{fBm}$ with the Hurst index $1 / 2<H<1$ have bounded $p$-variation for $p>1 / H$, that is,

$$
v_{p}\left(B^{H} ;[a, b]\right)=\sup _{\varkappa} \sum_{k=1}^{n}\left|B^{H}\left(t_{k}\right)-B^{H}\left(t_{k-1}\right)\right|^{p}<\infty,
$$

$\varkappa=\left\{t_{i}: i=0, \ldots, n\right\}$ being all finite partitions of the interval $[a, b]$ such that $a=t_{0}<t_{i}<\ldots<t_{n}=b$.

- Fractal dimension: the graph of a sample path of fBm with the Hurst index $H$ has the fractal dimension equal to $2-H$.

\subsection{The main equation}

Consider a SDE driven by the fBm with the Hurst index $1 / 2<H<1$

$$
X_{t}=\xi+\int_{0}^{t} f\left(X_{s}\right) d s+\int_{0}^{t} g\left(X_{s}\right) d B_{s}^{H}
$$

$t \in[0, T], T>0, \xi \in \mathbb{R}$. It is known that almost all sample paths of $B^{H}$, $1 / 2<H<1$, have bounded $p$-variation for $p>1 / H$. Thus the integrals on the right side of (1.1) will exist pathwise as the Riemann-Stieltjes integrals. For $\frac{1}{H}-1<\alpha \leqslant 1, \mathcal{C}^{1+\alpha}(\mathbb{R})$ denotes the set of all $\mathcal{C}^{1}$-functions $g: \mathbb{R} \rightarrow \mathbb{R}$ such that

$$
\sup _{x}\left|g^{\prime}(x)\right|+\sup _{x \neq y} \frac{\left|g^{\prime}(x)-g^{\prime}(y)\right|}{|x-y|^{\alpha}}<\infty .
$$

Let $f$ be a Lipschitz-continuos function and let $g \in \mathcal{C}^{1+\alpha}(\mathbb{R}), \frac{1}{H}-1<$ $\alpha \leqslant 1$. For $1 \leqslant p<1+\alpha$ there exists a unique solution of the equation (1.1) with almost all sample paths in the class of all continuous functions defined on $[0, T]$ with bounded $p$-variation (see Lyons (1994), Dudley (1999), Kubilius (2000), Nualart, Răşcanu (2002)).

For the estimation of the Hurst parameter $H$ first of all we consider the limits of the first and second order quadratic variations of a pathwise solution $X$ of (1.1). Results of such type for Gaussian processes were considered in Bégyn (2005)-Bégyn (2006) (see also references in Bégyn (2006)). 


\subsection{The estimators of the Hurst index}

In 1961, E. Gladyshev derived a limit theorem for a statistic based on the first order quadratic variations of $\mathrm{fBm}$. This yielded an estimator of $H$ which was strongly consistent but not asymptotically normal.

In 1997, another estimator was introduced by J. Istas and G. Lang. This estimator was designed for centered Gaussian processes with stationary increments and again employed the first order quadratic variations. The obtained estimator was asymptotically normal for $H \in(1 / 2,3 / 4)$.

In 1998, A. Benassi et al considered the second order quadratic variations of a class of Gaussian processes, having locally the same fractal properties as the fractional Brownian motion and obtained an estimator of the Hurst index $H$ which was asymptotically normal for all $H$.

In 2001, J. F. Coeurjolly developed a class of consistent estimators of $H$ based on the asymptotic behavior of the $k$-th absolute moment of discrete variations of its sample paths over a discrete partition of the interval [0, 1]. Explicit convergence rates for these types of estimators, valid through the whole range $0<H<1$ of the self-similarity parameter, were derived, and the asymptotic normality of the obtained estimators was established.

In 2005, A. Bégyn considered the second order quadratic variations along general subdivisions for processes with Gaussian increments. A more complete survey on asymptotic behavior of quadratic variations for Gaussian processes can be found in the thesis of A. Bégyn (2006).

In 2006, C. Berzin and J. R. León proposed estimators of $H$ and the diffusion function $g$ for several specific cases of (1.1), namely for the combinations of $f\left(X_{s}\right)=\mu$ or $f\left(X_{s}\right)=\mu X_{s}$ and $g\left(X_{s}\right)=\sigma$ or $g\left(X_{s}\right)=\sigma X_{s}$. Additionally they assumed that the process $X_{t}$ was smoothed by convolution defined as $X_{\varepsilon}(t)=\varphi_{\varepsilon} * X_{t}$ where $\varepsilon$, which tends to zero, is the smoothing parameter and $\varphi_{\varepsilon}(\cdot)$ is the convolution kernel defined as $\varphi_{\varepsilon}(\cdot)=\frac{1}{\varepsilon} \varphi\left(\frac{\dot{\varepsilon}}{\varepsilon}\right)$. Here $\varphi(\cdot)$ is a $\mathcal{C}^{2}$ positive kernel with $L^{1}$ norm equal to one. The estimators of $H$ and $g$ use functionals of the type $\int_{0}^{1} h\left(X_{\varepsilon}(t)\right)\left|\ddot{X}_{\varepsilon}(t)\right|^{k} d t$ where $h(x)=1 /|x|^{k}$ in the case of $g\left(X_{s}\right)=\sigma X_{s}$ and $h(x)=1$ in the case of $g\left(X_{s}\right)=\sigma$.

In 2011, R. Bertrand, M. Fhima and A. Guillin introduced a method for change point analysis on the Hurst index for a piecewise fractional Brownian motion, a generalization of the regular fractional Brownian motion. Their procedure is the combination, on the one hand, of the filtered derivative with $p$ value $(\mathrm{FDpV})$ method for detection of change of the mean, variance or regression parameter, and, on the other hand, of a variation of the increment ratios (IR) statistic estimator introduced in J. M. Bardet and D. Surgailis (2010). 


\subsection{Quadratic variations}

\subsubsection{Regular subdivisions}

1.2 Definition. For a real-valued process $X=\left\{X_{t} ; t \in[0, T]\right\}$, we define the first and second order quadratic variations along regular subdivisions as

$$
V_{n}^{(1)}(X, 2)=\sum_{k=1}^{n}\left(\Delta X_{k}^{n}\right)^{2}, \quad V_{n}^{(2)}(X, 2)=\sum_{k=1}^{n-1}\left(\Delta^{(2)} X_{k}^{n}\right)^{2},
$$

where

$$
\Delta X_{k}^{n}=X\left(t_{k}^{n}\right)-X\left(t_{k-1}^{n}\right), \quad \Delta^{(2)} X_{k}^{n}=X\left(t_{k+1}^{n}\right)-2 X\left(t_{k}^{n}\right)+X\left(t_{k-1}^{n}\right)
$$

and $t_{k}^{n}=k T / n$.

\subsubsection{Irregular subdivisions}

Let $\pi_{n}=\left\{0=t_{0}^{n}<t_{1}^{n}<\cdots<t_{N_{n}}^{n}=T\right\}, T>0$, be a sequence of subdivisions of the interval $[0, T]$ and $\left(N_{n}\right)$ is an increasing sequence of natural numbers. Such sequence of subdivisions is called irregular. Define

$$
m_{n}=\max _{1 \leqslant k \leqslant N_{n}} \Delta t_{k}^{n}, \quad p_{n}=\min _{1 \leqslant k \leqslant N_{n}} \Delta t_{k}^{n}, \quad \Delta t_{k}^{n}=t_{k}^{n}-t_{k-1}^{n} .
$$

Usually the observed values of the process are only available at discrete regular time intervals. However, it may happen that part of the observations are lost, resulting in observations at irregular time intervals.

1.3 Definition. The first and second order quadratic variations of $X$ along the subdivisions $\left(\pi_{n}\right)_{n \in \mathbb{N}}$ with normalization $1 / 2<H<1$ is defined by

$$
V_{\pi_{n}}^{(1)}(X, 2)=\sum_{k=1}^{N_{n}} \frac{\left(\Delta X_{k}^{n}\right)^{2}}{\left(\Delta t_{k}^{n}\right)^{2 H-1}}, \quad \Delta X_{k}^{n}=X\left(t_{k}^{n}\right)-X\left(t_{k-1}^{n}\right),
$$

and

$$
V_{\pi_{n}}^{(2)}(X, 2)=2 \sum_{k=1}^{N_{n}-1} \frac{\Delta t_{k+1}^{n}\left(\Delta_{i r}^{(2)} X_{k}^{n}\right)^{2}}{\left(\Delta t_{k}^{n}\right)^{1 / 2+H}\left(\Delta t_{k+1}^{n}\right)^{1 / 2+H}\left(\Delta t_{k}^{n}+\Delta t_{k+1}^{n}\right)},
$$


where

$$
\Delta_{i r}^{(2)} X_{k}^{n}=\Delta t_{k}^{n} X\left(t_{k+1}^{n}\right)+\Delta t_{k+1}^{n} X\left(t_{k-1}^{n}\right)-\left(\Delta t_{k}^{n}+\Delta t_{k+1}^{n}\right) X\left(t_{k}^{n}\right) .
$$

\subsection{Auxiliary results}

Let $\mathcal{W}_{p}([a, b])$ denote the set of functions which have bounded $p$-variation on the interval $[a, b]$ :

$$
\mathcal{W}_{p}([a, b]):=\left\{f:[a, b] \rightarrow \mathbb{R}: v_{p}(f ;[a, b])<\infty\right\},
$$

where

$$
v_{p}(f ;[a, b])=\sup _{\varkappa} \sum_{k=1}^{n}\left|f\left(x_{k}\right)-f\left(x_{k-1}\right)\right|^{p},
$$

$\varkappa=\left\{x_{i}: i=0, \ldots, n\right\}$ being all finite partitions of $[a, b]$ such that $a=$ $x_{0}<x_{i}<\ldots<x_{n}=b$. Let $V_{p}(f):=V_{p}(f ;[a, b])=v_{p}^{1 / p}(f ;[a, b]) . V_{p}(f)$ is a non-increasing function of $p$, that is, if $0<q<p$ then $V_{p}(f) \leqslant V_{q}(f)$. Let $a<c<b$ and let $f \in \mathcal{W}_{p}([a, b])$ with $0<p<\infty$. Then

$$
\begin{aligned}
& v_{p}(f ;[a, c])+v_{p}(f ;[c, b]) \leqslant v_{p}(f ;[a, b]), \\
& V_{p}(f ;[a, b]) \leqslant V_{p}(f ;[a, c])+V_{p}(f ;[c, b]) .
\end{aligned}
$$

Let $f \in \mathcal{W}_{q}([a, b])$ and $h \in \mathcal{W}_{p}([a, b])$. The Love-Young inequality states that

$$
\left|\int_{a}^{b} f d h-f(y)[h(b)-h(a)]\right| \leqslant C_{p, q} V_{q}(f ;[a, b]) V_{p}(h ;[a, b])
$$

and

$$
V_{p}\left(\int_{a}^{\cdot} f d h ;[a, b]\right) \leqslant C_{p, q} V_{q, \infty}(f ;[a, b]) V_{p}(h ;[a, b]),
$$

where $V_{q, \infty}(f ;[a, b])=V_{q}(f ;[a, b])+\sup _{a \leqslant x \leqslant b}|f(x)|, C_{p, q}=\zeta\left(p^{-1}+q^{-1}\right)$ and $\zeta(s)=\sum_{n \geqslant 1} n^{-s}$. Let $f \in \mathcal{W}_{q}([a, b])$ and $g \in \mathcal{W}_{p}([a, b]), 0<p<\infty$. Then $f g \in \mathcal{W}_{p}([a, b])$ and

$$
V_{p, \infty}(f g ;[a, b]) \leqslant C_{p} V_{p, \infty}(f ;[a, b]) V_{p, \infty}(g ;[a, b]) .
$$


Let $f \in \mathcal{W}_{q}([a, b])$ and $g \in \mathcal{W}_{p}([a, b])$. For any partition $\varkappa$ and for $p^{-1}+q^{-1} \geqslant$ 1 the Young's version of Hölder's inequality and the inequality (1.2) yield

$$
\sum_{i} V_{q}\left(f ;\left[x_{i-1}, x_{i}\right]\right) V_{p}\left(g ;\left[x_{i-1}, x_{i}\right]\right) \leqslant V_{q}(f ;[a, b]) V_{p}(g ;[a, b]),
$$

since

$$
\begin{aligned}
& \sum_{i} V_{q}\left(f ;\left[x_{i-1}, x_{i}\right]\right) V_{p}\left(g ;\left[x_{i-1}, x_{i}\right]\right) \\
& \leqslant\left(\sum_{i} V_{q}^{q}\left(f ;\left[x_{i-1}, x_{i}\right]\right)\right)^{\frac{1}{q}} \cdot\left(\sum_{i} V_{p}^{p}\left(g ;\left[x_{i-1}, x_{i}\right]\right)\right)^{\frac{1}{p}} \\
& \quad=\left(\sum_{i} v_{q}\left(f ;\left[x_{i-1}, x_{i}\right]\right)\right)^{\frac{1}{q}} \cdot\left(\sum_{i} v_{p}\left(g ;\left[x_{i-1}, x_{i}\right]\right)\right)^{\frac{1}{p}} \\
& \leqslant\left(v_{q}(f ;[a, b])\right)^{\frac{1}{q}} \cdot\left(v_{p}(g ;[a, b])\right)^{\frac{1}{p}}=V_{q}(f ;[a, b]) V_{p}(g ;[a, b]) .
\end{aligned}
$$

Since almost all sample paths of the $B^{H}, 1 / 2 \leqslant H<1$, are locally Hölder continuous, it follows that

$$
V_{p}\left(B^{H} ;[s, t]\right) \leqslant L_{T}^{H, 1 / p}(t-s)^{1 / p},
$$

where $s<t \leqslant T, p>1 / H$,

$$
L_{T}^{H, \gamma}=\sup _{\substack{s \neq t \\ s, t \leqslant T}} \frac{\left|B_{t}^{H}-B_{s}^{H}\right|}{|t-s|^{\gamma}}, \quad 0<\gamma<H, \quad \mathbf{E}\left(L_{T}^{H, \gamma}\right)^{k}<\infty, \quad \forall k \geqslant 1 .
$$

\subsection{Conclusions of the first chapter}

The estimation of the Hurst index $H$ has been thoroughly studied for various types of Gaussian processes. The goal of this dissertation is to address such estimation when the underlying process is the solution of the stochastic differential equation (1.1) which is not necessarily Gaussian. 



\section{Quadratic variations and the increment ratios statistic}

\subsection{Regular subdivisions}

2.1 Theorem. Let $f$ be a Lipschitz-continuos function and let $g \in \mathcal{C}^{1+\alpha}, \frac{1}{H}-$ $1<\alpha \leqslant 1$. Assume that the subdivision of the interval $[0, T]$ is regular. Then

$$
\lim _{n \rightarrow \infty} n^{2 H-1} V_{n}^{(1)}(X, 2)=\int_{0}^{T} g^{2}\left(X_{t}\right) d t
$$

where $X$ is the solution of the equation (1.1).

Define

$$
\widehat{H}_{d v 1}^{n}:=\frac{1}{2}-\frac{1}{2 \ln 2} \ln \frac{V_{2 n}^{(1)}(X, 2)}{V_{n}^{(1)}(X, 2)} .
$$

Here and further $V_{2 n}^{(\cdot)}(X, 2)$ corresponds to the quadratic variation of the whole sample path while $V_{n}^{(\cdot)}(X, 2)$ is the variation of the subset $\left\{X_{k}: k=2 j, 0 \leqslant\right.$ $j \leqslant[n / 2]\},[x]$ denotes the integer part of $x$.

2.2 Theorem. Assume that conditions of Theorem 2.1 are satisfied. Then

$$
\widehat{H}_{d v 1}^{n} \longrightarrow H \quad \text { a.s. } \quad \text { as } n \rightarrow \infty .
$$


2.3 Theorem. Let $f$ be a Lipschitz-continuos function and let $g \in \mathcal{C}^{1+\alpha}, \frac{1}{H}-$ $1<\alpha \leqslant 1$. Assume that the subdivision of the interval $[0, T]$ is regular. Then

$$
\lim _{n \rightarrow \infty} n^{2 H-1} V_{n}^{(2)}(X, 2)=\left(4-2^{2 H}\right) \int_{0}^{T} g^{2}\left(X_{t}\right) d t
$$

where $X$ is the solution of (1.1).

Define

$$
\widehat{H}_{d v 2}^{n}:=\frac{1}{2}-\frac{1}{2 \ln 2} \ln \frac{V_{2 n}^{(2)}(X, 2)}{V_{n}^{(2)}(X, 2)} .
$$

2.4 Theorem. Assume that conditions of Theorem 2.3 are satisfied. Then

$$
\widehat{H}_{d v 2}^{n} \longrightarrow H \quad \text { a.s. } \quad \text { as } n \rightarrow \infty .
$$

The proof of Theorem 2.1 does not differ significantly from that of Theorem 2.6 and shall be omitted. The proof of Theorem 2.2 follows immediately from the proof of Theorem 2.4 and the result of Theorem 2.1.

Proof of Theorem 2.3. Under the conditions of the theorem the solution of the equation (1.1) exists for $1 \leqslant p<1+\alpha$ and $V_{p}(X ;[0, T])<\infty$ for every $p>1 / H$. For simplicity the index $n$ for $t$ in the sequel will be omitted. So

$$
\begin{aligned}
V_{n}^{2}(X, 2)= & \sum_{k=1}^{n-1}\left(\int_{t_{k}}^{t_{k+1}} f\left(X_{s}\right) d s-\int_{t_{k-1}}^{t_{k}} f\left(X_{s}\right) d s\right)^{2} \\
& +\sum_{k=1}^{n-1}\left(\int_{t_{k}}^{t_{k+1}} g\left(X_{s}\right) d B_{s}^{H}-\int_{t_{k-1}}^{t_{k}} g\left(X_{s}\right) d B_{s}^{H}\right)^{2} \\
+ & 2 \sum_{k=1}^{n-1}\left(\int_{t_{k}}^{t_{k+1}} g\left(X_{s}\right) d B_{s}^{H}-\int_{t_{k-1}}^{t_{k}} g\left(X_{s}\right) d B_{s}^{H}\right) \\
& \times\left(\int_{t_{k}}^{t_{k+1}} f\left(X_{s}\right) d s-\int_{t_{k-1}}^{t_{k}} f\left(X_{s}\right) d s\right) \\
=: & S_{1}+S_{2}+S_{12} .
\end{aligned}
$$


Denote $X_{k}:=X\left(t_{k}^{n}\right)$. Let's evaluate the behavior of

$$
\begin{aligned}
V_{n}^{(2)}(X, 2)-\sum_{k=1}^{n-1} g^{2}\left(X_{k}\right)\left(\Delta^{(2)} B_{k}^{H}\right)^{2} \\
\quad=S_{1}+\left(S_{2}-\sum_{k=1}^{n-1} g^{2}\left(X_{k}\right)\left(\Delta^{(2)} B_{k}^{H}\right)^{2}\right)+S_{12} \\
=: S_{1}+\widetilde{S_{2}}+S_{12} .
\end{aligned}
$$

Obviously,

$$
\begin{array}{rl}
\int_{t_{k}}^{t_{k+1}} & g\left(X_{s}\right) d B_{s}^{H}-\int_{t_{k-1}}^{t_{k}} g\left(X_{s}\right) d B_{s}^{H} \\
= & \left(\int_{t_{k}}^{t_{k+1}} g\left(X_{s}\right) d B_{s}^{H}-g\left(X_{k}\right) \Delta B_{k+1}^{H}\right) \\
& -\left(\int_{t_{k-1}}^{t_{k}} g\left(X_{s}\right) d B_{s}^{H}-g\left(X_{k}\right) \Delta B_{k}^{H}\right)+g\left(X_{k}\right) \Delta^{(2)} B_{k}^{H} .
\end{array}
$$

Thus

$$
\begin{aligned}
\left|\widetilde{S_{2}}\right| \leqslant & \sum_{k=1}^{n-1}\left(\int_{t_{k}}^{t_{k+1}} g\left(X_{s}\right) d B_{s}^{H}-g\left(X_{k}\right) \Delta B_{k+1}^{H}\right)^{2} \\
& +\sum_{k=1}^{n-1}\left(\int_{t_{k-1}}^{t_{k}} g\left(X_{s}\right) d B_{s}^{H}-g\left(X_{k}\right) \Delta B_{k}^{H}\right)^{2} \\
& +2 \sum_{k=1}^{n-1}\left|g\left(X_{k}\right) \Delta^{(2)} B_{k}^{H}\right| \cdot\left|\int_{t_{k}}^{t_{k+1}} g\left(X_{s}\right) d B_{s}^{H}-g\left(X_{k}\right) \Delta B_{k+1}^{H}\right| \\
& +2 \sum_{k=1}^{n-1}\left|g\left(X_{k}\right) \Delta^{(2)} B_{k}^{H}\right| \cdot\left|\int_{t_{k-1}}^{t_{k}} g\left(X_{s}\right) d B_{s}^{H}-g\left(X_{k}\right) \Delta B_{k}^{H}\right| .
\end{aligned}
$$

Further, from the Love-Young inequality (1.4) it follows that

$$
\begin{aligned}
\left|\widetilde{S_{2}}\right| & \leqslant 2 C_{p, p}^{2}\left|g^{\prime}\right|_{\infty}^{2} \sum_{k=0}^{n-1} V_{p}^{2}\left(X ;\left[t_{k}, t_{k+1}\right]\right) V_{p}^{2}\left(B^{H} ;\left[t_{k}, t_{k+1}\right]\right) \\
& +2 C_{p, p}\left|g^{\prime}\right|_{\infty} \sum_{k=1}^{n-1}\left|g\left(X_{k}\right) \Delta^{(2)} B_{k}^{H}\right| \cdot V_{p}\left(X ;\left[t_{k}, t_{k+1}\right]\right) V_{p}\left(B^{H} ;\left[t_{k}, t_{k+1}\right]\right)
\end{aligned}
$$




$$
+2 C_{p, p}\left|g^{\prime}\right|_{\infty} \sum_{k=1}^{n-1}\left|g\left(X_{k}\right) \Delta^{(2)} B_{k}^{H}\right| \cdot V_{p}\left(X ;\left[t_{k-1}, t_{k}\right]\right) V_{p}\left(B^{H} ;\left[t_{k-1}, t_{k}\right]\right)
$$

which, coupled with the inequality (1.7), yields

$$
\begin{aligned}
\left|\widetilde{S_{2}}\right| \leqslant & 2 C_{p, p}^{2}\left|g^{\prime}\right|_{\infty}^{2} \max _{0 \leqslant k \leqslant n-1}\left[V_{p}\left(X ;\left[t_{k}, t_{k+1}\right]\right) V_{p}\left(B^{H} ;\left[t_{k}, t_{k+1}\right]\right)\right] \\
& \times V_{p}(X ;[0, T]) V_{p}\left(B^{H} ;[0, T]\right) \\
& +4 C_{p, p}\left|g^{\prime}\right|_{\infty} \max _{1 \leqslant k \leqslant n-1}\left|g\left(X_{k}\right) \Delta^{(2)} B_{k}^{H}\right| V_{p}(X ;[0, T]) V_{p}\left(B^{H} ;[0, T]\right) \\
\leqslant & 2 C_{p, p}^{2}\left|g^{\prime}\right|_{\infty}^{2} \max _{0 \leqslant k \leqslant n-1}\left[V_{p}\left(B^{H} ;\left[t_{k}, t_{k+1}\right]\right)\right] V_{p}^{2}(X ;[0, T]) V_{p}\left(B^{H} ;[0, T]\right) \\
+ & 8 C_{p, p}\left|g^{\prime}\right|_{\infty} \max _{1 \leqslant k \leqslant n}\left|\Delta B_{k}^{H}\right|\left[\left|g^{\prime}\right|_{\infty} V_{p}(X ;[0, T])+|g(\xi)|\right] \\
& \times V_{p}(X ;[0, T]) V_{p}\left(B^{H} ;[0, T]\right)
\end{aligned}
$$

since for all $0 \leqslant k \leqslant n$

$$
\left|g\left(X_{k}\right)\right| \leqslant\left|g^{\prime}\right|_{\infty} V_{p}(X ;[0, T])+|g(\xi)| .
$$

$\left|S_{12}\right|$ can be rewritten as

$$
\begin{aligned}
\left|S_{12}\right|= & 2 \sum_{k=1}^{n-1} \mid \int_{t_{k}}^{t_{k+1}} g\left(X_{s}\right) d B_{s}^{H}-g\left(X_{k}\right) \Delta B_{k}^{H} \\
& -\int_{t_{k-1}}^{t_{k}} g\left(X_{s}\right) d B_{s}^{H}+g\left(X_{k}\right) \Delta B_{k}^{H} \mid \\
& \times\left|\int_{t_{k}}^{t_{k+1}} f\left(X_{s}\right) d s-\int_{t_{k-1}}^{t_{k}} f\left(X_{s}\right) d s\right| .
\end{aligned}
$$

From the Love-Young inequality (1.4) it follows that

$$
\begin{aligned}
\left|S_{12}\right| \leqslant & 2 C_{p, p} \sum_{k=1}^{n-1}\left\{\left[V_{p}\left(g(X) ;\left[t_{k}, t_{k+1}\right]\right) V_{p}\left(B^{H} ;\left[t_{k}, t_{k+1}\right]\right)\right.\right. \\
& \left.+V_{p}\left(g(X) ;\left[t_{k-1}, t_{k}\right]\right) V_{p}\left(B^{H} ;\left[t_{k-1}, t_{k}\right]\right)\right] \\
& \left.\times\left[\int_{t_{k}}^{t_{k+1}}\left|f\left(X_{s}\right)\right| d s+\int_{t_{k-1}}^{t_{k}}\left|f\left(X_{s}\right)\right| d s\right]\right\} \\
\leqslant & 4 C_{p, p} \max _{1 \leqslant k \leqslant n}\left[V_{p}\left(g(X) ;\left[t_{k-1}, t_{k}\right]\right) V_{p}\left(B^{H} ;\left[t_{k-1}, t_{k}\right]\right)\right]
\end{aligned}
$$




$$
\begin{aligned}
& \quad \times \sum_{k=1}^{n-1}\left[\int_{t_{k}}^{t_{k+1}}\left|f\left(X_{s}\right)\right| d s+\int_{t_{k-1}}^{t_{k}}\left|f\left(X_{s}\right)\right| d s\right] \\
& \leqslant 8 C_{p, p}\left|g^{\prime}\right|_{\infty} V_{p}(X ;[0, T]) \max _{1 \leqslant k \leqslant n}\left[V_{p}\left(B^{H} ;\left[t_{k-1}, t_{k}\right]\right)\right] \\
& \quad \times \int_{0}^{T}\left|f\left(X_{s}\right)\right| d s .
\end{aligned}
$$

Additionally,

$$
\left|f\left(X_{s}\right)\right| \leqslant\left|f\left(X_{s}\right)-f(\xi)\right|+|f(\xi)| \leqslant L V_{p}(X ;[0, T])+|f(\xi)|,
$$

where $L$ is the Lipschitz constant of the function $f$. Therefore,

$$
\begin{aligned}
\left|S_{12}\right| \leqslant & 8 C_{p, p}\left|g^{\prime}\right|_{\infty} V_{p}(X ;[0, T]) \max _{1 \leqslant k \leqslant n}\left[V_{p}\left(B^{H} ;\left[t_{k-1}, t_{k}\right]\right)\right] \\
& \times\left[L V_{p}(X ;[0, T])+|f(\xi)|\right] \text { and } \\
S_{1}= & \sum_{k=1}^{n-1}\left(\int_{t_{k}}^{t_{k+1}} f\left(X_{s}\right) d s-\int_{t_{k-1}}^{t_{k}} f\left(X_{s}\right) d s\right)^{2} \\
\leqslant & 2 \sum_{k=0}^{n-1}\left(\int_{t_{k}}^{t_{k+1}} f\left(X_{s}\right) d s\right)^{2} \leqslant 2 n^{-1} \int_{0}^{T} f^{2}\left(X_{s}\right) d s \\
\leqslant & 2 T n^{-1}\left[|f(\xi)|+L V_{p}(X ;[0, T])\right]^{2} .
\end{aligned}
$$

Note that for $p$ such that $H-1 / p<1-H$ it follows that

$$
\begin{aligned}
& n^{2 H-1} \max \left\{\left|\Delta B_{k}^{H}\right|, V_{p}\left(B^{H} ;\left[t_{k}, t_{k+1}\right]\right)\right\} \\
& \quad \leqslant L_{T}^{H, 1 / p} T^{1 / p} n^{2 H-1-1 / p} \stackrel{\text { a.s. }}{\longrightarrow} 0 \text { as } n \rightarrow \infty .
\end{aligned}
$$

Such a value of $p$ will always exist. Therefore it follows that

$$
n^{2 H-1}\left|S_{1}+\tilde{S}_{2}+S_{12}\right| \stackrel{\text { a.s. }}{\longrightarrow} 0 \quad \text { as } n \rightarrow \infty .
$$

Consequently, the theorem will be proved if the convergence

$$
n^{2 H-1} \sum_{k=1}^{n-1} g^{2}\left(X_{k}\right)\left(\Delta^{(2)} B_{k}^{H}\right)^{2} \stackrel{\text { a.s. }}{\longrightarrow}\left(4-2^{2 H}\right) \int_{0}^{T} g^{2}\left(X_{t}\right) d t
$$

is obtained. To do so, the Helly-Bray theorem is applied. 
2.5 Theorem. Let the functions $F_{n}(n=1,2, \ldots)$ be non-decreasing and uniformly bounded. If the sequence $F_{n}$ converges to $F$ in its continuity points and

$$
F_{n}(-\infty) \rightarrow F(-\infty), \quad F_{n}(\infty) \rightarrow F(\infty),
$$

then for every continuous bounded function $g$

$$
\int_{-\infty}^{\infty} g(x) d F_{n}(x) \rightarrow \int_{-\infty}^{\infty} g(x) d F(x)
$$

Let

$$
V_{n}^{(2)}\left(B^{H}, 2\right)_{t}=\sum_{k=1}^{[n t / T]-1}\left(\Delta^{(2)} B_{k}^{H}\right)^{2}, \quad t \in[0, T]
$$

and $S_{n}(t)=n^{2 H-1} V_{n}^{(2)}\left(B^{H}, 2\right)(t)$. Then

$$
n^{2 H-1} \sum_{k=1}^{n-1} g^{2}\left(X_{k}\right)\left(\Delta^{(2)} B_{k}^{H}\right)^{2}=\int_{0}^{T} g^{2}\left(X_{t}\right) d S_{n}(t) .
$$

It is known (see, e.g., Bégyn (2006) 122p.) that

$$
n^{2 H-1} V_{n}^{2}\left(B^{H}, 2\right)_{t} \stackrel{\text { a.s. }}{\longrightarrow}\left(4-2^{2 H}\right) t .
$$

Since the function $S_{t}^{n}$ is non-decreasing, it follows that (see Lemma 1 in McLeish (1978))

$$
\sup _{t \leqslant T}\left|S_{t}^{n}-t\right| \stackrel{\text { a.s. }}{\longrightarrow} 0 \quad \text { as } n \rightarrow \infty .
$$

The function $S_{t}^{n}$ is non-decreasing and uniformly bounded for every $\omega$. Therefore the Helly-Bray theorem yields

$$
\int_{0}^{T} g^{2}\left(X_{t}\right) d S_{n}(t) \stackrel{\text { a.s. }}{\longrightarrow}\left(4-2^{2 H}\right) \int_{0}^{T} g^{2}\left(X_{t}\right) d t \quad \text { as } \quad n \rightarrow \infty
$$

which completes the proof.

Proof of Theorem 2.4. The estimator $\widehat{H}_{d v 2}^{n}$ can be rewritten as

$$
\begin{aligned}
\widehat{H}_{d v 2}^{n}=\frac{1}{2} & -\frac{1}{2 \ln 2}\left[(2 H-1) \ln \frac{1}{2}\right. \\
& \left.+\ln \frac{(2 n)^{2 H-1} V_{2 n}(X, 2)}{(n)^{2 H-1} V_{n}(X, 2)}\right]
\end{aligned}
$$




$$
=H-\frac{1}{2 \ln 2} \ln \frac{(2 n)^{2 H-1} V_{2 n}(X, 2)}{(n)^{2 H-1} V_{n}(X, 2)},
$$

which, coupled with the results of Theorem 2.3 yields the convergence

$$
\widehat{H}_{d v 2}^{n}-H \stackrel{\text { a.s. }}{\longrightarrow} 0 \text {. }
$$

\subsection{Irregular subdivisions}

2.6 Theorem. Let $f$ be a Lipschitz-continuos function and let $g \in \mathcal{C}^{1+\alpha}, \frac{1}{H}-$ $1<\alpha \leqslant 1$. Let $\left(\pi_{n}\right)_{n \in \mathbb{N}}$ be a sequence of subdivisions of the interval $[0, T]$ such that

$$
m_{n}^{2-2 H} \stackrel{\mathbf{n} \rightarrow \infty}{=} o(1 / \ln n) \quad \text { and } \quad m_{n} \stackrel{\mathbf{n} \rightarrow \infty}{=} \mathcal{O}\left(p_{n}\right) .
$$

Then

$$
V_{\pi_{n}}^{(1)}(X, 2) \stackrel{\text { a.s. }}{\longrightarrow} \int_{0}^{T} g^{2}\left(X_{t}\right) d t \quad \text { as } m_{n} \rightarrow 0,
$$

where $X$ is the solution of (1.1).

Let $\left(\pi_{n}\right)_{n \geqslant 1}$ be a sequence of partitions of $[0, T]$ such that $0=t_{0}^{n}<$ $t_{1}^{n}<\cdots<t_{N_{n}}^{n}=T$ for all $n \geqslant 1$. Assume that we have two sequences of partitions $\left(\pi_{i(n)}\right)_{n \geqslant 1}$ and $\left(\pi_{j(n)}\right)_{n \geqslant 1}$ of $[0, T]$ such that $\pi_{i(n)} \subset \pi_{j(n)} \subseteq \pi_{n}$, $i(n)<j(n) \leqslant N_{n}$, for all $n \in \mathbb{N}$, where $\pi_{i(n)}=\left\{0=t_{0}^{n}<t_{i(1)}^{n}<t_{i(2)}^{n}<\right.$ $\left.\cdots<t_{i(n)}^{n}=T\right\}$ and $\pi_{j(n)}=\left\{0=t_{0}^{n}<t_{j(1)}^{n}<t_{j(2)}^{n}<\cdots<t_{j(n)}^{n}=T\right\}$.

Define

$\widetilde{H}_{d v 1}^{n}:=\frac{1}{2}-\frac{1}{2 \ln \left(m_{i(n)} / p_{j(n)}\right)} \ln \frac{V_{j(n)}^{(1)}(X, 2)}{V_{i(n)}^{(1)}(X, 2)}, \quad V_{i(n)}^{(1)}(X, 2)=\sum_{k=1}^{i(n)}\left(\Delta X_{k}^{n}\right)^{2}$,

where

$\Delta t_{k}^{n}=t_{i(k)}^{n}-t_{i(k-1)}^{n}, \quad m_{i(n)}=\max _{1 \leqslant k \leqslant i(n)} \Delta t_{k}^{n}, \quad p_{i(n)}=\min _{1 \leqslant k \leqslant i(n)} \Delta t_{k}^{n}$.

2.7 Theorem. Assume that conditions of Theorem 2.6 are satisfied. If the sequences of partitions $\left(\pi_{i(n)}\right)$ and $\left(\pi_{j(n)}\right), i(n)<j(n)$, are regular or such that $\ln \left(p_{i(n)} / p_{j(n)}\right) \rightarrow \infty$ as $n \rightarrow \infty$, then

$$
\widetilde{H}_{d v 1}^{n} \longrightarrow H \quad \text { a.s. } \quad \text { as } n \rightarrow \infty .
$$


To study the almost sure convergence of the second order quadratic variations of $X$ additional assumptions on the sequence $\left(\pi_{n}\right)_{n \in \mathbb{N}}$ are required.

2.1 Definition. (see Bégyn (2006)) Let $\left(\ell_{k}\right)_{k \geqslant 1}$ be a sequence of real numbers in the interval $(0, \infty)$. We say that $\left(\pi_{n}\right)_{n \in \mathbb{N}}$ is a sequence of subdivisions with asymptotic ratios $\left(\ell_{k}\right)_{k \geqslant 1}$ if it satisfies the following assumptions:

- $m_{n} \stackrel{\mathbf{n} \rightarrow \infty}{=} \mathcal{O}\left(p_{n}\right)$;

- $\lim _{n \rightarrow \infty} \sup _{1 \leqslant k \leqslant N_{n}}\left|\frac{\Delta t_{k-1}^{n}}{\Delta t_{k}^{n}}-\ell_{k}\right|=0$.

The set $\mathcal{L}=\left\{\ell_{1}, \ell_{2}, \ldots, \ell_{k}, \ldots\right\}$ will be called the range of the asymptotic ratios of the sequence $\left(\pi_{n}\right)_{n \in \mathbb{N}}$.

It is clear that if the sequence $\left(\pi_{n}\right)_{n \in \mathbb{N}}$ is regular, then it is a sequence with asymptotic ratios $\ell_{k}=1$ for all $k \geqslant 1$.

2.2 Definition. (see Bégyn (2006)) The function $g:(0, \infty) \rightarrow \mathbb{R}$ is invariant on $\mathcal{L}$ if for all $\ell, \hat{\ell} \in \mathcal{L}, g(\ell)=g(\hat{\ell})$.

For example, let $\mathcal{L}=\left\{\alpha, \alpha^{-1}\right\}$ be the set containing two real positive numbers and let

$$
h(\lambda)=\frac{1+\lambda^{2 H-1}-(1+\lambda)^{2 H-1}}{\lambda^{H-1 / 2}} .
$$

The function $h$ is invariant on $\mathcal{L}$.

2.8 Theorem. Let $f$ be a Lipschitz-continuos function and let $g \in \mathcal{C}^{1+\alpha}, \frac{1}{H}-$ $1<\alpha \leqslant 1$. Let $\left(\pi_{n}\right)_{n \in \mathbb{N}}$ be a sequence of subdivisions with asymptotic ratios $\left(\ell_{k}\right)_{k \geqslant 1}$ and range of the asymptotic ratios $\mathcal{L}$. Assume that the lower mesh of the subdivisions $\pi_{n}$ satisfy $p_{n} \stackrel{\mathbf{n} \rightarrow \infty}{=} o(1 / \ln n)$ and

$$
h(\lambda)=\frac{1+\lambda^{2 H-1}-(1+\lambda)^{2 H-1}}{\lambda^{H-1 / 2}} .
$$

Let $X$ be the solution of (1.1). If the function $h$ is invariant on $\mathcal{L}$ or the sequence of functions $\ell_{n}(t)$ converges uniformly to $\ell(t)$ on the interval $[0, T]$, where

$$
\ell_{n}(t)=\sum_{k=1}^{N_{n}-1} \ell_{k} \mathbf{1}_{\left[t_{k}^{n}, t_{k+1}^{n}\right)}(t),
$$


then

$$
\lim _{n \rightarrow \infty} V_{\pi_{n}}^{(2)}(X, 2)=2 \int_{0}^{T} g^{2}\left(X_{t}\right) h(\ell(t)) d t
$$

Proof of Theorem 2.6. The $p$-variation boundedness of almost all paths of solution of the equation (1.1) implies the existence of the integrals $\int_{0}^{T} f^{2}\left(X_{t}\right) d t$ and $\int_{0}^{T} g^{2}\left(X_{t}\right) d t$

Set $X_{k}^{n}:=X\left(t_{k}^{n}\right)$. For simplicity, the index $H$ for $B$ will be omitted in the sequel. Note that

$$
\begin{aligned}
\left(\Delta X_{k}^{n}\right)^{2}= & \left(\int_{t_{k-1}^{n}}^{t_{k}^{n}} f\left(X_{s}\right) d s\right)^{2}+2 \int_{t_{k-1}^{n}}^{t_{k}^{n}} f\left(X_{s}\right) d s \cdot \int_{t_{k}^{n}}^{t_{k}^{n}} g\left(X_{s}\right) d B_{s} \\
& +\left(\int_{t_{k-1}^{n}}^{t_{k}^{n}}\left[g\left(X_{s}\right)-g\left(X_{k}^{n}\right)\right] d B_{s}\right)^{2}+g^{2}\left(X_{k}^{n}\right)\left(\Delta B_{k}^{n}\right)^{2} \\
& +2 g\left(X_{k}^{n}\right) \Delta B_{k}^{n}\left(\int_{t_{k-1}^{n}}^{t_{k}^{n}}\left[g\left(X_{s}\right)-g\left(X_{k}^{n}\right)\right] d B_{s}\right) \\
= & \sum_{j=1}^{5} I_{n, k}^{(j)} .
\end{aligned}
$$

It'll be proved that

$$
\sum_{\substack{j=1 \\ j \neq 4}}^{5} I_{n, k}^{(j)} \stackrel{\text { a.s. }}{\longrightarrow} 0 \quad \text { as } \quad m_{n} \rightarrow 0
$$

Note that

$$
\left|I_{n, k}^{(1)}\right| \leqslant \Delta t_{k}^{n} \int_{t_{k-1}^{n}}^{t_{k}^{n}} f^{2}\left(X_{s}\right) d s .
$$

By Love-Young inequality (1.4) for all $p>1 / H$

$$
\begin{aligned}
\left|I_{n, k}^{(2)}\right| \leqslant & 2\left|\int_{t_{k-1}^{n}}^{t_{k}^{n}} f\left(X_{s}\right) d s\right| \cdot\left|g\left(X_{k}\right) \Delta B_{k}^{n}\right| \\
& +2\left|\int_{t_{k-1}^{n}}^{t_{k}^{n}} f\left(X_{s}\right) d s\right| \cdot\left|\int_{t_{k-1}^{n}}^{t_{k}^{n}}\left[g\left(X_{s}\right)-g\left(X_{k}^{n}\right)\right] d B_{s}\right| \\
\leqslant & 2\left|\Delta B_{k}^{n}\right| \sup _{t \leqslant T}\left|g\left(X_{t}\right)\right| \int_{t_{k-1}^{n}}^{t_{k}^{n}}\left|f\left(X_{s}\right)\right| d s
\end{aligned}
$$




$$
\begin{aligned}
& +2 C_{p, p}\left|g^{\prime}\right|_{\infty} V_{p}\left(X ;\left[t_{k}^{n} ; t_{k+1}^{n}\right]\right) V_{p}\left(B ;\left[t_{k}^{n} ; t_{k+1}^{n}\right]\right) \int_{t_{k-1}^{n}}^{t_{k}^{n}}\left|f\left(X_{s}\right)\right| d s, \\
\left|I_{n, k}^{(3)}\right| \leqslant & C_{p, p}^{2}\left|g^{\prime}\right|_{\infty}^{2} V_{p}^{2}\left(X ;\left[t_{k}^{n} ; t_{k+1}^{n}\right]\right) V_{p}^{2}\left(B ;\left[t_{k}^{n} ; t_{k+1}^{n}\right]\right), \\
\left|I_{n, k}^{(5)}\right| \leqslant & 2 C_{p, p}\left|g^{\prime}\right|_{\infty}\left|\Delta B_{k}^{n}\right| \sup _{t \leqslant T}\left|g\left(X_{t}\right)\right| V_{p}\left(X ;\left[t_{k}^{n} ; t_{k+1}^{n}\right]\right) V_{p}\left(B ;\left[t_{k}^{n} ; t_{k+1}^{n}\right]\right) .
\end{aligned}
$$

It is evident that

$$
\begin{aligned}
& \sum_{k=1}^{N_{n}}\left(\Delta t_{k}\right)^{1-2 H}\left|I_{n, k}^{(1)}\right| \leqslant m_{n}^{2-2 H} \int_{0}^{T} f^{2}\left(X_{s}\right) d s \\
& \sum_{k=1}^{N_{n}}\left(\Delta t_{k}\right)^{1-2 H}\left|I_{n, k}^{(2)}\right| \leqslant 2 \max _{1 \leqslant k \leqslant N_{n}} \frac{\left|\Delta B_{k}^{n}\right|}{\left(\Delta t_{k}\right)^{2 H-1}} \sup _{t \leqslant T}\left|g\left(X_{t}\right)\right| \int_{0}^{T}\left|f\left(X_{s}\right)\right| d s \\
& \quad+2 C_{p, p}\left|g^{\prime}\right|_{\infty} \max _{1 \leqslant k \leqslant N_{n}-1} \frac{V_{p}\left(B ;\left[t_{k}, t_{k+1}\right]\right)}{\left(\Delta t_{k}\right)^{2 H-1}} V_{p}(X ;[0, T]) \int_{0}^{T}\left|f\left(X_{s}\right)\right| d s .
\end{aligned}
$$

By using the inequality (1.7) the remaining two terms are estimated as

$$
\begin{aligned}
& \sum_{k=1}^{N_{n}}\left(\Delta t_{k}\right)^{1-2 H}\left|I_{n, k}^{(3)}\right| \\
& \quad \leqslant C_{p, p}^{2}\left|g^{\prime}\right|_{\infty}^{2} \max _{1 \leqslant k \leqslant N_{n}-1} \frac{V_{p}\left(B ;\left[t_{k}, t_{k+1}\right]\right)}{\left(\Delta t_{k}\right)^{2 H-1}} V_{p}^{2}(X ;[0, T]) V_{p}(B ;[0, T]), \\
& \sum_{k=1}^{N_{n}}\left(\Delta t_{k}\right)^{1-2 H}\left|I_{n, k}^{(5)}\right| \\
& \quad \leqslant 2 C_{p, p}\left|g^{\prime}\right|_{\infty} \max _{1 \leqslant k \leqslant N_{n}} \frac{\left|\Delta B_{k}^{n}\right|}{\left(\Delta t_{k}\right)^{2 H-1}} \sup _{t \leqslant T}\left|g\left(X_{t}\right)\right| V_{p}(X ;[0, T]) V_{p}(B ;[0, T]) .
\end{aligned}
$$

By (1.8) it follows that

$$
\max _{1 \leqslant k \leqslant N_{n}}\left|\Delta B_{k}^{n}\right| \leqslant L_{T}^{H, 1 / p} m_{n}^{1 / p}, \quad \max _{1 \leqslant k \leqslant N_{n}}\left[V_{p}\left(B ;\left[t_{k-1}^{n} ; t_{k}^{n}\right]\right)\right] \leqslant L_{T}^{H, 1 / p} m_{n}^{1 / p} .
$$

All the inequalities obtained above are correct for every $p>1 / H$. Thus there can always be chosen such a $p$ that $1 / p+1-2 H>0$. For this value of $p$

$$
V_{\pi_{n}}^{(1)}(X, 2)-\sum_{k=1}^{N_{n}} g^{2}\left(X_{k}\right) \frac{\left(\Delta B_{k}^{n}\right)^{2}}{\left(\Delta t_{k}^{n}\right)^{2 H-1}} \stackrel{\text { a.s. }}{\longrightarrow} 0
$$


as $m_{n} \rightarrow 0$. Consequently, the theorem will be proved if the convergence

$$
\sum_{k=1}^{N_{n}} g^{2}\left(X_{k}^{n}\right) \frac{\left(\Delta B_{k}^{n}\right)^{2}}{\left(\Delta t_{k}^{n}\right)^{2 H-1}} \stackrel{\text { a.s. }}{\longrightarrow} \int_{0}^{T} g^{2}\left(X_{t}\right) d t .
$$

is obtained. Set

$$
S_{t}^{n}=\sum_{k=1}^{r^{n}(t)} \frac{\left(\Delta B_{k}^{n}\right)^{2}}{\left(\Delta t_{k}^{n}\right)^{2 H-1}}, \quad t \in[0, T],
$$

where $r^{n}(t)=\max \left\{k: t_{k}^{n} \leqslant t\right\}$. Then

$$
\sum_{k=1}^{N_{n}} g^{2}\left(X_{k}^{n}\right) \frac{\left(\Delta B_{k}^{n}\right)^{2}}{\left(\Delta t_{k}^{n}\right)^{2 H-1}}=\int_{0}^{T} g^{2}\left(X_{t}\right) d S_{t}^{n}
$$

It is known (see Gine, Klein (1975)) that $S_{t}^{n} \stackrel{\text { a.s. }}{\longrightarrow} t$ if

$$
m_{n}^{2-2 H} \stackrel{\mathbf{n} \rightarrow \infty}{=} O(1 / \ln n) \quad \text { and } \quad m_{n} \stackrel{\mathbf{n} \rightarrow \infty}{=} \mathcal{O}\left(p_{n}\right) .
$$

The function $S_{t}^{n}$ is non-decreasing and uniformly bounded. Consequently, the Helly-Bray theorem implies that

$$
\int_{0}^{T} g^{2}\left(X_{t}\right) d S_{t}^{n} \stackrel{\text { a.s. }}{\longrightarrow} \int_{0}^{T} g^{2}\left(X_{t}\right) d t \quad \text { as } \quad n \rightarrow \infty .
$$

This completes the proof of the theorem.

Proof of Theorem 2.7. Note that

$$
\frac{m_{j(n)}^{1-2 H} V_{j(n)}^{(1)}(X, 2)}{p_{i(n)}^{1-2 H} V_{i(n)}^{(1)}(X, 2)} \leqslant \frac{V_{\pi_{j(n)}}^{(1)}(X, 2)}{V_{\pi_{i(n)}}^{(1)}(X, 2)} \leqslant \frac{p_{j(n)}^{1-2 H} V_{j(n)}^{(1)}(X, 2)}{m_{i(n)}^{1-2 H} V_{i(n)}^{(1)}(X, 2)} .
$$

It is evident that

$$
\begin{aligned}
\widehat{H}_{n}^{(1)}= & \frac{1}{2}-\frac{1}{2 \ln \left(m_{i(n)} / p_{j(n)}\right)} \\
& \times\left[(2 H-1) \ln \left(p_{j(n)} / m_{i(n)}\right)+\ln \frac{p_{j(n)}^{1-2 H} V_{j(n)}^{(1)}(X, 2)}{m_{i(n)}^{1-2 H} V_{i(n)}^{(1)}(X, 2)}\right]
\end{aligned}
$$




$$
\begin{aligned}
= & H-\frac{1}{2 \ln \left(m_{i(n)} / p_{j(n)}\right)} \ln \frac{p_{j(n)}^{1-2 H} V_{j(n)}^{(1)}(X, 2)}{m_{i(n)}^{1-2 H} V_{i(n)}^{(1)}(X, 2)} \\
= & H-\frac{1}{2 \ln \left(m_{i(n)} / p_{j(n)}\right)} \\
& \times\left[\ln \frac{V_{\pi_{j(n)}^{(1)}}^{(X, 2)}}{V_{\pi_{i(n)}^{(1)}}^{(1)}(X, 2)}+\ln \left(\frac{p_{j(n)}^{1-2 H} V_{j(n)}^{(1)}(X, 2)}{m_{i(n)}^{1-2 H} V_{i(n)}^{(1)}(X, 2)} / \frac{V_{\pi_{j(n)}}^{(1)}(X, 2)}{V_{\pi_{i(n)}}^{(1)}(X, 2)}\right)\right] .
\end{aligned}
$$

From the inequality (2.9) it follows that

$$
\ln \left(\frac{p_{j(n)}^{1-2 H} V_{j(n)}^{(1)}(X, 2)}{m_{i(n)}^{1-2 H} V_{i(n)}^{(1)}(X, 2)} / \frac{V_{\pi_{j(n)}}^{(1)}(X, 2)}{V_{\pi_{i(n)}}^{(1)}(X, 2)}\right) \geqslant 0 .
$$

Also $\ln \left(m_{i(n)} / p_{j(n)}\right)>0$. Thus

$$
\widehat{H}_{n}^{(1)} \leqslant H-\frac{1}{2 \ln \left(m_{i(n)} / p_{j(n)}\right)} \ln \frac{V_{\pi_{j(n)}}^{(1)}(X, 2)}{V_{\pi_{i(n)}}^{(1)}(X, 2)} .
$$

Further,

$$
\begin{aligned}
\widehat{H}_{n}^{(1)}= & \frac{1}{2}-\frac{1}{2 \ln \left(m_{i(n)} / p_{j(n)}\right)} \\
& \times\left[(2 H-1) \ln \left(m_{j(n)} / p_{i(n)}\right)+\ln \frac{m_{j(n)}^{1-2 H} V_{j(n)}^{(1)}(X, 2)}{p_{i(n)}^{1-2 H} V_{i(n)}^{(1)}(X, 2)}\right] \\
= & \frac{1}{2}-\left(H-\frac{1}{2}\right) \frac{\ln \left(m_{j(n)} / p_{i(n)}\right)}{\ln \left(m_{i(n)} / p_{j(n)}\right)} \\
& -\frac{1}{2 \ln \left(m_{i(n)} / p_{j(n)}\right)} \ln \frac{m_{j(n)}^{1-2 H} V_{j(n)}^{(1)}(X, 2)}{p_{i(n)}^{1-2 H} V_{i(n)}^{(1)}(X, 2)} \\
= & H+\left(H-\frac{1}{2}\right) \frac{\ln \left(p_{i(n)} / m_{j(n)}\right)-\ln \left(m_{i(n)} / p_{j(n)}\right)}{\ln \left(m_{i(n)} / p_{j(n)}\right)} \\
& -\frac{1}{2 \ln \left(m_{i(n)} / p_{j(n)}\right)}\left[\ln \frac{V_{\pi_{j(n)}}^{(1)}(X, 2)}{V_{\pi_{i(n)}^{(1)}}^{(1)}(X, 2)}\right.
\end{aligned}
$$




$$
\left.+\ln \left(\frac{m_{j(n)}^{1-2 H} V_{j(n)}^{(1)}(X, 2)}{p_{i(n)}^{1-2 H} V_{i(n)}^{(1)}(X, 2)} / \frac{V_{\pi_{j(n)}}^{(1)}(X, 2)}{V_{\pi_{i(n)}}^{(1)}(X, 2)}\right)\right] .
$$

Again, from (2.9) it follows that

$$
\ln \left(\frac{m_{j(n)}^{1-2 H} V_{j(n)}^{(1)}(X, 2)}{p_{i(n)}^{1-2 H} V_{i(n)}^{(1)}(X, 2)} / \frac{V_{\pi_{j(n)}}^{(1)}(X, 2)}{V_{\pi_{i(n)}}^{(1)}(X, 2)}\right) \leqslant 0
$$

which implies

$$
\begin{aligned}
\widehat{H}_{n}^{(1)} \geqslant & H+\left(H-\frac{1}{2}\right) \frac{\ln \left(p_{i(n)} / m_{i(n)}\right)+\ln \left(p_{j(n)} / m_{j(n)}\right)}{\ln \left(m_{i(n)} / p_{j(n)}\right)} \\
& -\frac{1}{2 \ln \left(m_{i(n)} / p_{j(n)}\right)} \ln \frac{V_{\pi_{j(n)}}^{(1)}(X, 2)}{V_{\pi_{i(n)}}^{(1)}(X, 2)} .
\end{aligned}
$$

If the sequences of partitions $\left(\pi_{i(n)}\right)$ and $\left(\pi_{j(n)}\right), i(n)<j(n)$, are regular then the second term in the inequality (2.2) is equal to 0 . If $\ln \left(p_{i(n)} / p_{j(n)}\right) \rightarrow$ $\infty, n \rightarrow \infty$, then the second term in the inequality (2.2) converges to 0 . By Theorem 2.6 we get that

$$
\frac{1}{2 \ln \left(m_{i(n)} / p_{j(n)}\right)} \ln \frac{V_{\pi_{j(n)}}^{(1)}(X, 2)}{V_{\pi_{i(n)}}^{(1)}(X, 2)} \longrightarrow 0 \quad \text { a.s. } \quad \text { as } n \rightarrow \infty .
$$

Therefore the convergence $\widehat{H}_{n}^{(1)} \longrightarrow H$ a.s. as $n \rightarrow \infty$ holds.

Proof of Theorem 2.8. It is obvious that the square of the second order increments can be rewritten as

$$
\begin{aligned}
&\left(\Delta_{i r}^{(2)} X_{k}^{n}\right)^{2}=\left(\Delta t_{k}^{n} \int_{t_{k}^{n}}^{t_{k+1}} f\left(X_{s}\right) d s-\Delta t_{k+1}^{n} \int_{t_{k-1}^{n}}^{t_{k}^{n}} f\left(X_{s}\right) d s\right)^{2} \\
&+2\left(\Delta t_{k}^{n} \int_{t_{k}^{n}}^{t_{k+1}^{n}}\left[g\left(X_{s}\right)-g\left(X_{k}^{n}\right)\right] d B_{s}\right. \\
&\left.-\Delta t_{k+1}^{n} \int_{t_{k-1}^{n}}^{t_{k}^{n}}\left[g\left(X_{s}\right)-g\left(X_{k}^{n}\right)\right] d B_{s}\right)
\end{aligned}
$$




$$
\begin{aligned}
& \times\left(\Delta t_{k}^{n} \int_{t_{k}^{n}}^{t_{k+1}^{n}} f\left(X_{s}\right) d s-\Delta t_{k+1}^{n} \int_{t_{k-1}^{n}}^{t_{k}^{n}} f\left(X_{s}\right) d s\right) \\
& +2 g\left(X_{k}^{n}\right) \Delta_{i r}^{(2)} B_{k}^{n}\left(\Delta t_{k}^{n} \int_{t_{k}^{n}}^{t_{k+1}^{n}} f\left(X_{s}\right) d s-\Delta t_{k+1}^{n} \int_{t_{k-1}^{n}}^{t_{k}^{n}} f\left(X_{s}\right) d s\right) \\
& +\left(\Delta t_{k}^{n} \int_{t_{k}^{n}}^{t_{k+1}^{n}}\left[g\left(X_{s}\right)-g\left(X_{k}^{n}\right)\right] d B_{s}\right. \\
& \left.\quad-\Delta t_{k+1}^{n} \int_{t_{k-1}^{n}}^{t_{k}^{n}}\left[g\left(X_{s}\right)-g\left(X_{k}^{n}\right)\right] d B_{s}\right)^{2} \\
& +2 g\left(X_{k}^{n}\right) \Delta_{i r}^{(2)} B_{k}^{n}\left(\Delta t_{k}^{n} \int_{t_{k}^{n}}^{t_{k+1}^{n}}\left[g\left(X_{s}\right)-g\left(X_{k}^{n}\right)\right] d B_{s}\right. \\
& \left.\quad-\Delta t_{k+1}^{n} \int_{t_{k-1}^{n}}^{t_{k}^{n}}\left[g\left(X_{s}\right)-g\left(X_{k}^{n}\right)\right] d B_{s}\right) \\
& +g^{2}\left(X_{k}^{n}\right)\left(\Delta_{i r}^{(2)} B_{k}^{n}\right)^{2}=\sum_{i=1}^{6} I_{n, k}^{(i)} .
\end{aligned}
$$

The Love-Young inequality and simple calculations yield

$$
\begin{aligned}
I_{n, k}^{(1)} \leqslant & 2 m_{n}^{3} \int_{t_{k}^{n}}^{t_{k+1}^{n}} f^{2}\left(X_{s}\right) d s+2 m_{n}^{3} \int_{t_{k-1}^{n}}^{t_{k}^{n}} f^{2}\left(X_{s}\right) d s \\
\left|I_{n, k}^{(2)}\right| \leqslant & 4 C_{p, p}\left|g^{\prime}\right|_{\infty} m_{n}^{2} \max _{1 \leqslant k \leqslant N_{n}}\left[V_{p}\left(B ;\left[t_{k-1}^{n}, t_{k}^{n}\right]\right)\right] V_{p}(X ;[0, T]) \\
& \times\left[\int_{t_{k}^{n}}^{t_{k+1}^{n}}\left|f\left(X_{s}\right)\right| d s+\int_{t_{k-1}^{n}}^{t_{k}^{n}}\left|f\left(X_{s}\right)\right| d s\right] \\
\left|I_{n, k}^{(3)}\right| \leqslant & 2 m_{n} \max _{1 \leqslant k \leqslant N_{n}-1}\left|\Delta_{i r}^{(2)} B_{k}\right| \sup _{t \leqslant T}\left|g\left(X_{t}\right)\right| \\
& \times\left[\int_{t_{k}^{n}}^{t_{k+1}^{n}}\left|f\left(X_{s}\right)\right| d s+\int_{t_{k-1}^{n}}^{t_{k}^{n}}\left|f\left(X_{s}\right)\right| d s\right] \\
\left|I_{n, k}^{(4)}\right| \leqslant \leqslant & 2 C_{p, p}^{2}\left|g^{\prime}\right|_{\infty}^{2} m_{n}^{2}\left[V_{p}^{2}\left(X ;\left[t_{k}^{n}, t_{k+1}^{n}\right]\right) V_{p}^{2}\left(B ;\left[t_{k}^{n}, t_{k+1}^{n}\right]\right)\right. \\
& \left.+V_{p}^{2}\left(X ;\left[t_{k-1}^{n}, t_{k}^{n}\right]\right) V_{p}^{2}\left(B ;\left[t_{k-1}^{n}, t_{k}^{n}\right]\right)\right] \\
\left|I_{n, k}^{(5)}\right| \leqslant & 2 C_{p, p}\left|g^{\prime}\right|_{\infty} m_{n} \max _{1 \leqslant k \leqslant N_{n}-1}\left|\Delta_{i r}^{(2)} B_{k}^{n}\right| \sup _{t \leqslant T}\left|g\left(X_{t}\right)\right| \\
\times & \left\{V_{p}\left(X ;\left[t_{k}, t_{k+1}\right]\right) V_{p}\left(B ;\left[t_{k}^{n}, t_{k+1}^{n}\right]\right)\right. \\
& \left.\quad+V_{p}\left(X ;\left[t_{k-1}^{n}, t_{k}^{n}\right]\right) V_{p}\left(B ;\left[t_{k-1}^{n}, t_{k}^{n}\right]\right)\right\}
\end{aligned}
$$


Set

$$
\mu_{k}^{n}=2 \frac{1}{\left(\Delta t_{k}^{n}\right)^{1 / 2+H}\left(\Delta t_{k+1}^{n}\right)^{1 / 2+H}\left(\Delta t_{k}^{n}+\Delta t_{k+1}^{n}\right)} .
$$

Note that $\mu_{k}^{n} \leqslant \frac{1}{p_{n}^{2+2 H}}$ and

$$
\max _{1 \leqslant k \leqslant N_{n}-1}\left|\Delta_{i r}^{(2)} B_{k}^{n}\right| \leqslant 2 m_{n} \max _{1 \leqslant k \leqslant N_{n}}\left|\Delta B_{k}^{n}\right| \leqslant 2 m_{n}^{1+1 / p} L_{T}^{H, 1 / p} .
$$

Thus

$$
\begin{aligned}
\sum_{k=1}^{N_{n}-1} \mu_{k}^{n} \Delta t_{k} \cdot I_{n, k}^{(1)} \leqslant & 4 \frac{m_{n}^{4}}{p_{n}^{2+2 H}} \int_{0}^{T} f^{2}\left(X_{s}\right) d s \\
\sum_{k=1}^{N_{n}-1} \mu_{k}^{n} \Delta t_{k} \cdot I_{n, k}^{(2)} \leqslant & 8 C_{p, p}\left|g^{\prime}\right|_{\infty} \frac{m_{n}^{3}}{p_{n}^{2+2 H}} \max _{1 \leqslant k \leqslant N_{n}} V_{p}\left(B ;\left[t_{k-1}^{n}, t_{k}^{n}\right]\right) \\
& \times V_{p}(X ;[0, T]) \int_{0}^{T}\left|f\left(X_{s}\right)\right| d s
\end{aligned}
$$

$$
\begin{aligned}
\sum_{k=1}^{N_{n}-1} \mu_{k}^{n} \Delta t_{k} \cdot I_{n, k}^{(3)} \leqslant & 8 \frac{m_{n}^{3}}{p_{n}^{2+2 H}} \max _{1 \leqslant k \leqslant N_{n}}\left|\Delta B_{k}^{n}\right| \sup _{t \leqslant T}\left|g\left(X_{t}\right)\right| \int_{0}^{T}\left|f\left(X_{s}\right)\right| d s, \\
\sum_{k=1}^{N_{n}-1} \mu_{k}^{n} \Delta t_{k} \cdot I_{n, k}^{(4)} \leqslant & 4 C_{p, p}^{2}\left|g^{\prime}\right|_{\infty}^{2} \frac{m_{n}^{3}}{p_{n}^{2+2 H}} \max _{1 \leqslant k \leqslant N_{n}} V_{p}\left(B ;\left[t_{k-1}^{n}, t_{k}^{n}\right]\right) V_{p}^{2}(X ;[0, T]) \\
& \times V_{p}(B ;[0, T]), \\
\sum_{k=1}^{N_{n}-1} \mu_{k}^{n} \Delta t_{k}^{n} \cdot I_{n, k}^{(5)} \leqslant & 8 C_{p, p}\left|g^{\prime}\right|_{\infty} \frac{m_{n}^{3}}{p_{n}^{2+2 H}} \max _{1 \leqslant k \leqslant N_{n}}\left|\Delta B_{k}^{n}\right| \sup _{t \leqslant T}\left|g\left(X_{t}\right)\right| \\
& \times V_{p}(X ;[0, T]) V_{p}\left(B^{H} ;[0, T]\right) .
\end{aligned}
$$

The inequalities obtained above are valid for all $p>1 / H$. By Definition 2.1

$$
\frac{m_{n}^{3+1 / p}}{p_{n}^{2+2 H}} \longrightarrow 0, \quad \frac{m_{n}^{4}}{p_{n}^{2+2 H}} \longrightarrow 0, \quad \frac{m_{n}^{3+2 / p}}{p_{n}^{2+2 H}} \longrightarrow 0 \quad \text { as } n \rightarrow \infty,
$$

if $3+1 / p-2-2 H>0$ and $3+2 / p-2-2 H>0$. Such a value of $p$ can 
always be chosen. Thus

$$
\sum_{j=1}^{5} \sum_{k=1}^{N_{n}-1} \mu_{k}^{n} \Delta t_{k}^{n} \cdot I_{n, k}^{(j)} \stackrel{\text { a.s.s. }}{\longrightarrow} 0 \quad \text { as } n \rightarrow \infty
$$

and

$$
V_{\pi_{n}}^{(2)}(X, 2)-\sum_{k=1}^{N_{n}-1} \mu_{k}^{n} \Delta t_{k}^{n} g^{2}\left(X_{k}^{n}\right)\left(\Delta_{i r}^{(2)} B_{k}^{n}\right)^{2} \stackrel{\text { a.s. }}{\longrightarrow} 0 \quad \text { as } n \rightarrow \infty .
$$

Denote $r^{n}(t)=\max \left\{k: t_{k}^{n} \leqslant t\right\}$ and assume that

$$
\begin{aligned}
V_{\pi_{n}}^{(2)}(X, 2)_{t} & =2 \sum_{k=1}^{r^{n}(t)-1} \frac{\Delta t_{k+1}^{n}\left(\Delta_{i r}^{(2)} X_{k}^{n}\right)^{2}}{\left(\Delta t_{k}^{n}\right)^{1 / 2+H}\left(\Delta t_{k+1}^{n}\right)^{1 / 2+H}\left(\Delta t_{k}^{n}+\Delta t_{k+1}^{n}\right)} \\
& =: 2 \sum_{k=1}^{r^{n}(t)-1} \mu_{k}^{n} \Delta t_{k+1}^{n}\left(\Delta_{i r}^{(2)} X_{k}^{n}\right)^{2}
\end{aligned}
$$

From the results obtained in Bégyn (2006) it is easy to see that for every $t \in$ $[0, T]$

$$
V_{\pi_{n}}^{(2)}\left(B^{H}, 2\right)_{t}-\mathbf{E} V_{\pi_{n}}^{(2)}\left(B^{H}, 2\right)_{t} \stackrel{\text { a.s. }}{\longrightarrow} 0 \quad \text { as } n \rightarrow \infty .
$$

Let's show that

$$
\mathbf{E} V_{\pi_{n}}^{(2)}\left(B^{H}, 2\right)_{t}=2 \sum_{k=1}^{r^{n}(t)-1} h\left(\ell_{k}\right) \Delta t_{k+1}^{n}
$$

Obviously,

$$
\mathbf{E} V_{\pi_{n}}^{(2)}(X, 2)_{t}=2 \sum_{k=1}^{r^{n}(t)-1} \mu_{k}^{n} \Delta t_{k+1}^{n} \mathbf{E}\left(\Delta_{i r}^{(2)} X_{k}^{n}\right)^{2}
$$

and simple calculations yield

$$
\begin{aligned}
\mathbf{E}\left(\Delta_{i r}^{(2)} X_{k}^{n}\right)^{2}= & \mathbf{E}\left(\Delta t_{k} \Delta B_{k+1}^{H}-\Delta t_{k+1} \Delta B_{k}^{H}\right)^{2}=\left(\Delta t_{k}\right)^{2} \cdot\left(\Delta t_{k+1}\right)^{2 H} \\
& -2 \Delta t_{k} \Delta t_{k+1} \mathbf{E} \Delta B_{k}^{H} \Delta B_{k+1}^{H}+\left(\Delta t_{k+1}\right)^{2} \cdot\left(\Delta t_{k}\right)^{2 H} \\
= & \left(\Delta t_{k}\right)^{2} \cdot\left(\Delta t_{k+1}\right)^{2 H}+\left(\Delta t_{k+1}\right)^{2} \cdot\left(\Delta t_{k}\right)^{2 H}
\end{aligned}
$$




$$
\begin{aligned}
& -\Delta t_{k} \Delta t_{k+1}\left[\left(\Delta t_{k+1}+\Delta t_{k}\right)^{2 H}-\left(\Delta t_{k+1}\right)^{2 H}-\left(\Delta t_{k}\right)^{2 H}\right] \\
= & \Delta t_{k} \Delta t_{k+1}\left(\Delta t_{k}+\Delta t_{k+1}\right)\left[\left(\Delta t_{k+1}\right)^{2 H-1}+\left(\Delta t_{k}\right)^{2 H-1}\right. \\
& \left.-\left(\Delta t_{k+1}+\Delta t_{k}\right)^{2 H-1}\right] .
\end{aligned}
$$

Therefore

$$
\begin{aligned}
& \mathbf{E} V_{\pi_{n}}^{(2)}(X, 2)_{t} \\
& \quad=2 \sum_{k=1}^{r^{n}(t)-1} \Delta t_{k+1} \frac{\left(\Delta t_{k+1}\right)^{2 H-1}-\left(\Delta t_{k+1}+\Delta t_{k}\right)^{2 H-1}+\left(\Delta t_{k}\right)^{2 H-1}}{\left(\Delta t_{k}^{n}\right)^{H-1 / 2} \cdot\left(\Delta t_{k+1}^{n}\right)^{H-1 / 2}} \\
& \quad=2 \sum_{k=1}^{r^{n}(t)-1} \Delta t_{k+1} \frac{1-\left(\ell_{k}+1\right)^{2 H-1}+\ell_{k}^{2 H-1}}{\ell_{k}^{H-1 / 2}}=2 \sum_{k=1}^{r^{n}(t)-1} h\left(\ell_{k}\right) \Delta t_{k+1}^{n} .
\end{aligned}
$$

Further it'll be proved that

$$
\begin{gathered}
\sup _{t \leqslant T}\left|S_{t}^{n}\right| \stackrel{\text { a.s. }}{\longrightarrow} 0 \quad \text { as } n \rightarrow \infty, \quad \text { where } \\
S_{t}^{n}=2 \sum_{k=1}^{r^{n}(t)-1}\left[\mu_{k}^{n} \Delta t_{k+1}^{n}\left(\Delta_{i r}^{(2)} B_{k}^{H}\right)^{2}-h\left(\ell_{k}\right) \Delta t_{k+1}^{n}\right], \quad t \in[0, T] .
\end{gathered}
$$

Let $\left(s_{j}^{m}\right), 0 \leqslant j \leqslant m, m \geqslant 1$, be a sequence of partitions of the interval $[0, T], 0=s_{0}^{m}<s_{1}^{m}<\ldots<s_{m}^{m}=T$, such that $\max _{1 \leqslant j \leqslant m}\left(s_{j}^{m}-s_{j-1}^{m}\right) \rightarrow 0$ as $m \rightarrow \infty$. To prove (2.3) it is suffices to show that for every such sequence $\left(s_{j}^{m}\right)$ (see Lemma 5 in Liptser, Shiryaev (1989) p. 556-557)

$\lim _{m \rightarrow \infty} \limsup _{n \rightarrow \infty} \max _{1 \leqslant j \leqslant m-1}\left|\sum_{k=1}^{r^{n}\left(s_{j+1}^{m}\right)-1} h\left(\ell_{k}\right) \Delta t_{k+1}^{n}-\sum_{k=1}^{r^{n}\left(s_{j}^{m}\right)-1} h\left(\ell_{k}\right) \Delta t_{k+1}^{n}\right|=0$.

Note that the function $h$ is continuous and bounded, i. e. $0 \leqslant h(\lambda) \leqslant 1$ for $\lambda \geqslant 0$. Thus

$$
\begin{aligned}
& \max _{1 \leqslant j \leqslant m-1}\left|\sum_{k=1}^{r^{n}\left(s_{j+1}^{m}\right)-1} h\left(\ell_{k}\right) \Delta t_{k+1}^{n}-\sum_{k=1}^{r^{n}\left(s_{j}^{m}\right)-1} h\left(\ell_{k}\right) \Delta t_{k+1}^{n}\right| \\
& \leqslant \max _{1 \leqslant j \leqslant m-1}\left|\rho^{n}\left(s_{j+1}^{m}\right)-\rho^{n}\left(s_{j}^{m}\right)\right|,
\end{aligned}
$$


where $\rho^{n}(t)=\max \left\{t_{k}^{n}: t_{k}^{n} \leqslant t\right\}$, and for sufficiently large $n$ the value of $m$ can be chosen such that the right side of inequality is a diminutive value. Therefore $S_{t}^{n}$ is uniformly bounded for almost all $\omega$ and by the Helly-Bray theorem

$$
\begin{gathered}
\sum_{k=1}^{N_{n}-1} g^{2}\left(X_{k}^{n}\right)\left[\mu_{k}^{n} \Delta t_{k}^{n}\left(\Delta_{i r}^{(2)} B_{k}^{n}\right)^{2}-2 h\left(\ell_{k}\right) \Delta t_{k+1}^{n}\right] \\
=\int_{0}^{T} g^{2}\left(X_{t}\right) d S_{t}^{n} \stackrel{\text { a.s. }}{\longrightarrow} 0 \quad \text { as } \quad n \rightarrow \infty
\end{gathered}
$$

Then

$$
\sum_{k=1}^{N_{n}-1} g^{2}\left(X_{k}^{n}\right) h\left(\ell_{k}\right) \Delta t_{k+1}^{n}=\int_{0}^{T} g^{2}\left(X_{t}^{\pi_{n}}\right) h\left(\ell_{n}(t)\right) d t
$$

and

$$
\begin{aligned}
\int_{0}^{T} & \left|g^{2}\left(X_{t}\right) h(\ell(t))-g^{2}\left(X_{t}^{\pi_{n}}\right) h\left(\ell_{n}(t)\right)\right| d t \\
\leqslant & T \sup _{t \leqslant T}\left|g^{2}\left(X_{t}\right)\right| \sup _{t \leqslant T}\left|h\left(\ell_{n}(t)\right)-h(\ell(t))\right| \\
& +T \sup _{t \leqslant T}\left|g^{2}\left(X_{t}\right)-g^{2}\left(X_{t}^{\pi_{n}}\right)\right| \longrightarrow 0 \quad \text { as } \quad n \rightarrow \infty
\end{aligned}
$$

which completes the proof.

\subsection{The Milstein approximation}

For a process $\left\{X_{t}\right\}$, its Milstein approximation at the points $t_{k}^{n}, k=$ $1, \ldots, n$ is defined as

$$
Y_{k}^{n}=Y_{k-1}^{n}+f\left(Y_{k-1}^{n}\right) \Delta t_{k}+g\left(Y_{k-1}^{n}\right) \Delta B_{k}^{H}+\frac{1}{2} g\left(Y_{k-1}^{n}\right) g^{\prime}\left(Y_{k-1}^{n}\right)\left(\Delta B_{k}^{H}\right)^{2},
$$

where $g^{\prime}$ denotes the derivative of $g$ and $Y_{0}^{n}=X_{0}$.

The following result allows us to assert that if we replace the solution by its Milstein approximation the estimators of $H$ remain consistent. 
2.9 Theorem. Let $f$ be a Lipschitz-continuos function and let $g \in \mathcal{C}^{1+\alpha}, \frac{1}{H}-$ $1<\alpha \leqslant 1$. Define

$$
\begin{aligned}
\widehat{H}_{n}^{(1), M} & :=\frac{1}{2}-\frac{1}{2 \ln \left(N_{2 n} / N_{n}\right)} \ln \frac{V_{N_{2 n}}^{(1)}\left(Y^{n}, 2\right)}{V_{N_{n}}^{(1)}\left(Y^{n}, 2\right)} \\
\widehat{H}_{n}^{(2), M} & :=\frac{1}{2}-\frac{1}{2 \ln \left(N_{2 n} / N_{n}\right)} \ln \frac{V_{N_{2 n}}^{(2)}\left(Y^{n}, 2\right)}{V_{N_{n}}^{(2)}\left(Y^{n}, 2\right)} .
\end{aligned}
$$

If the subdivisions of the interval $[0, T]$ satisfy the conditions of Theorem 2.6, then $\widehat{H}_{n}^{(1), M}-H \stackrel{\text { a.s. }}{\longrightarrow} 0$ as $n \rightarrow \infty$. If subdivisions of the interval $[0, T]$ satisfy the conditions of Theorem 2.8 , then $\widehat{H}_{n}^{(2), M}-H \stackrel{\text { a.s }}{\longrightarrow} 0$ as $n \rightarrow \infty$.

Proof of Theorem 2.9. We consider only the second order increments of the Milstein approximation of the solution $X$ of the equation (1.1). A consideration of the first order increments of the Milstein approximation is quite similar. Note that at subdivision points $\left\{t_{k}^{n}\right\}$ the Milstein approximation can be written in the form

$$
\begin{aligned}
Y^{n}\left(t_{k}^{n}\right)= & Y^{n}\left(t_{k-1}^{n}\right)+f\left(Y^{n}\left(t_{k-1}^{n}\right)\right) \cdot \Delta t_{k}^{n}+g\left(Y^{n}\left(t_{k-1}^{n}\right)\right) \cdot \Delta B_{k}^{H, n} \\
& +\frac{1}{2} g\left(Y^{n}\left(t_{k-1}^{n}\right)\right) g^{\prime}\left(Y^{n}\left(t_{k-1}^{n}\right)\right) \cdot\left(\Delta B_{k}^{H, n}\right)^{2},
\end{aligned}
$$

$Y^{n}(0)=X(0)=\xi$. Denote $Y_{k}^{n}=Y^{n}\left(t_{k}^{n}\right)$ and $g g^{\prime}\left(Y_{k}^{n}\right)=g\left(Y_{k}^{n}\right) g^{\prime}\left(Y_{k}^{n}\right)$.

Then

$$
\begin{aligned}
& \Delta_{i r}{ }^{(2)} Y_{k}^{n}=\Delta t_{k}^{n} Y_{k+1}^{n}+\Delta t_{k+1}^{n} Y_{k-1}^{n}-\left(\Delta t_{k}^{n}+\Delta t_{k+1}^{n}\right) Y_{k}^{n} \\
& =\Delta t_{k}^{n}\left(Y_{k+1}^{n}-Y_{k}^{n}\right)-\Delta t_{k+1}^{n}\left(Y_{k}^{n}-Y_{k-1}^{n}\right) \\
& =\Delta t_{k}^{n}\left[f\left(Y_{k}^{n}\right) \Delta t_{k+1}^{n}+g\left(Y_{k}^{n}\right) \Delta B_{k+1}^{H, n}+\frac{1}{2} g g^{\prime}\left(Y_{k}^{n}\right)\left(\Delta B_{k+1}^{H, n}\right)^{2}\right] \\
& \quad-\Delta t_{k+1}^{n}\left[f\left(Y_{k-1}^{n}\right) \Delta t_{k}^{n}+g\left(Y_{k-1}^{n}\right) \Delta B_{k}^{H, n}+\frac{1}{2} g g^{\prime}\left(Y_{k-1}\right)\left(\Delta B_{k}^{H, n}\right)^{2}\right] .
\end{aligned}
$$

Therefore $\Delta_{i r}^{(2)} Y_{k}^{n}$ can be rewritten as

$$
\begin{aligned}
\Delta_{i r}{ }^{(2)} Y_{k}^{n} & =\Delta t_{k}^{n} \Delta t_{k+1}^{n}\left[f\left(Y_{k}^{n}\right)-f\left(Y_{k-1}^{n}\right)\right] \\
& +\left[g\left(Y_{k}^{n}\right) \Delta t_{k}^{n} \Delta B_{k+1}^{H, n}-g\left(Y_{k-1}^{n}\right) \Delta t_{k+1}^{n} \Delta B_{k}^{H, n}\right] \\
& +\frac{1}{2}\left[g g^{\prime}\left(Y_{k}^{n}\right) \Delta t_{k}^{n}\left(\Delta B_{k+1}^{H, n}\right)^{2}-g g^{\prime}\left(Y_{k-1}^{n}\right) \Delta t_{k+1}^{n}\left(\Delta B_{k}^{H, n}\right)^{2}\right]
\end{aligned}
$$


and, therefore

$$
\begin{aligned}
& \Delta_{i r}{ }^{(2)} Y_{k}^{n}=[ {\left[f\left(Y_{k}^{n}\right)-f\left(Y_{k-1}^{n}\right)\right] \Delta t_{k}^{n} \Delta t_{k+1}^{n} } \\
&\left.+\left[g\left(Y_{k}^{n}\right)-g\left(Y_{k-1}^{n}\right)\right] \Delta t_{k+1}^{n} \Delta B_{k}^{H, n}\right] \\
&+\frac{1}{2}\left[g g^{\prime}\left(Y_{k}^{n}\right) \Delta t_{k}^{n}\left(\Delta B_{k+1}^{H, n}\right)^{2}-g g^{\prime}\left(Y_{k-1}^{n}\right) \Delta t_{k+1}^{n}\left(\Delta B_{k}^{H, n}\right)^{2}\right] \\
&+ \\
& \quad g\left(Y_{k}^{n}\right) \Delta_{i r}^{(2)} B_{k}^{H, n} \\
&=I_{n, k}^{(1)}+\frac{1}{2} I_{n, k}^{(2)}+g\left(Y_{k}^{n}\right) \Delta_{i r}^{(2)} B_{k}^{H, n} .
\end{aligned}
$$

\section{Further}

$$
\begin{aligned}
& \left(I_{n, k}^{(1)}\right)^{2} \leqslant 2 \max _{1 \leqslant k \leqslant N_{n}}\left|Y_{k}^{n}-Y_{k-1}^{n}\right|^{2}\left[m_{n}^{4} L^{2}+m_{n}^{2}\left|g^{\prime}\right|_{\infty}^{2} \max _{1 \leqslant k \leqslant N_{n}}\left|\Delta B_{k}^{H, n}\right|^{2}\right], \\
& \left|I_{n, k}^{(1)}\left[g\left(Y_{k}^{n}\right) \Delta_{i r}^{(2)} B_{k}^{H, n}\right]\right| \leqslant \\
& \leqslant 2 m_{n}^{2} \max _{1 \leqslant k \leqslant N_{n}}\left|g\left(Y_{k}^{n}\right)\right| \max _{1 \leqslant k \leqslant N_{n}}\left|Y_{k}^{n}-Y_{k-1}^{n}\right| \max _{1 \leqslant k \leqslant N_{n}}\left|\Delta B_{k}^{H, n}\right| \\
& \quad \times\left[L m_{n}+\left|g^{\prime}\right|_{\infty} \max _{1 \leqslant k \leqslant N_{n}}\left|\Delta B_{k}^{H, n}\right|\right], \\
& \left(I_{n, k}^{(2)}\right)^{2} \leqslant 4 m_{n}^{2} \max _{1 \leqslant k \leqslant N_{n}}\left|g g^{\prime}\left(Y_{k}^{n}\right)\right|^{2} \max _{1 \leqslant k \leqslant N_{n}}\left|\Delta B_{k}^{H, n}\right|^{4} .
\end{aligned}
$$

By using the inequality (1.8) we get

$$
\begin{aligned}
& \sum_{k=1}^{N_{n}-1} \mu_{k}^{n} \Delta t_{k}^{n}\left(I_{n, k}^{(1)}\right)^{2} \leqslant \\
& \leqslant 2 T \max _{1 \leqslant k \leqslant N_{n}}\left|Y_{k}^{n}-Y_{k-1}^{n}\right|^{2}\left[\frac{m_{n}^{4} L^{2}}{p_{n}^{2+2 H}}+\frac{m_{n}^{2+2 / p}\left|g^{\prime}\right|_{\infty}^{2}}{p_{n}^{2+2 H}}\left(L_{T}^{H, 1 / p}\right)^{2}\right] \\
& 2 \sum_{k=1}^{N_{n}-1} \mu_{k}^{n} \Delta t_{k}^{n}\left|I_{n, k}^{(1)}\left[g\left(Y_{k}^{n}\right) \Delta_{i r}^{(2)} B_{k}^{H, n}\right]\right| \leqslant \\
& \leqslant 4 T \max _{1 \leqslant k \leqslant N_{n}}\left|g\left(Y_{k}^{n}\right)\right| \max _{1 \leqslant k \leqslant N_{n}}\left|Y_{k}^{n}-Y_{k-1}^{n}\right| L_{T}^{H, 1 / p} \\
& \times\left[\frac{m_{n}^{3+1 / p} L}{p_{n}^{2+2 H}}+\frac{m_{n}^{2+2 / p}\left|g^{\prime}\right|_{\infty}}{p_{n}^{2+2 H}} L_{T}^{H, 1 / p}\right]
\end{aligned}
$$




$$
\frac{1}{4} \sum_{k=1}^{N_{n}-1} \mu_{k}^{n} \Delta t_{k}^{n}\left(I_{n, k}^{(2)}\right)^{2} \leqslant T \frac{m_{n}^{2+4 / p}}{p_{n}^{2+2 H}} \max _{1 \leqslant k \leqslant N_{n}}\left|g g^{\prime}\left(Y_{k}^{n}\right)\right|^{2}\left(L_{T}^{H, 1 / p}\right)^{4}
$$

and

$$
\begin{aligned}
& \sum_{k=1}^{N_{n}-1} \mu_{k}^{n} \Delta t_{k}^{n}\left|\left[I_{n, k}^{(1)}+g\left(Y_{k}^{n}\right) \Delta_{i r}^{(2)} B_{k}^{H, n}\right] I_{n, k}^{(2)}\right| \\
& \quad \leqslant \sqrt{2 \sum_{k=1}^{N_{n}-1} \mu_{k}^{n} \Delta t_{k}^{n}\left[\left|I_{n, k}^{(1)}\right|^{2}+g^{2}\left(Y_{k}^{n}\right)\left(\Delta_{i r}^{(2)} B_{k}^{H, n}\right)^{2}\right] \sum_{k=1}^{N_{n}-1} \mu_{k}^{n} \Delta t_{k}^{n}\left|I_{n, k}^{(2)}\right|^{2}}
\end{aligned}
$$

It is easily verified that

$$
\begin{aligned}
\max _{1 \leqslant k \leqslant N_{n}}\left|Y_{k}^{n}-Y_{k-1}^{n}\right| \leqslant & \max _{1 \leqslant k \leqslant N_{n}}\left|f\left(Y_{k-1}^{n}\right)\right| \cdot \Delta t_{k}^{n}+\max _{1 \leqslant k \leqslant N_{n}}\left|g\left(Y_{k-1}^{n}\right) \cdot \Delta B_{k}^{H, n}\right| \\
& +\frac{1}{2} \max _{1 \leqslant k \leqslant N_{n}}\left|g g^{\prime}\left(Y_{k-1}^{n}\right)\left(\Delta B_{k}^{H, n}\right)^{2}\right| \\
\leqslant & m_{n}^{1 / p}\left[\max _{1 \leqslant k \leqslant N_{n}}\left|f\left(Y_{k-1}^{n}\right)\right|+\max _{1 \leqslant k \leqslant N_{n}}\left|g\left(Y_{k-1}^{n}\right)\right| \cdot L_{T}^{H, 1 / p}\right] \\
& +m_{n}^{2 / p} \max _{1 \leqslant k \leqslant N_{n}}\left|g g^{\prime}\left(Y_{k-1}^{n}\right)\right| \cdot\left(L_{T}^{H, 1 / p}\right)^{2} .
\end{aligned}
$$

Since $\sup _{n} V_{p}\left(Y^{n} ;[0, T]\right)<\infty$ (see Kubilius (1999)), the functions $g\left(Y_{k}^{n}\right)$, $g^{\prime}\left(Y_{k}^{n}\right)$ and $g g^{\prime}\left(Y_{k}^{n}\right)$ are uniformly bounded. Thus

$$
V_{\pi_{n}}^{(2)}\left(Y^{n}, 2\right)-\sum_{k=1}^{N_{n}-1} \mu_{k}^{n} \Delta t_{k+1}^{n} g^{2}\left(Y_{k}^{n}\right)\left(\Delta_{i r}^{(2)} B_{k}^{H, n}\right)^{2} \stackrel{\text { a.s. }}{\longrightarrow} 0 \quad \text { as } \quad n \rightarrow \infty .
$$

To complete the proof, it suffices to observe that

$$
\begin{aligned}
& \left|\sum_{k=1}^{N_{n}-1} \mu_{k}^{n} \Delta t_{k+1}^{n}\left[g^{2}\left(X_{k}^{n}\right)-g^{2}\left(Y_{k}^{n}\right)\right]\left(\Delta_{i r}^{(2)} B_{k}^{H, n}\right)^{2}\right| \\
& \quad \leqslant \max _{1 \leqslant k \leqslant N_{n}}\left|g^{2}\left(X_{k}^{n}\right)-g^{2}\left(Y_{k}^{n}\right)\right| \sum_{k=1}^{N_{n}-1} \mu_{k}^{n} \Delta t_{k+1}^{n}\left(\Delta_{i r}^{(2)} B_{k}^{H, n}\right)^{2}
\end{aligned}
$$

and the last term tends to 0 as $n \rightarrow \infty$. To prove this we use some well known 
results. In Bégyn (2005) it was proved that

$$
\sum_{k=1}^{N_{n}-1} \mu_{k}^{n} \Delta t_{k+1}^{n}\left(\Delta_{i r}^{(2)} B_{k}^{H, n}\right)^{2} \stackrel{\text { a.s. }}{\longrightarrow} 2 \int_{0}^{T} h(\ell(s)) d s \quad \text { as } \quad n \rightarrow \infty .
$$

The $p$-variation distance between the solution and its Milstein approximation was estimated in Kubilius (1999) (see also Kubilius (2000)). It follows from this that

$$
\sup _{t \leqslant T}\left|Y_{t}^{n}-X_{t}^{n}\right| \stackrel{\text { a.s. }}{\longrightarrow} 0 \quad \text { as } \quad n \rightarrow \infty .
$$

Consequently, the limit behavior of the second order increments of the Milstein approximation is the same as the limit behavior of the solution of SDE. Thus, the convergence $\widehat{H}_{n}^{(2), M}-H \stackrel{\text { a.s. }}{\rightarrow} 0$ holds as $n \rightarrow \infty$.

\subsection{The increment ratios statistic}

The increment ratios (IR) statistic is defined as

$$
R^{p, n}(f)=\frac{1}{n-p} \sum_{k=0}^{n-p-1} \frac{\left|\Delta_{k}^{p, n} f+\Delta_{k+1}^{p, n} f\right|}{\left|\Delta_{k}^{p, n} f\right|+\left|\Delta_{k+1}^{p, n} f\right|}
$$

where $\Delta_{k}^{p, n} f$ denotes the $p$-order increments of a real-valued function $f$ at $t_{k}^{n}$, $p=1,2, \ldots, k=0,1, \ldots, n-p$, that is,

$$
\Delta_{k}^{1, n} f=f\left(t_{k+1}^{n}\right)-f\left(t_{k}^{n}\right), \quad \Delta_{k}^{p, n} f=\Delta_{k}^{1, n} \Delta_{k}^{p-1, n} f .
$$

J. M. Bardet and D. Surgailis (2010) showed that if $X$ is a fractional Brownian motion $\left(B^{H}\right)$ with parameter $H \in(0,1)$, then

$$
\begin{gathered}
R^{p, n}(f) \stackrel{\text { a.s. }}{\rightarrow} \Lambda_{p}(H) \quad \text { as } n \rightarrow \infty, p=1,2, \\
\text { where } \quad \Lambda_{p}(H)=\mathbf{E} \frac{\left|\Delta_{0}^{p} B^{H}+\Delta_{1}^{p} B^{H}\right|}{\left|\Delta_{0}^{p} B^{H}\right|+\left|\Delta_{1}^{p} B^{H}\right|} .
\end{gathered}
$$

The $R^{2, n}(f)$ statistic is better suited for practical purposes than $R^{1, n}(f)$ since the error arising from approximating $\Lambda_{2}(H)$ with a line is considerably lower than that of $\Lambda_{1}(H)$. Fig. 2.1 presents the graph of $\Lambda_{2}(H)$ as well as the graph of $R^{2,100}\left(B^{H}\right)$ averaged over 50 sample paths.

In the recent years it has been proposed by several authors to replace the 


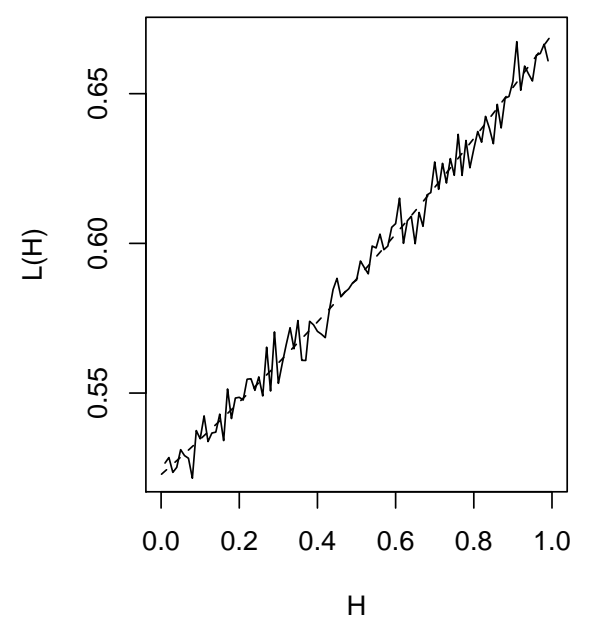

Fig. 2.1. Graphs of $\Lambda_{2}(H)$ and $R^{2,100}\left(B^{H}\right)$

classic Black-Scholes model, based on the standard geometric Brownian motion, with its fractional counterpart. This would enable the model to handle the possible existence of long-range dependance in the observed data. In this section it is shown that the convergence (2.4) holds for $H \in(1 / 2,7 / 8)$ when $p=1$ and $H \in(1 / 2,1)$ when $p=2$ if $X$ is the solution of the fractional Black-Scholes equation.

2.10 Theorem. Let $B^{H}=\left\{B_{t}^{H} ; t \in[0,1]\right\}$ denote the fractional Brownian motion with parameter $H$ and let $X$ be the solution of the fractional BlackScholes equation (or, in other words, the fractional geometric Brownian motion)

$$
d X_{t}=\mu X_{t} d t+\sigma X_{t} d B_{t}^{H}, \quad \mu, \sigma, X_{0} \in \mathbb{R}
$$

observed at times $t_{k}^{n}=\frac{k}{2^{n}}, k=0,1, \ldots, 2^{n}$. Then

$$
R^{p, n}(X) \stackrel{\text { a.s }}{\longrightarrow} \Lambda_{p}(H) \quad \text { as } n \rightarrow \infty, p=1,2
$$

for $H \in(1 / 2,7 / 8)$ when $p=1$ and $H \in(1 / 2,1)$ when $p=2$.

The proof of this theorem is based on the following lemma which is a generalization of the corresponding lemma in the paper of J. M. Bardet and D. Surgailis.

2.1 Lemma. Let $\psi\left(x_{1}, x_{2}\right)=\frac{\left|x_{1}+x_{2}\right|}{\left|x_{1}\right|+\left|x_{2}\right|}, x_{1}, x_{2} \in \mathbb{R}$, and let $\left(Z_{1}, Z_{2}\right)$ be a 
Gaussian vector with zero mean and dispersion $\mathbf{E} Z_{i}^{2}=1, i=1,2$. Then for any $r . v . \xi_{i}, i=1,2$,

$$
\mathbf{E}\left|\psi\left(Z_{1}+\xi_{1}, Z_{2}+\xi_{2}\right)-\psi\left(Z_{1}, Z_{2}\right)\right| \leqslant 16 \max _{i=1,2} \sqrt[3]{\mathbf{E} \xi_{i}^{2}}, \quad k \geqslant 1 .
$$

Proof of Lemma 2.1. Let $\delta^{2}=\max _{i=1,2} \mathbf{E} \xi_{i}^{2}$. Denote $U:=\psi\left(Z_{1}+\xi_{1}, Z_{2}+\right.$ $\left.\xi_{2}\right)-\psi\left(Z_{1}, Z_{2}\right)=U_{\delta}+U_{\delta}^{c}$,

$$
\begin{aligned}
U_{\delta} & :=U \mathbf{1}_{A_{\delta}}=\left(\psi\left(Z_{1}+\xi_{1}, Z_{2}+\xi_{2}\right)-\psi\left(Z_{1}, Z_{2}\right)\right) \mathbf{1}_{A_{\delta}}, \\
U_{\delta}^{c} & :=U \mathbf{1}_{A_{\delta}^{c}}=\left(\psi\left(Z_{1}+\xi_{1}, Z_{2}+\xi_{2}\right)-\psi\left(Z_{1}, Z_{2}\right)\right) \mathbf{1}_{A_{\delta}^{c}},
\end{aligned}
$$

where $\mathbf{1}_{A_{\delta}}$ is the indicator of the event

$$
A_{\delta}:=\left\{\left|Z_{1}\right|>\delta^{2 / 3},\left|Z_{2}\right|>\delta^{2 / 3},\left|\xi_{1}\right|<\delta^{2 / 3} / 2,\left|\xi_{2}\right|<\delta^{2 / 3} / 2\right\}
$$

and $\mathbf{1}_{A_{\delta}^{c}}=1-\mathbf{1}_{A_{\delta}}$ is the indicator of the complementary event $A_{\delta}^{c}$. Clearly,

$$
\begin{aligned}
\mathbf{E}\left|U_{\delta}^{c}\right| \leqslant & 2\left[\mathbf{P}\left(\left|Z_{1}\right|<\delta^{2 / 3}\right)+\mathbf{P}\left(\left|Z_{2}\right|<\delta^{2 / 3}\right)\right. \\
& \left.+\mathbf{P}\left(\left|\xi_{1}\right| \geqslant \delta^{2 / 3} / 2\right)+\mathbf{P}\left(\left|\xi_{2}\right| \geqslant \delta^{2 / 3} / 2\right)\right] \\
\leqslant & \frac{8}{\sqrt{2 \pi}} \delta^{2 / 3}+8 \max _{i=1,2} \frac{\mathbf{E}\left|\xi_{i}\right|^{2}}{\delta^{4 / 3}} \leqslant 12 \delta^{2 / 3} .
\end{aligned}
$$

It remains to estimate $\mathbf{E}\left|U_{\delta}\right|$. By the mean value theorem,

$$
U_{\delta}=\left(\xi_{1} \frac{\partial \psi}{\partial x_{1}}\left(Z_{1}+\theta \xi_{1}, Z_{2}+\theta \xi_{2}\right)+\xi_{2} \frac{\partial \psi}{\partial x_{2}}\left(Z_{1}+\theta \xi_{1}, Z_{2}+\theta \xi_{2}\right)\right) \mathbf{1}_{A_{\delta}},
$$

where $0<\theta(\omega)<1$ and

$$
\begin{aligned}
\left|\frac{\partial \psi}{\partial x_{i}}\left(x_{1}, x_{2}\right)\right| & =\frac{\left|\left(\left|x_{1}\right|+\left|x_{2}\right|\right) \operatorname{sgn}\left(x_{1}+x_{2}\right)-\right| x_{1}+x_{2}\left|\operatorname{sgn}\left(x_{i}\right)\right|}{\left(\left|x_{1}\right|+\left|x_{2}\right|\right)^{2}} \\
& \leqslant \frac{2}{\left|x_{1}\right|+\left|x_{2}\right|} .
\end{aligned}
$$

Thus

$$
\left|\frac{\partial \psi}{\partial x_{i}}\left(Z_{1}+\theta \xi_{1}, Z_{2}+\theta \xi_{2}\right)\right| \mathbf{1}_{A_{\delta}} \leqslant \frac{2}{\left|Z_{1}+\theta \xi_{1}\right|+\left|Z_{2}+\theta \xi_{2}\right|} \mathbf{1}_{A_{\delta}}
$$




$$
\leqslant \frac{2}{\left|Z_{1}+\theta \xi_{1}\right|+\left|Z_{2}+\theta \xi_{2}\right|} \mathbf{1}_{B_{\delta}} \leqslant \frac{2}{\delta^{2 / 3}} \mathbf{1}_{B_{\delta}}
$$

where

$$
\begin{gathered}
B_{\delta}=\left\{\left|Z_{1}+\theta \xi_{1}\right|>\delta^{2 / 3} / 2,\left|Z_{1}+\theta \xi_{1}\right|>\delta^{2 / 3} / 2\right. \\
\left.\left|\xi_{1}\right| \leqslant \delta^{2 / 3} / 2,\left|\xi_{2}\right| \leqslant \delta^{2 / 3} / 2\right\} .
\end{gathered}
$$

Therefore

$$
\begin{aligned}
\mathbf{E}\left|U_{\delta}\right| \leqslant & \mathbf{E}^{1 / 2} \xi_{1}^{2} \cdot \mathbf{E}^{1 / 2}\left[\left|\frac{\partial \psi}{\partial x_{1}}\left(Z_{1}+\theta \xi_{1}, Z_{2}+\theta \xi_{2}\right)\right|^{2} \mathbf{1}_{A_{\delta}}\right] \\
& +\mathbf{E}^{1 / 2} \xi_{2}^{2} \cdot \mathbf{E}^{1 / 2}\left[\left|\frac{\partial \psi}{\partial x_{2}}\left(Z_{1}+\theta \xi_{1}, Z_{2}+\theta \xi_{2}\right)\right|^{2} \mathbf{1}_{A_{\delta}}\right] \leqslant 4 \delta^{2 / 3}
\end{aligned}
$$

and

$$
\mathbf{E}|U| \leqslant 16 \delta^{2 / 3} .
$$

Proof of Theorem 2.10. Let $\Delta t^{n}$ denote the mesh of the subdivision, that is, $\Delta t^{n}:=2^{-n}$. Let $\psi_{k}^{n}=\mu \Delta t^{n}+\sigma \Delta B_{k}^{H, n}$. The fractional geometric Brownian motion (2.5) is $X_{t}=c \exp \left(\mu t+\sigma B_{t}^{H}\right)$. Therefore $R^{1, n}(X)$ can be rewritten as

$$
R^{1, n}(X)=\frac{1}{2^{n}-1} \sum_{k=0}^{2^{n}-2} \frac{\left|\exp \left(\psi_{k+1}^{n}\right)-\exp \left(-\psi_{k}^{n}\right)\right|}{\left|1-\exp \left(-\psi_{k}^{n}\right)\right|+\left|\exp \left(\psi_{k+1}^{n}\right)-1\right|} .
$$

For briefness, let the index $n$ be omitted. Then the Taylor expansion yields

$$
\begin{aligned}
& \exp \left(\psi_{k+1}\right)-1=\sigma \Delta B_{k+1}^{H}+\left(\mu \Delta t+R\left(\mu \Delta t+\sigma \Delta B_{k+1}^{H}\right)\right), \\
& 1-\exp \left(-\psi_{k}\right)=\sigma \Delta B_{k}^{H}+\left(\mu \Delta t-R\left(-\mu \Delta t-\sigma \Delta B_{k}^{H}\right)\right), \\
& \quad \exp \left(\psi_{k+1}\right)-\exp \left(-\psi_{k}\right)=\sigma\left(\Delta B_{k+1}^{H}+\Delta B_{k}^{H}\right)+ \\
& \quad+\left(2 \mu \Delta t+R\left(\mu \Delta t+\sigma \Delta B_{k+1}^{H}\right)-R\left(-\mu \Delta t-\sigma \Delta B_{k}^{H}\right)\right),
\end{aligned}
$$

where

$$
R(x)=\frac{x^{2}}{2} e^{\theta x}, \quad 0<\theta<1 .
$$

Let $Z_{1}(k)=\sigma \Delta B_{k}^{H}, \xi_{1}(k)=\mu \Delta t-R\left(-\mu \Delta t-\sigma \Delta B_{k}^{H}\right), Z_{2}(k)=\sigma \Delta B_{k+1}^{H}$, 
$\xi_{2}(k)=\mu \Delta t+R\left(\mu \Delta t+\sigma \Delta B_{k+1}^{H}\right)$. Obviously

$$
R^{1, n}(X)=\frac{1}{2^{n}-1} \sum_{k=0}^{2^{n}-2} \frac{\left|Z_{1}(k)+\xi_{1}(k)+Z_{2}(k)+\xi_{2}(k)\right|}{\left|Z_{1}(k)+\xi_{1}(k)\right|+\left|Z_{2}(k)+\xi_{2}(k)\right|} .
$$

Define $\psi_{k}:=\mu \Delta t+\sigma \Delta B_{k}^{H}$. Then

$$
\begin{aligned}
\mathbf{E}\left(\xi_{1}(k)\right)^{2} & \leqslant 2(\mu \Delta t)^{2}+\mathbf{E}\left(\psi_{k}^{4} e^{-2 \theta \psi_{k}}\right) \\
& \leqslant 2(\mu \Delta t)^{2}+\sqrt{105\left(\mathbf{E} \psi_{k}^{2}\right)^{4} \cdot \mathbf{E} e^{-4 \theta \psi_{k}}} \\
& \leqslant 4 \sqrt{105}\left[(\mu \Delta t)^{4}+\sigma^{4}(\Delta t)^{4 H}\right] \sqrt{\mathbf{E} e^{-4 \theta \psi_{k}}}
\end{aligned}
$$

since $\psi_{k}$ are Gaussian and therefore $\mathbf{E} \psi_{k}^{8}=105\left(\mathbf{E} \psi_{k}^{2}\right)^{4}$. Further,

$$
\mathbf{E} e^{-4 \theta \psi_{k}} \leqslant \mathbf{E} e^{4\left|\psi_{k}\right|} \leqslant e^{4|\mu| \Delta t} \mathbf{E} e^{4\left|\sigma \Delta B_{k}^{H}\right|} \leqslant 2 e^{4|\mu|+8 \sigma^{2}},
$$

which yields that

$$
\begin{aligned}
\mathbf{E}\left(\xi_{1}(k)\right)^{2} & \leqslant 4 \sqrt{210}\left[(\mu \Delta t)^{4}+\sigma^{4}(\Delta t)^{4 H}\right] e^{2|\mu|+4 \sigma^{2}} \\
& =4 \sqrt{210}(\Delta t)^{2}\left[\mu^{4}(\Delta t)^{2}+\sigma^{4}(\Delta t)^{2(2 H-1)}\right] e^{2|\mu|+4 \sigma^{2}} .
\end{aligned}
$$

Since $2 H-1>0$, we get that $\mathbf{E}\left(\xi_{1}(k)\right)^{2}=\mathcal{O}(\Delta t)^{2}$. Similarly, $\mathbf{E}\left(\xi_{2}(k)\right)^{2}=\mathcal{O}(\Delta t)^{2}$ and according to Lemma 1

$$
\begin{aligned}
& \mathbf{E}\left|\frac{\left|Z_{1}(k)+\xi_{1}(k)+Z_{2}(k)+\xi_{2}(k)\right|}{\left|Z_{1}(k)+\xi_{1}(k)\right|+\left|Z_{2}(k)+\xi_{2}(k)\right|}-\frac{\left|Z_{1}(k)+Z_{2}(k)\right|}{\left|Z_{1}(k)\right|+\left|Z_{2}(k)\right|}\right|= \\
& =\mathbf{E}\left|\frac{\left|\Delta X_{k}+\Delta X_{k+1}\right|}{\left|\Delta X_{k}\right|+\left|\Delta X_{k+1}\right|}-\frac{\left|\Delta B_{k}^{H}+\Delta B_{k+1}^{H}\right|}{\left|\Delta B_{k}^{H}\right|+\left|\Delta B_{k+1}^{H}\right|}\right|=\mathcal{O}\left(2^{-n}\right)^{2 / 3} .
\end{aligned}
$$

Then

$$
\begin{aligned}
& \mathbf{E}\left|R^{1, n}(X)-R^{1, n}\left(B^{H}\right)\right| \\
& \quad \leqslant \frac{1}{n-1} \sum_{k=0}^{n-2} \mathbf{E}\left|\frac{\left|\Delta X_{k}+\Delta X_{k+1}\right|}{\left|\Delta X_{k}\right|+\left|\Delta X_{k+1}\right|}-\frac{\left|\Delta B_{k}^{H}+\Delta B_{k+1}^{H}\right|}{\left|B_{k}^{H}\right|+\left|\Delta B_{k+1}^{H}\right|}\right|=\mathcal{O}\left(2^{-n}\right)^{2 / 3}
\end{aligned}
$$

and, consequently, $R^{1, n}(X) \stackrel{\mathbf{P}}{\longrightarrow} R^{1, n}\left(B^{H}\right)$ as $n \rightarrow \infty$. 
Let $\zeta_{n}:=R^{1, n}(X)-R^{1, n}\left(B^{H}\right)$. Then the Chebyshev's inequality yields

$$
\mathbf{P}\left(\left|\zeta_{n}\right|>2^{-n / 3}\right) \leqslant 2^{n / 3} \mathbf{E}\left|\zeta_{n}\right| \leqslant 2^{-n / 3}
$$

and

$$
\sum_{n=1}^{\infty} \mathbf{P}\left(\left|\zeta_{n}\right|>2^{-n / 3}\right) \leqslant \sum_{n=1}^{\infty} 2^{-n / 3}<\infty
$$

According to the Borel-Cantelli lemma,

$$
\mathbf{P}\left(\limsup _{n \rightarrow \infty}\left\{\left|\zeta_{n}\right|>2^{-n / 3}\right\}\right)=0
$$

which implies that $R^{1, n}(X) \stackrel{\text { a.s. }}{\rightarrow} R^{1, n}\left(B^{H}\right), n \rightarrow \infty$.

The convergence $R^{p, n}\left(B^{H}\right) \stackrel{\text { a.s. }}{\rightarrow} \Lambda_{1}(H), n \rightarrow \infty$ is established in Bardet, Surgailis (2010) and holds for $H \in(0,7 / 8)$ when $p=1$ and $H \in(0,1)$ when $p=2$. Clearly, provided $R^{1, n}(X) \stackrel{\text { a.s. }}{\rightarrow} R^{1, n}\left(B^{H}\right)$ and $R^{p, n}\left(B^{H}\right) \stackrel{\text { a.s. }}{\rightarrow} \Lambda_{1}(H)$, $n \rightarrow \infty$ it follows that $R^{1, n}(X) \stackrel{\text { a.s. }}{\rightarrow} \Lambda_{1}(H), n \rightarrow \infty$ which completes the proof for the case $p=1$. The proof for $p=2$ follows analogously.

Table 2.1. Mean squared errors $\cdot 10^{2}$.

\begin{tabular}{clccc}
\hline Nsp & $n$ & 100 & 500 & 1000 \\
\hline 10 & $B^{H}$ & 1.0997 & 0.6383 & 0.3592 \\
& $X$ & 1.1000 & 0.5891 & 0.3916 \\
50 & $B^{H}$ & 0.5043 & 0.2077 & 0.1537 \\
& $X$ & 0.5817 & 0.2300 & 0.1598 \\
100 & $B^{H}$ & 0.3421 & 0.1781 & 0.1246 \\
& $X$ & 0.3398 & 0.1682 & 0.1257 \\
\hline
\end{tabular}

Fig. 2.2 presents the graph of $\Lambda_{2}(H)$ together with the graph of $R^{2,100}(X)$ averaged over 50 sample paths, $X$ being the fractional geometric Brownian motion. Table 2.1 shows the comparison of mean squared errors of $R^{2, n}(X)-$ $\Lambda_{2}(H)$ and $R^{2, n}\left(B^{H}\right)-\Lambda_{2}(H)$ for the sample path lengths $n=100,200,500$ and the numbers of sample paths $N s p=20,50,100$. The coefficients of the fractional geometric Brownian motion were chosen as $X_{0}=1, \mu=-0.3$, $\sigma=0.5$. 


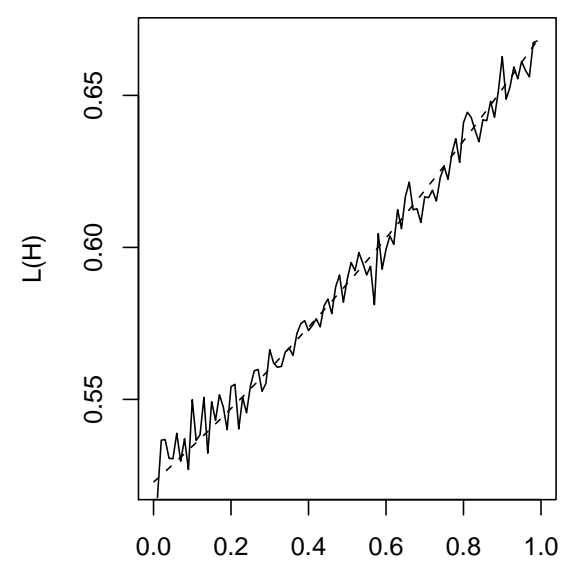

Fig. 2.2. Graphs of $\Lambda_{2}(H)$ and $R^{2,100}(X)$

\subsection{The convergence rate of the Gladyshev estimator}

In this section we define the modified Gladyshev's estimator of the fBm parameter $H$ and derive the rate of convergence of it to its real value. To our knowledge, this problem is new and interesting from the practical point of view.

To recall, for a real-valued process $X=\left\{X_{t} ; t \in[0,1]\right\}$ taking values at the points $t_{k}^{n}, k=0, \ldots, N_{n}$, the first order quadratic variation is defined as

$$
V_{n}^{(1)}(X, 2)=\sum_{k=1}^{N_{n}}\left(\Delta X_{k}^{n}\right)^{2}, \quad \Delta X_{k}^{n}=X\left(t_{k}^{n}\right)-X\left(t_{k-1}^{n}\right) .
$$

Let $B^{H}$ be the fractional Brownian motion with the Hurst index $H$. Set $t_{k}^{n}=k 2^{-n}, k=1, \ldots, 2^{n}$. It is known (see Gladyshev (1963)) that

$$
2^{n(2 H-1)} V_{n}^{(1)}\left(B^{H}, 2\right) \stackrel{\text { a.s. }}{\longrightarrow} 1 \text { as } n \rightarrow \infty .
$$

This result yields that

$$
\widetilde{H}_{n}=\frac{1}{2}-\frac{\ln V_{n}^{(1)}\left(B^{H}, 2\right)}{2 n \ln 2}
$$

is a strongly consistent estimator of $H$. 


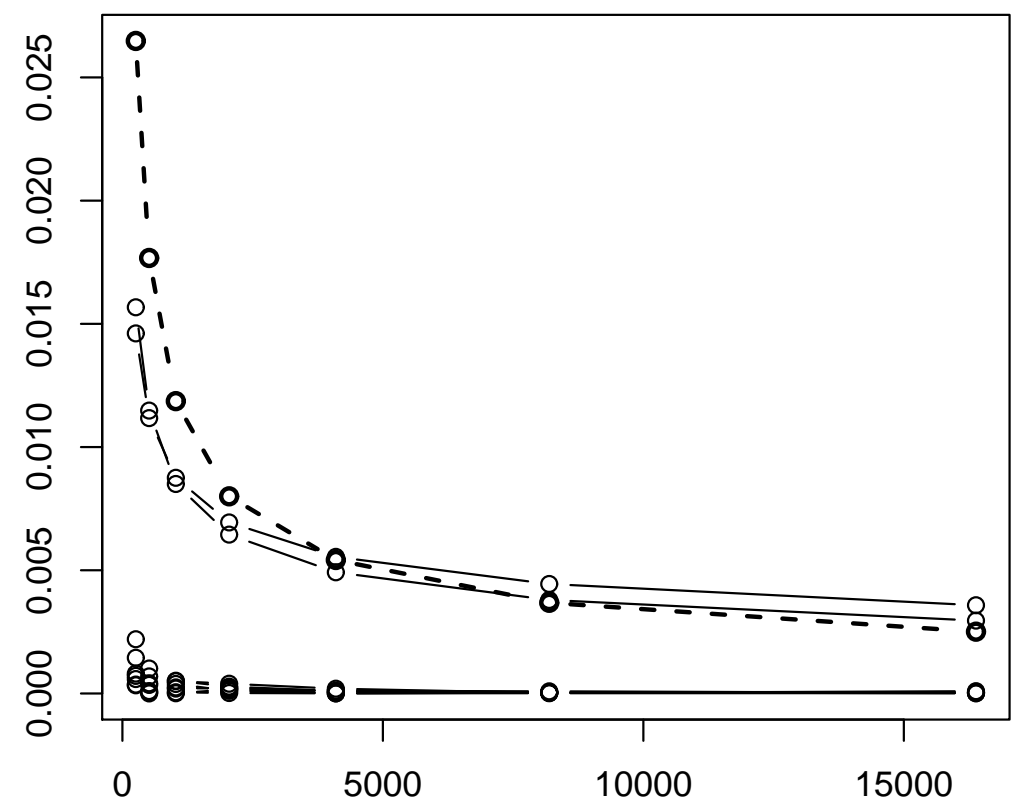

Fig. 2.3. Graphs of $\sqrt{N_{n}^{-1} \ln N_{n}}$ and $\mathbf{E}\left|\widehat{H}_{n}-H\right|, H \in\{0.55,0.60, \ldots, 0.95\}$

Let us define a modified Gladyshev's estimator of the Hurst index $H$ by

$$
\widehat{H}_{n}=\left(\frac{1}{2}-\frac{\ln \left[V_{n}^{(1)}\left(B^{H}, 2\right)\right]}{2 \ln N_{n}}\right) \mathbf{1}_{C_{n}},
$$

for a regular subdivision $\pi_{n}$, where

$$
C_{n}=\left\{V_{n}^{(1)}\left(B^{H}, 2\right) \geqslant N_{n}^{-2}\right\} .
$$

The estimate $\widehat{H}_{n}$ is strongly consistent. Moreover, we can derive the rate of convergence of it to $H$. This follows from the next theorem. This result is illustrated by Fig. 2.3 which presents the graph of $\sqrt{N_{n}^{-1} \ln N_{n}}$ (the dotted line) and those of $\mathbf{E}\left|\widehat{H}_{n}-H\right|$ for $H \in(0.55,0.60, \ldots, 0.95)$ against the sample path lengths $N_{n} \in\left\{2^{k}+1 ; k=8, \ldots, 14\right\}$. It can be seen that the convergence rate is determined by the behavior of $\widehat{H}_{n}$ for the values of $H$ which are close to 1 , as the lines closest to the dotted line correspond to $H=$ 0.9 and $H=0.95$.

2.11 Theorem. Let $B^{H}, 1 / 2<H<1$, be the fractional Brownian motion. $\widehat{H}_{n}$ is a strongly consistent estimator of the Hurst index $H$ and the following 
rates of convergence hold:

$$
\left|\widehat{H}_{n}-H\right|=\mathcal{O}\left(\sqrt{N_{n}^{-1} \ln N_{n}}\right) \quad \text { a.s. if } \sum_{n=1}^{\infty} N_{n}^{-2}<\infty
$$

and

$$
\mathbf{E}\left|\widehat{H}_{n}-H\right|=\mathcal{O}\left(\sqrt{N_{n}^{-1} \ln N_{n}}\right)
$$

Proof of Theorem 2.11. First we have

$$
\widehat{H}_{n}=H \mathbf{1}_{C_{n}}-\frac{\ln B_{n}}{2 \ln N_{n}} \mathbf{1}_{C_{n}},
$$

where $B_{n}=N_{n}^{2 H-1} V_{n}^{(1)}\left(B^{H}, 2\right)$. Thus

$$
\begin{aligned}
\left|\widehat{H}_{n}-H\right| & \leqslant H \mathbf{1}_{\bar{C}_{n}}+\left|\frac{\ln B_{n}}{2 \ln N_{n}}\right| \mathbf{1}_{\left\{B_{n} \geqslant N_{n}^{-2}\right\}} \\
& \leqslant H \mathbf{1}_{\left\{B_{n}<N_{n}^{-1}\right\}}-\frac{\ln B_{n}}{2 \ln N_{n}} \mathbf{1}_{\left\{N_{n}^{-2} \leqslant B_{n}<1\right\}}+\frac{\ln B_{n}}{2 \ln N_{n}} \mathbf{1}_{\left\{B_{n} \geqslant 1\right\}} .
\end{aligned}
$$

Let $\left(\delta_{n}\right)$ be a sequence of positive numbers such that $\delta_{n}<1$ and $\delta_{n} \downarrow 0$. The inequality $-\ln (1-x) \leqslant 20 x, 0 \leqslant x \leqslant 19 / 20$, yields

$$
\begin{aligned}
\left(-\ln B_{n}\right) \mathbf{1}_{\left\{1-\delta_{n} \leqslant B_{n}<1\right\}} & =\left(-\ln \left[1-\left(1-B_{n}\right)\right]\right) \mathbf{1}_{\left\{1-\delta_{n} \leqslant B_{n}<1\right\}} \\
& \leqslant 20\left(1-B_{n}\right) \mathbf{1}_{\left\{1-\delta_{n} \leqslant B_{n}<1\right\}},
\end{aligned}
$$

if $\delta_{n} \leqslant 19 / 20$. So, it follows that

$$
\begin{aligned}
-\frac{\ln B_{n}}{2 \ln N_{n}} \mathbf{1}_{\left\{N_{n}^{-2} \leqslant B_{n}<1\right\}} & \leqslant \mathbf{1}_{\left\{N_{n}^{-2} \leqslant B_{n}<1-\delta_{n}\right\}}+10 \frac{1-B_{n}}{\ln N_{n}} \mathbf{1}_{\left\{1-\delta_{n} \leqslant B_{n}<1\right\}} \\
& \leqslant \mathbf{1}_{\left\{N_{n}^{-2} \leqslant B_{n}<1-\delta_{n}\right\}}+\frac{10 \delta_{n}}{\ln N_{n}} \mathbf{1}_{\left\{1-\delta_{n} \leqslant B_{n}<1\right\}} .
\end{aligned}
$$

The inequality $\ln (1+x) \leqslant x, x \geqslant 0$, yields

$$
\left(\ln B_{n}\right) \mathbf{1}_{\left\{B_{n} \geqslant 1\right\}}=\left(\ln \left[1+\left(B_{n}-1\right)\right]\right) \mathbf{1}_{\left\{B_{n} \geqslant 1\right\}} \leqslant\left(B_{n}-1\right) \mathbf{1}_{\left\{B_{n} \geqslant 1\right\}} .
$$


Thus

$$
\begin{aligned}
\frac{\ln B_{n}}{2 \ln N_{n}} \mathbf{1}_{\left\{B_{n} \geqslant 1\right\}} & \leqslant \frac{B_{n}-1}{2 \ln N_{n}} \mathbf{1}_{\left\{1 \leqslant B_{n} \leqslant 1+\delta_{n}\right\}}+\frac{B_{n}-1}{2 \ln N_{n}} \mathbf{1}_{\left\{B_{n}>1+\delta_{n}\right\}} \\
& \leqslant \frac{\delta_{n}}{2 \ln N_{n}} \mathbf{1}_{\left\{1 \leqslant B_{n} \leqslant 1+\delta_{n}\right\}}+\frac{B_{n}-1}{2 \ln N_{n}} \mathbf{1}_{\left\{B_{n}>1+\delta_{n}\right\}} .
\end{aligned}
$$

Inequalities (2.8) and (2.9) imply that

$$
\left|\widehat{H}_{n}-H\right| \leqslant\left(2+\frac{B_{n}-1}{2 \ln N_{n}}\right) \mathbf{1}_{\left\{\left|B_{n}-1\right|>\delta_{n}\right\}}+\frac{10 \delta_{n}}{\ln N_{n}} .
$$

To complete the proof it suffices to estimate the first term in the inequality (2.10) by using the Hanson and Wright inequality (Hanson, Wright (1971)). Note that $N_{n}^{2 H-1} V_{n}^{(1)}\left(B^{H}, 2\right)$ is the square of the Euclidean norm of an $N_{n^{-}}$ dimensional Gaussian vector $X_{n}$ with components

$$
N_{n}^{2 H-1} \Delta B_{k}^{H, n}, \quad 1 \leqslant k \leqslant N_{n} .
$$

Using a linear transformation of $X_{n}$ one can get a new Gaussian vector $Y_{n}$ with independent components. So there exist nonnegative real numbers $\left\{\lambda_{1, n}, \ldots, \lambda_{N_{n}, N_{n}}\right\}$ and one $N_{n}$-dimensional Gaussian vector $Y_{n}$ such that its components are independent standard Gaussian random variables and

$$
N_{n}^{2 H-1} V_{n}^{(1)}\left(B^{H}, 2\right)=\sum_{j=1}^{N_{n}} \lambda_{j, N_{n}}\left(Y_{n}^{(j)}\right)^{2} .
$$

Numbers $\left\{\lambda_{1, n}, \ldots, \lambda_{N_{n}, N_{n}}\right\}$ are the eigenvalues of the symmetric $N_{n} \times N_{n^{-}}$ matrix

$$
\left(N_{n}^{2 H-1} \mathbf{E}\left[\Delta B_{j}^{H, n} \Delta B_{k}^{H, n}\right]\right)_{1 \leqslant j, k \leqslant N_{n}} .
$$

With the arguments of Gine, Klein (1975) and Bégyn (2005) one can get the inequality

$$
\mathbf{P}\left(N_{n}^{2 H-1}\left|V_{n}^{(1)}\left(B^{H}, 2\right)-\mathbf{E} V_{n}^{(1)}\left(B^{H}, 2\right)\right| \geqslant \varepsilon\right) \leqslant 2 \exp \left(-K \varepsilon^{2} N_{n}\right),
$$

which follows directly from the Hanson and Wright inequality, where $0<\varepsilon \leqslant 1$ and $K$ is a positive constant. Set

$$
\delta_{n}^{2}=\frac{2 \ln N_{n}}{K N_{n}} .
$$


From the inequality (2.11) it follows that

$$
\mathbf{P}\left(\left|B_{n}-1\right|>\delta_{n}\right) \leqslant \frac{2}{N_{n}^{2}} .
$$

Obviously,

$$
\mathbf{P}\left(\left(2+\frac{B_{n}-1}{2 \ln N_{n}}\right) \mathbf{1}_{\left\{\left|B_{n}-1\right|>\delta_{n}\right\}}>0\right) \leqslant \mathbf{P}\left(\left|B_{n}-1\right|>\delta_{n}\right) \leqslant \frac{2}{N_{n}^{2}} .
$$

Under the conditions of the theorem, the Borel-Cantelli lemma yields

$$
\mathbf{P}\left(\limsup _{n \rightarrow \infty}\left\{\left(\frac{1}{2}+\frac{B_{n}-1}{2 \ln N_{n}}\right) \mathbf{1}_{\left\{\left|B_{n}-1\right|>\delta_{n}\right\}}>0\right\}\right)=0,
$$

i. e.,

$$
\left(2+\frac{B_{n}-1}{2 \ln N_{n}}\right) \mathbf{1}_{\left\{\left|B_{n}-1\right|>\delta_{n}\right\}}=0
$$

for sufficiently large $n$. From the above results and the inequality (2.10) it follows that

$$
\left|\widehat{H}_{n}-H\right|=\mathcal{O}\left(\sqrt{N_{n}^{-1} \ln N_{n}}\right) \quad \text { a.s. }
$$

which completes the proof of (2.6). Note that from the inequalities (2.10) and (2.12) we get

$$
\mathbf{E}\left|\widehat{H}_{n}-H\right| \leqslant \frac{2}{N_{n}^{2}}+\mathbf{E} \frac{\left|B_{n}-1\right|}{2 \ln N_{n}} \mathbf{1}_{\left\{\left|B_{n}-1\right|>\delta_{n}\right\}}+\frac{10 \delta_{n}}{\ln N_{n}} .
$$

It remaints to estimate the second term on the right side of the previous inequality. Note that

$$
\begin{aligned}
& \mathbf{E}\left|B_{n}-1\right| \mathbf{1}_{\left\{\left|B_{n}-1\right|>\delta_{n}\right\}} \\
& \leqslant \mathbf{E}^{1 / 2}\left|B_{n}-1\right|^{2} \sqrt{\mathbf{P}\left(\left|B_{n}-1\right|>\delta_{n}\right)} \leqslant \frac{2}{N_{n}} \mathbf{E}^{1 / 2}\left(B_{n}^{2}+1\right) \\
& \leqslant \frac{2}{N_{n}}\left(N_{n}^{2 H-1 / 2} \mathbf{E}^{1 / 2} \sum_{k=1}^{N_{n}}\left|\Delta B_{k}^{H, n}\right|^{4}+1\right) \\
& \leqslant \frac{2}{N_{n}}\left(\sqrt{3} N_{n}^{-1 / 2}+1\right) .
\end{aligned}
$$


Thus

$$
\mathbf{E}\left|\widehat{H}_{n}-H\right| \leqslant \frac{2}{N_{n}^{2}}+\frac{\sqrt{3} N_{n}^{-1 / 2}+1}{N_{n} \ln N_{n}}+\frac{10 \delta_{n}}{\ln N_{n}},
$$

which concludes the proof of (2.7).

\subsection{Conclusions of the second chapter}

1. Having proved the Theorem 1 and Theorem 3, the asymptotics of quadratic variations of the solution of the stochastic differential equation (1.1) in case of regularly spaced observations of the process were obtained. It was shown in Theorem 2 and Theorem 4 that the estimators of the Hurst index $H$ originally obtained by Istas, Lang (1997) and Benassi et al (1998) for the fractional Brownian motion remain strongly consistent when the underlying process is the solution of the stochastic integral equation which is not necessarily Gaussian.

2. Having proved the Theorem 6 and Theorem 8 , the asymptotics of quadratic variations of the solution of the stochastic differential equation (1.1) in case of irregularly spaced observations of the process were derived. It was shown in Theorem 7 that $\widetilde{H}_{d v 1}^{n}$, the proposed estimator of the Hurst index $H$ based on the first order quadratic variations is strongly consistent in case of irregularly spaced observations.

3. In Theorem 9 it was proved that the obtained estimators remain strongly consistent if the solution of the stochastic differential equation is replaced with its Milstein approximation.

4. In Theorem 10 it was proved that the increment ratio statistic can be applied to estimate the Hurst index $H$ of the fractional geometric Brownian motion.

5. In Theorem 11 the rate of convergence of the modified Gladyshev estimator of the Hurst index $H$ to its real value was derived. 



\section{Modelling of the estimators}

The goal of this chapter is to compare the behavior of the estimators based on quadratic variations with some of the other known estimators, namely the naive and ordinary least squares Gladyshev and $\eta$-summing oscillation estimators, the variogram estimator and the IR estimator. These estimators are described in Section 3.2. Most of them were examined for Gaussian processes. The models chosen for comparison of these estimators were the fractional Ornstein-Uhlenbeck $(\mathrm{O}-\mathrm{U})$ process and the fractional geometric Brownian motion $(\mathrm{gBm})$. The initial inference about the behavior of these estimators was drawn for the O-U process which is Gaussian, while the $\mathrm{gBm}$ process was used to check how the estimators behave in a non-Gaussian case.

In order to achieve that, a sufficient amount of sample paths of $\mathrm{fBm}$ is required. These sample paths were generated using the circulant matrix embedding method as described in Coeurjolly (2000) and the references therein. Let $n$ denote the length of the sample path. The circulant matrix embedding method uses a fast Fourier transform which bypasses the matrix computations and therefore is sufficiently fast even for large values of $n .100$ sample paths of the length $n=2^{14}+1$ were generated for each value of $H \in$ $\{0.55,0.6, \ldots, 0.95\}$ on the unit interval $t \in[0,1]$.

The next step would be to use the generated $\mathrm{fBm}$ data to construct the sample paths of the considered processes. However, it's not always possible to find and use the explicit solution of the considered stochastic differential 
equation, therefore this solution might need to be replaced with its time discrete approximation. For a process $X_{t}$, its Milstein approximation at points $t_{k}^{n}, k=$ $1, \ldots, n$ is defined as

$X_{k}^{n}=X_{k-1}^{n}+f\left(X_{k-1}^{n}\right) \Delta t_{k}+g\left(X_{k-1}^{n}\right) \Delta B_{k}^{H}+\frac{1}{2} g\left(X_{k-1}^{n}\right) g^{\prime}\left(X_{k-1}^{n}\right)\left(\Delta B_{k}^{H}\right)^{2}$,

where $g^{\prime}$ denotes the derivative of $g$. The fractional Ornstein-Uhlenbeck (O-U) and the fractional geometric Brownian motion $(\mathrm{gBm})$ processes are defined as

$d X_{t}=-\mu X_{t} d t+\sigma d B_{t}^{H}, \quad X_{0}=c$,

$d X_{t}=\mu X_{t} d t+\sigma X_{t} d B_{t}^{H}, \quad X_{0}=c$.

The solutions of these equations are, respectively,

$$
X_{t}=e^{-\mu t}\left(c+\sigma \int_{0}^{t} e^{\mu s} d B_{s}^{H}\right) \quad \text { and } \quad X_{t}=c \exp \left(\mu t+\sigma B_{t}^{H}\right) .
$$

In fact, for the O-U process the Milstein approximation is reduced to the Euler one due to $g^{\prime}\left(X_{k-1}^{n}\right)=(\sigma)^{\prime}=0$. The constants were chosen as $c=1, \mu=$ $0.5, \sigma=0.7$ in the $\mathrm{O}-\mathrm{U}$ case and $c=1, \mu=0.2, \sigma=0.5$ in the $\mathrm{gBm}$ case. The error introduced by using these approximated sample paths was negligible compared to the errors of the estimators themselves and will be ignored further on. All computations were performed using the $\mathrm{R}$ software environment ( $\mathrm{R}$ Development Core Team (2009)).

\subsection{Generation of the fractional Brownian motion}

The algorithm to generate one sample path of the length $n$, using the circulant matrix embedding method, is as follows:

- Choose $M=2^{p} \geqslant 2(n-1)$. Define the $M$-vector

$$
\begin{aligned}
V=(r(0), r(1), \ldots, & ,\left(\frac{M}{2}-1\right), r\left(\frac{M}{2}\right), \\
& \left., r\left(\frac{M}{2}-1\right), \ldots, r(2), r(1)\right),
\end{aligned}
$$


where

$$
r(k)=\frac{1}{2 n^{2 H}}\left[|k+1|^{2 H}-2 k^{2 H}+|k-1|^{2 H}\right]
$$

is the autocovariance function of the fractional Gaussian noise.

- Compute $W=\left(w_{1}, \ldots, w_{M}\right)$, the fast Fourier transformation of $V$. All the coordinates of $W$ must be non-negative. If this is not the case, the value of $p$ must be increased until this requirement is met.

- Generate $U_{j}, V_{j} \sim \mathcal{N}(0,1)$ for all $1 \leqslant j<\frac{M}{2}$ and let $Z_{1}=U_{1}$, $Z_{\frac{M}{2}+1}=V_{1}$,

$$
Z_{j}=\frac{1}{\sqrt{2}}\left(U_{j}+i V_{j}\right), \quad Z_{M+2-j}=\frac{1}{\sqrt{2}}\left(U_{j}-i V_{j}\right), \quad 1<j \leqslant \frac{M}{2} .
$$

Then, define the $M$-vector $U$ as

$$
U_{k}=\sqrt{w_{k}} Z_{k}, \quad k=1, \ldots, M .
$$

- Compute $Y$ as an inverse fast Fourier transformation of the complex vector $U$ and define $X$ as

$$
X_{k}=X_{k-1}+\operatorname{Re}\left(Y_{k}\right), \quad X_{0}=0, \quad k=1, \ldots, n-1,
$$

$\operatorname{Re}(Y)$ denoting the real part of the complex variable $Y$.

The obtained vector $X$ is the desired sample path of the fractional Brownian motion with the Hurst index $H$.

\subsection{Estimators}

\subsubsection{Discrete variation estimators}

For a real-valued process $X=\left\{X_{t} ; t \in[0,1]\right\}$, we define the first and second order quadratic variations as

$$
V_{n}^{(1)}(X, 2)=\sum_{k=1}^{n}\left(\Delta_{k}^{(1)} X\right)^{2}, \quad V_{n}^{(2)}(X, 2)=\sum_{k=1}^{n-1}\left(\Delta_{k}^{(2)} X\right)^{2},
$$


where

$$
\Delta_{k}^{(1)} X=X\left(t_{k}^{n}\right)-X\left(t_{k-1}^{n}\right), \quad \Delta_{k}^{(2)} X=X\left(t_{k+1}^{n}\right)-2 X\left(t_{k}^{n}\right)+X\left(t_{k-1}^{n}\right) .
$$

and $t_{k}^{n}=\frac{k}{n}$. Let $X$ be the solution of (1.1). It is known (see Kubilius, Melichov (2008) - Kubilius, Melichov (2010)) that

$$
\widehat{H}_{d v 1}^{n}=\frac{1}{2}-\frac{1}{2 \ln 2} \ln \frac{V_{2 n}^{(1)}(X, 2)}{V_{n}^{(1)}(X, 2)}, \quad \widehat{H}_{d v 2}^{n}=\frac{1}{2}-\frac{1}{2 \ln 2} \ln \frac{V_{2 n}^{(2)}(X, 2)}{V_{n}^{(2)}(X, 2)}
$$

are strongly consistent estimators of the Hurst index $H$, i.e.,

$$
\widehat{H}_{d v 1}^{n}-H \stackrel{\text { a.s. }}{\longrightarrow} 0 \quad \text { and } \quad \widehat{H}_{d v 2}^{n}-H \stackrel{\text { a.s. }}{\longrightarrow} 0 \quad \text { as } \quad n \rightarrow \infty .
$$

Here $V_{2 n}^{(\cdot)}(X, 2)$ corresponds to the quadratic variation of the whole sample path while $V_{n}^{(\cdot)}(X, 2)$ is the variation of the subset $\left\{X_{k}: k=2 j, 0 \leqslant j \leqslant\right.$ $[n / 2]\},[x]$ denotes the integer part of $x$.

\subsubsection{Gladyshev and $\eta$-summing oscillation estimators}

The following estimators were described in R. Norvaiša and D.M. Salopek (2002, Norvaiša, Salopek (2002)). The ordinary least squares (OLS) Gladyshev and $\eta$-summing oscillation estimators require a sample path of the length $2^{n}+$ $1, n \in \mathbb{N}$, which dictated the length of our modeled sample paths. Define $\eta_{M}=\left\{N_{m}=2^{m}: 1 \leqslant m \leqslant M\right\}$ and let

$$
s(m)=\sum_{i=1}^{N_{m}}\left[X\left(\frac{i}{N_{m}}\right)-X\left(\frac{i-1}{N_{m}}\right)\right]^{2} .
$$

The naive Gladyshev estimator of the Hurst index $\mathrm{H}$ is given by

$$
\widehat{H}_{g n}^{M}=\frac{\log \sqrt{s(M) 2^{-M}}}{\log 2^{-M}},
$$

and the OLS Gladyshev estimator is given by

$$
\widehat{H}_{g o}^{M}=\frac{\sum_{m=1}^{M}\left(z_{m}-\bar{z}\right)^{2}}{\sum_{m=1}^{M}\left(z_{m}-\bar{z}\right) m}
$$

where $z_{m}=\log _{2} \sqrt{2^{m} / s(m)}$ for $m \in\{1, \ldots, M\}$ and $\bar{z}=M^{-1} \sum_{m=1}^{M} z_{m}$. 
For each $m \in\{1, \ldots, M\}$, define

$$
Q(m)=\sum_{i=1}^{N_{m}}\left[\max _{t_{k}^{n} \in \Delta_{i, m}}\left\{X\left(t_{k}^{n}\right)\right\}-\min _{t_{k}^{n} \in \Delta_{i, m}}\left\{X\left(t_{k}^{n}\right)\right\}\right],
$$

where

$$
\Delta_{i, m}=\left[\frac{i-1}{N_{m}}, \frac{i}{N_{m}}\right] .
$$

The naive oscillation estimator is defined by

$$
\widehat{H}_{o s n}^{M}=\frac{\log _{2}\left(N_{M} / Q(M)\right)}{\log _{2} N_{M}}
$$

and the OLS oscillation estimator is defined by

$$
\widehat{H}_{\text {oso }}^{M}=\frac{\sum_{m=1}^{M}\left(z_{m}-\bar{z}\right)^{2}}{\sum_{m=1}^{M}\left(z_{m}-\bar{z}\right) N_{m}}
$$

where $z_{m}=\log _{2} \sqrt{N_{m} / Q(m)}$ and $\bar{z}=M^{-1} \sum_{m=1}^{M} z_{m}$.

For $M=14$ we simulate estimates defined above.

\subsubsection{Variogram estimator}

The variogram of the process $X=\left\{X_{t}, t \in[0,1]\right\}$ for the lag $\ell$ is defined Chronopoulou, Viens (2010) as

$$
V(\ell)=\mathbb{E}\left[\left(X_{t}-X_{t-\ell}\right)^{2}\right] .
$$

In order to estimate the Hurst index $H$, we choose a set of lags, in our case, it was $\left\{\ell=2^{i} ; i=0, \ldots, 5\right\}$. Then $\widehat{H}_{v a r}^{n}=b / 2$, where $b$ is the slope of the linear regression line of $\log (V(\ell))$ against $\log (\ell)$.

\subsubsection{Increment ratios estimator}

This estimator was proposed by Bardet, Surgailis (2010). For the O-U or $\mathrm{gBm}$ process $X=\left\{X_{t} ; t \in[0,1]\right\}$ given at points $t_{k}^{n}=k / n, k=$ $0,1, \ldots, n$, the increment ratios (IR) estimator of $H$ can be computed using 
the approximated formula

$$
\widehat{H}_{i r}^{n}=\frac{1}{0.1468}\left(\frac{1}{n-2} \sum_{k=1}^{n-2} \frac{\left|\Delta_{k}^{(2)} X+\Delta_{k+1}^{(2)} X\right|}{\left|\Delta_{k}^{(2)} X\right|+\left|\Delta_{k+1}^{(2)} X\right|}-0.5174\right),
$$

where $\Delta_{k}^{(2)} X=X\left(t_{k+1}^{n}\right)-2 X\left(t_{k}^{n}\right)+X\left(t_{k-1}^{n}\right)$.

\subsection{The Ornstein-Uhlenbeck process}

\subsubsection{Dependance on the value of the Hurst index}

The first goal of this section is to compare the behavior of these estimators for different values of the Hurst index $H$. Table 3.1 presents the biases $\bar{H}-H=\mathbf{E}(\widehat{H}-H)$ as well as the mean squared errors defined as $M S E(\widehat{H})=\mathbf{E}(\widehat{H}-H)^{2}$ for the sample path lengths of, respectively, $2^{14}+1$ and $2^{10}+1$ points. Figure B.1 (see Appendix) illustrates this further presenting the boxplots of the considered estimators for the length of sample paths $n=2^{14}+1$ points. Here and further in this section the figures related to the estimators $\widehat{H}_{g n}$ and $\widehat{H}_{g o}$ are omitted, since their behavior does not significantly differ from the behavior of $\widehat{H}_{o s n}$ and $\widehat{H}_{\text {oso }}$. The numbers printed in bold correspond to the estimators that performed better than the others for the specific value of $H$ and the considered numeric characteristic.

It can be seen that the estimators $\widehat{H}_{d v 1}, \widehat{H}_{v a r}, \widehat{H}_{g n}$ and $\widehat{H}_{o s n}$ exhibit increases of the biases and the mean squared errors for larger values of $H . \widehat{H}_{g o}$ and $\widehat{H}_{\text {oso }}$ seem to be less dependant on that, however, they tend to slightly undervalue the Hurst index when it is close to $1 . \widehat{H}_{i r}$ tends to slightly undervalue $H$ when $H<3 / 4$ and to overvalue it when $H>3 / 4$; the most likely cause of this is the numeric constants in the formula used for this estimator. The behavior of $\widehat{H}_{d v 2}$ does not change noticeably for different values of $H$.

Another interesting observation is the, comparatively, very low mean squared errors of $\widehat{H}_{g n}$ and $\widehat{H}_{o s n}$ which they display as long as the Hurst index is not too close to 1 . However these estimators also possess the largest bias. $\widehat{H}_{g o}$ and $\widehat{H}_{\text {oso }}$, the OLS versions of these two estimators behave in a completely different way - they have smaller biases which are comparable to those of the other considered estimators, but this comes at the cost of heavily increased MSE. 
Table 3.1. Comparison of the estimators for the O-U process.

\begin{tabular}{|c|ccccc|}
\hline \multicolumn{1}{|c}{$H$} & & 0.55 & 0.7 & 0.8 & 0.95 \\
\hline \hline \multirow{5}{*}{$M S E$} & dv1 & 0.008 & $\mathbf{0 . 0 0 5}$ & $\mathbf{0 . 0 0 8}$ & $\mathbf{0 . 0 2 0}$ \\
& dv2 & 0.015 & 0.011 & 0.011 & $\mathbf{0 . 0 1 0}$ \\
& var & $\mathbf{0 . 0 0 7}$ & 0.009 & 0.014 & 0.024 \\
& gn & $\mathbf{0 . 0 0 2}$ & $\mathbf{0 . 0 0 2}$ & $\mathbf{0 . 0 0 3}$ & $\mathbf{0 . 0 2 1}$ \\
& osn & $\mathbf{0 . 0 0 4}$ & $\mathbf{0 . 0 0 4}$ & $\mathbf{0 . 0 0 5}$ & 0.025 \\
& go & 0.038 & 0.037 & 0.048 & 0.050 \\
& oso & 0.041 & 0.037 & 0.050 & 0.053 \\
& ir & 0.022 & 0.019 & 0.019 & 0.022 \\
\hline \hline \multirow{5}{*}{$-H$} & dv1 & $\mathbf{0 . 0 0 0}$ & $\mathbf{0 . 0 0 1}$ & $\mathbf{- 0 . 0 0 1}$ & $\mathbf{- 0 . 0 0 9}$ \\
& dv2 & $\mathbf{0 . 0 0 0}$ & $\mathbf{0 . 0 0 1}$ & -0.002 & $\mathbf{- 0 . 0 0 1}$ \\
& var & $\mathbf{0 . 0 0 0}$ & $-\mathbf{0 . 0 0 1}$ & $\mathbf{0 . 0 0 0}$ & $\mathbf{- 0 . 0 1 2}$ \\
& gn & 0.037 & 0.037 & 0.037 & 0.041 \\
& osn & 0.060 & 0.060 & 0.060 & 0.063 \\
& go & -0.013 & -0.024 & -0.019 & -0.032 \\
& oso & -0.004 & -0.017 & -0.009 & -0.020 \\
& ir & -0.019 & -0.009 & $\mathbf{- 0 . 0 0 1}$ & 0.028 \\
\hline
\end{tabular}

(a) $N=2^{14}+1$

\begin{tabular}{|ccccc|}
\hline$H$ & 0.55 & 0.7 & 0.8 & 0.95 \\
\hline \hline dv1 & 0.027 & 0.024 & $\mathbf{0 . 0 2 6}$ & $\mathbf{0 . 0 3 0}$ \\
dv2 & 0.050 & 0.054 & 0.050 & 0.044 \\
var & 0.029 & 0.031 & 0.038 & $\mathbf{0 . 0 4 0}$ \\
gn & $\mathbf{0 . 0 0 6}$ & $\mathbf{0 . 0 0 6}$ & $\mathbf{0 . 0 1 0}$ & $\mathbf{0 . 0 4 0}$ \\
osn & $\mathbf{0 . 0 1 0}$ & $\mathbf{0 . 0 1 1}$ & $\mathbf{0 . 0 1 5}$ & 0.048 \\
go & 0.092 & 0.061 & 0.071 & 0.068 \\
oso & 0.093 & 0.060 & 0.073 & 0.071 \\
ir & 0.074 & 0.067 & 0.069 & 0.077 \\
\hline dv1 & $\mathbf{0 . 0 0 0}$ & $\mathbf{- 0 . 0 0 4}$ & $\mathbf{- 0 . 0 0 1}$ & $\mathbf{- 0 . 0 1 6}$ \\
dv2 & $\mathbf{0 . 0 0 1}$ & $\mathbf{0 . 0 0 0}$ & $\mathbf{- 0 . 0 0 2}$ & $\mathbf{0 . 0 0 8}$ \\
var & $\mathbf{0 . 0 0 0}$ & -0.012 & -0.007 & -0.025 \\
gn & 0.051 & 0.052 & 0.052 & 0.062 \\
osn & 0.084 & 0.084 & 0.084 & 0.091 \\
go & -0.010 & -0.035 & -0.030 & -0.044 \\
oso & 0.003 & -0.023 & -0.014 & -0.028 \\
ir & -0.009 & $\mathbf{- 0 . 0 0 4}$ & $\mathbf{- 0 . 0 0 4}$ & 0.039 \\
\hline
\end{tabular}

(b) $N=2^{10}+1$

\subsubsection{Dependance on the length of the sample path}

The second goal of this section is to compare the behavior of these estimators for different lengths of sample paths as well as to illustrate how the estimators' variances fluctuate as the length of the sample paths is increased. Table 3.2 shows the mean squared errors and the biases for the Hurst index values of 0.65 and 0.85 , respectively. Figure B.2 (see Appendix) presents the boxplots of the estimators for $H=0.85$.

The first obvious observation is that the bias of $\widehat{H}_{g n}$ and $\widehat{H}_{o s n}$ increases as the length of the sample paths is decreased. $\widehat{H}_{g o}$ and $\widehat{H}_{\text {oso }}$ do not share this property, however their mean squared errors display only minor decreases when longer sample paths are taken. The other estimators show a rather regular decrease of their mean squared errors which is further illustrated by Figure B.3 (see Appendix) presenting the plots of $\log (S D)$ against $\log (n)$ for $H \in$ $\{0.55,0.6, \ldots, 0.95\}$ where SD denotes the standard deviations.

Figure B. 3 shows the rate at which the standard deviation decreases as the sample path length is increased. It can be seen that this rate depends on the value of $H$ for all the estimators except $\widehat{H}_{d v 2}$ and $\widehat{H}_{i r}$. The general trend is that this rate is lower for higher values of $H$ which is most notable for $\widehat{H}_{g n}$ and $\widehat{H}_{o s n}$. On the other hand $\widehat{H}_{d v 2}$ and $\widehat{H}_{i r}$ display no dependance of this kind. 
Table 3.2. Comparison of the estimators for the O-U process for sample path lengths $N=2^{k}+1$.

\begin{tabular}{|c|ccccc|}
\hline & $k$ & 8 & 10 & 12 & 14 \\
\hline \hline \multirow{5}{*}{$M S E$} & dv1 & $\mathbf{0 . 0 5 3}$ & $\mathbf{0 . 0 2 5}$ & $\mathbf{0 . 0 1 4}$ & $\mathbf{0 . 0 0 7}$ \\
& dv2 & 0.102 & 0.055 & 0.027 & 0.013 \\
& var & 0.067 & 0.033 & 0.018 & 0.009 \\
gn & $\mathbf{0 . 0 1 3}$ & $\mathbf{0 . 0 0 6}$ & $\mathbf{0 . 0 0 3}$ & $\mathbf{0 . 0 0 2}$ \\
& osn & $\mathbf{0 . 0 2 1}$ & $\mathbf{0 . 0 1 1}$ & $\mathbf{0 . 0 0 6}$ & $\mathbf{0 . 0 0 4}$ \\
& go & 0.084 & 0.063 & 0.049 & 0.039 \\
& oso & 0.086 & 0.064 & 0.050 & 0.040 \\
& ir & 0.172 & 0.079 & 0.037 & 0.018 \\
\hline \hline \multirow{5}{*}{$H$} & dv1 & $\mathbf{- 0 . 0 0 5}$ & $\mathbf{0 . 0 0 0}$ & $\mathbf{0 . 0 0 2}$ & $\mathbf{0 . 0 0 1}$ \\
& dv2 & $\mathbf{- 0 . 0 1 6}$ & $\mathbf{0 . 0 0 0}$ & $\mathbf{0 . 0 0 1}$ & $\mathbf{0 . 0 0 0}$ \\
& var & -0.021 & $\mathbf{- 0 . 0 0 3}$ & $\mathbf{- 0 . 0 0 1}$ & $\mathbf{0 . 0 0 1}$ \\
gn & 0.064 & 0.051 & 0.043 & 0.037 \\
& osn & 0.105 & 0.084 & 0.070 & 0.060 \\
& go & -0.036 & -0.028 & -0.021 & -0.016 \\
& oso & $-\mathbf{0 . 0 1 7}$ & -0.014 & -0.011 & -0.009 \\
& ir & -0.043 & -0.013 & -0.014 & -0.013 \\
\hline
\end{tabular}

(a) $H=0.65$

\begin{tabular}{|ccccc|}
\hline$k$ & 8 & 10 & 12 & 14 \\
\hline \hline dv1 & $\mathbf{0 . 0 4 5}$ & $\mathbf{0 . 0 3 0}$ & $\mathbf{0 . 0 1 9}$ & 0.013 \\
dv2 & 0.089 & 0.051 & 0.025 & $\mathbf{0 . 0 1 2}$ \\
var & 0.068 & 0.042 & 0.029 & 0.019 \\
gn & $\mathbf{0 . 0 2 8}$ & $\mathbf{0 . 0 1 5}$ & $\mathbf{0 . 0 0 9}$ & $\mathbf{0 . 0 0 5}$ \\
osn & $\mathbf{0 . 0 3 6}$ & $\mathbf{0 . 0 2 0}$ & $\mathbf{0 . 0 1 2}$ & $\mathbf{0 . 0 0 8}$ \\
go & 0.095 & 0.077 & 0.063 & 0.053 \\
oso & 0.102 & 0.082 & 0.067 & 0.057 \\
ir & 0.170 & 0.095 & 0.038 & 0.021 \\
\hline \hline dv1 & $\mathbf{- 0 . 0 1 6}$ & $\mathbf{- 0 . 0 0 8}$ & $\mathbf{- 0 . 0 0 5}$ & $\mathbf{- 0 . 0 0 3}$ \\
dv2 & $\mathbf{- 0 . 0 1 5}$ & $\mathbf{0 . 0 0 5}$ & $-\mathbf{0 . 0 0 2}$ & $\mathbf{- 0 . 0 0 1}$ \\
var & -0.038 & -0.018 & $-\mathbf{- 0 . 0 1 0}$ & $\mathbf{- 0 . 0 0 5}$ \\
gn & 0.070 & 0.054 & 0.044 & 0.037 \\
osn & 0.110 & 0.086 & 0.071 & 0.061 \\
go & -0.054 & -0.043 & -0.035 & -0.028 \\
oso & -0.033 & -0.027 & -0.022 & -0.018 \\
ir & -0.039 & 0.019 & 0.011 & 0.008 \\
\hline
\end{tabular}

(b) $H=0.85$

Also, if we consider the linear regression $\log (S D) \sim \log (n)$ for these two estimators, its slope is -0.5003 for $\widehat{H}_{d v 2}$ and -0.5013 for $\widehat{H}_{i r}$, which suggests that for both these estimators $S D\left(\widehat{H}_{(\cdot)}\right) \sim \mathcal{O}\left(n^{-1 / 2}\right)$.

\subsection{The geometric Brownian motion}

\subsubsection{Dependance on the value of the Hurst index}

Table 3.3 presents the mean squared errors and the biases for the sample path lengths of $2^{14}+1$ and $2^{10}+1$. Boxplots of these estimators for the sample path length $n=2^{14}+1$ can be found in Figure B.4 (see Appendix). It can be seen that, for the non-Gaussian gBm process, the estimators $\widehat{H}_{g n}$ and $\widehat{H}_{o s n}$ display higher biases for all the values of $H$.

An interesting observation is that, in the case of the O-U process the mean squared errors of $\widehat{H}_{g n}$ and $\widehat{H}_{o s n}$ were the lowest of all the considered estimators, while for the $\mathrm{gBm}$ model their mean squared errors surpassed those of $\widehat{H}_{d v 1}, \widehat{H}_{d v 2}$ and $\widehat{H}_{v a r}$. 
Table 3.3. Comparison of the estimators for the $\mathrm{gBm}$ process.

\begin{tabular}{|c|ccccc|}
\hline \multicolumn{1}{|c}{$H$} & & 0.55 & 0.7 & 0.8 & 0.95 \\
\hline \hline \multirow{5}{*}{ MSE } & dv1 & $\mathbf{0 . 0 0 8}$ & $\mathbf{0 . 0 0 6}$ & $\mathbf{0 . 0 0 9}$ & $\mathbf{0 . 0 2 1}$ \\
& dv2 & $\mathbf{0 . 0 1 6}$ & $\mathbf{0 . 0 1 2}$ & $\mathbf{0 . 0 1 1}$ & $\mathbf{0 . 0 1 0}$ \\
& var & $\mathbf{0 . 0 0 8}$ & $\mathbf{0 . 0 1 0}$ & 0.015 & 0.025 \\
& gn & 0.032 & 0.030 & 0.034 & 0.049 \\
& osn & 0.035 & 0.032 & 0.037 & 0.054 \\
& go & 0.033 & 0.046 & 0.053 & 0.049 \\
& oso & 0.035 & 0.049 & 0.056 & 0.053 \\
& ir & 0.022 & 0.019 & 0.019 & $\mathbf{0 . 0 2 2}$ \\
\hline \hline \multirow{5}{*}{$H$} & dv1 & $\mathbf{0 . 0 0 0}$ & $\mathbf{0 . 0 0 1}$ & $\mathbf{0 . 0 0 0}$ & $\mathbf{- 0 . 0 0 6}$ \\
& dv2 & $\mathbf{0 . 0 0 0}$ & $\mathbf{0 . 0 0 1}$ & $\mathbf{- 0 . 0 0 2}$ & $\mathbf{0 . 0 0 0}$ \\
& var & $\mathbf{0 . 0 0 0}$ & $\mathbf{0 . 0 0 1}$ & $\mathbf{0 . 0 0 0}$ & $\mathbf{- 0 . 0 0 9}$ \\
& gn & 0.055 & 0.051 & 0.061 & 0.059 \\
& osn & 0.080 & 0.076 & 0.086 & 0.082 \\
& go & -0.014 & -0.016 & -0.021 & -0.026 \\
& oso & $-\mathbf{0 . 0 0 3}$ & -0.006 & -0.011 & -0.014 \\
& ir & -0.019 & -0.009 & $-\mathbf{0 . 0 0 1}$ & 0.028 \\
\hline
\end{tabular}

(a) $N=2^{14}+1$

\begin{tabular}{|ccccc|}
\hline$H$ & 0.55 & 0.7 & 0.8 & 0.95 \\
\hline dv1 & $\mathbf{0 . 0 3 0}$ & $\mathbf{0 . 0 2 6}$ & $\mathbf{0 . 0 2 9}$ & $\mathbf{0 . 0 3 0}$ \\
dv2 & 0.054 & 0.054 & 0.055 & $\mathbf{0 . 0 4 6}$ \\
var & $\mathbf{0 . 0 3 0}$ & $\mathbf{0 . 0 3 7}$ & $\mathbf{0 . 0 4 0}$ & $\mathbf{0 . 0 4 1}$ \\
gn & 0.047 & 0.044 & $\mathbf{0 . 0 5 1}$ & 0.079 \\
osn & 0.053 & 0.048 & 0.058 & 0.088 \\
go & 0.055 & 0.072 & 0.080 & 0.066 \\
oso & 0.057 & 0.077 & 0.084 & 0.070 \\
ir & 0.074 & 0.067 & 0.069 & 0.077 \\
\hline dv1 & $\mathbf{- 0 . 0 0 1}$ & $\mathbf{- 0 . 0 0 1}$ & $\mathbf{0 . 0 0 0}$ & $\mathbf{- 0 . 0 1 2}$ \\
dv2 & $\mathbf{0 . 0 0 1}$ & $\mathbf{0 . 0 0 0}$ & $\mathbf{- 0 . 0 0 2}$ & $\mathbf{0 . 0 0 7}$ \\
var & $\mathbf{0 . 0 0 0}$ & -0.006 & -0.008 & -0.020 \\
gn & 0.077 & 0.072 & 0.086 & 0.086 \\
osn & 0.112 & 0.106 & 0.120 & 0.116 \\
go & -0.020 & -0.027 & -0.036 & -0.038 \\
oso & $\mathbf{- 0 . 0 0 1}$ & -0.009 & -0.019 & -0.020 \\
ir & -0.009 & -0.005 & $\mathbf{- 0 . 0 0 4}$ & 0.039 \\
\hline
\end{tabular}

(b) $N=2^{10}+1$

The behavior of $\widehat{H}_{d v 2}$ and $\widehat{H}_{i r}$ does not display notable differences for these two processes.

\subsubsection{Dependance on the length of the sample path}

Table 3.4 presents the mean squared errors and the biases for $H=0.65$ and $H=0.85$. Figure B.5 shows the boxplots of the estimators considered for $H=0.85$, while Figure B.6 presents the plots of $\log (S D)$ against $\log (n)$ (see Appendix).

Compared to the O-U case the biases of $\widehat{H}_{g n}$ and $\widehat{H}_{o s n}$ are higher for all sample path lengths. The mean squared errors of $\widehat{H}_{g n}, \widehat{H}_{o s n}, \widehat{H}_{g o}$ and $\widehat{H}_{o s o}$ are higher for all sample path lengths. In the case of relatively short sample paths $\left(2^{8}-2^{10}\right)$ and $H>3 / 4$, the estimators $\widehat{H}_{g o}$ and $\widehat{H}_{\text {oso }}$ have at times severely overestimated the Hurst index $H$ with the estimated value being higher than 2 . Those values were excluded from their boxplots. The slope of the linear regression $\log (S D) \sim \log (n)$ is -0.5015 for $\widehat{H}_{d v 2}$ and -0.5011 for $\widehat{H}_{i r}$, which does not differ significantly from the $\mathrm{O}-\mathrm{U}$ case. 
Table 3.4. Comparison of the estimators for the gBm process for sample path lengths $N=2^{k}+1$.

\begin{tabular}{|c|ccccc|}
\hline & $k$ & 8 & 10 & 12 & 14 \\
\hline \hline \multirow{5}{*}{$M S E$} & dv1 & $\mathbf{0 . 0 5 2}$ & $\mathbf{0 . 0 2 8}$ & $\mathbf{0 . 0 1 6}$ & $\mathbf{0 . 0 0 7}$ \\
& dv2 & 0.103 & 0.065 & $\mathbf{0 . 0 2 8}$ & $\mathbf{0 . 0 1 3}$ \\
& var & 0.073 & $\mathbf{0 . 0 3 6}$ & $\mathbf{0 . 0 2 0}$ & $\mathbf{0 . 0 1 1}$ \\
gn & $\mathbf{0 . 0 6 6}$ & 0.050 & 0.041 & 0.034 \\
& osn & 0.075 & 0.056 & 0.044 & 0.037 \\
& go & 2.411 & 0.097 & 0.061 & 0.048 \\
& oso & 0.556 & 0.090 & 0.063 & 0.050 \\
& ir & 0.172 & 0.079 & 0.037 & 0.018 \\
\hline \hline \multirow{5}{*}{$H-H 1$} & dve.002 & $\mathbf{0 . 0 0 1}$ & $\mathbf{0 . 0 0 2}$ & $\mathbf{0 . 0 0 1}$ \\
& dv2 & -0.020 & $\mathbf{- 0 . 0 0 2}$ & $\mathbf{0 . 0 0 0}$ & $\mathbf{0 . 0 0 0}$ \\
& var & -0.013 & $\mathbf{0 . 0 0 1}$ & $\mathbf{0 . 0 0 1}$ & $\mathbf{0 . 0 0 2}$ \\
gn & 0.093 & 0.074 & 0.062 & 0.054 \\
& osn & 0.137 & 0.110 & 0.092 & 0.079 \\
& go & 0.223 & -0.009 & -0.011 & -0.010 \\
& oso & 0.065 & 0.006 & $\mathbf{0 . 0 0 1}$ & $\mathbf{0 . 0 0 0}$ \\
& ir & -0.043 & -0.013 & -0.014 & -0.013 \\
\hline
\end{tabular}

\begin{tabular}{|ccccc|}
\hline$k$ & 8 & 10 & 12 & 14 \\
\hline \hline dv1 & $\mathbf{0 . 0 4 7}$ & $\mathbf{0 . 0 3 3}$ & $\mathbf{0 . 0 2 3}$ & $\mathbf{0 . 0 1 6}$ \\
dv2 & 0.094 & $\mathbf{0 . 0 5 3}$ & $\mathbf{0 . 0 2 6}$ & $\mathbf{0 . 0 1 3}$ \\
var & 0.082 & $\mathbf{0 . 0 5 0}$ & $\mathbf{0 . 0 3 2}$ & 0.023 \\
gn & 0.082 & 0.059 & 0.046 & 0.037 \\
osn & 0.092 & 0.065 & 0.049 & 0.040 \\
go & 0.104 & 0.085 & 0.071 & 0.060 \\
oso & 0.109 & 0.090 & 0.075 & 0.064 \\
ir & 0.169 & 0.095 & 0.038 & 0.021 \\
\hline \hline dv1 & $\mathbf{- 0 . 0 0 5}$ & $\mathbf{- 0 . 0 0 1}$ & $\mathbf{0 . 0 0 1}$ & $\mathbf{0 . 0 0 0}$ \\
dv2 & -0.016 & $\mathbf{0 . 0 0 3}$ & $\mathbf{- 0 . 0 0 2}$ & $\mathbf{- 0 . 0 0 1}$ \\
var & -0.025 & -0.007 & $\mathbf{- 0 . 0 0 2}$ & $\mathbf{0 . 0 0 1}$ \\
gn & 0.094 & 0.075 & 0.062 & 0.054 \\
osn & 0.136 & 0.108 & 0.091 & 0.078 \\
go & -0.038 & -0.028 & -0.022 & -0.017 \\
oso & -0.011 & -0.009 & -0.006 & -0.004 \\
ir & -0.039 & 0.019 & 0.011 & 0.008 \\
\hline
\end{tabular}

(a) $H=0.65$

(b) $H=0.85$

\subsection{Conclusions of the modelling}

1. The estimators $\widehat{H}_{g n}$ and $\widehat{H}_{o s n}$, despite showing the least mean squared errors in the O-U case, have also shown much higher biases than other estimators considered in this section. This bias increases as the sample path length is decreased but shows no dependance on the value of the Hurst index $H$ as long as $H$ is not too close to 1 . When $H>0.9$, this bias increases further. In the $\mathrm{gBm}$ case the mean squared errors of these two estimators were greater than those of $\widehat{H}_{d v 1}, \widehat{H}_{d v 2}$ and $\widehat{H}_{v a r}$.

2. The estimators $\widehat{H}_{g o}$ and $\widehat{H}_{\text {oso }}$, the ordinary least squares versions of the previous estimators, display totally different behavior - their biases are comparable with those of the other estimators. However, their mean squared errors are considerably higher than those of other estimators and tend to decrease only slightly as the sample path length is increased. Additionally, both of these estimators require the sample path length to be equal to $2^{k}+1, k \in \mathbb{N}$, which means that, for sample paths of different length, some of the observations must be truncated.

3. The estimators $\widehat{H}_{d v 1}$ and $\widehat{H}_{v a r}$ behaved differently for "small" and "large" values of $H$. As $H \in(1 / 2,3 / 4)$, they displayed the best char- 
acteristics while for higher values of $H$ their performance was close to or worse than that of other estimators. $\widehat{H}_{v a r}$ displayed increased biases for shorter sample paths.

4. The characteristics of $\widehat{H}_{d v 2}$ were slightly worse than that of $\widehat{H}_{d v 1}$ and $\widehat{H}_{v a r}$ for shorter sample paths and $H<3 / 4$, and they were similar or better for longer sample paths and $H>3 / 4$. Also, it showed no notable dependance of its behavior on the value of $H . \widehat{H}_{i r}$ displayed such a dependance only for rather long sample paths, but its biases and mean squared errors were higher. Having considered the linear regression $\log (S D) \sim \log (n)$ for these two estimators, the results suggest that for both these estimators $S D\left(\widehat{H}_{(\cdot)}\right) \sim \mathcal{O}\left(n^{-1 / 2}\right)$.

5. Calculation times for the estimators $\widehat{H}_{d v 1}, \widehat{H}_{d v 2}$ and $\widehat{H}_{o s n}$ were about $0.02 s$ with 100 sample paths of the length $N=2^{8}+1$ and about $0.4 s$ with 100 sample paths of the length $N=2^{14}+1$. Calculation times of $\widehat{H}_{g n}$ were about twice lower and those of $\widehat{H}_{g o}, \widehat{H}_{o s o}$ and $\widehat{H}_{i r}$ were $2-5$ times higher. 



\section{General conclusions}

Having solved the tasks listed in the introduction the following resultes were obtained:

1. Having proved the Theorems $1,3,6$ and 8 , the asymptotics of quadratic variations of the solution of the stochastic differential equation (1.1) were derived both in case of regularly and irregularly spaced observations.

2. In case of regularly spaced observations, in Theorem 2 and Theorem 4 it was proved that $\widehat{H}_{d v 1}^{n}$ and $\widehat{H}_{d v 2}^{n}$, the estimators of the Hurst index $H$ originally obtained by Istas, Lang (1997) and Benassi et al (1998) for the fractional Brownian motion remain strongly consistent when the underlying process is the solution of the stochastic differential equation. In case of irregularly spaced observations, in Theorem 7 it was shown that $\widetilde{H}_{d v 1}^{n}$, the proposed estimator of the Hurst index $H$ based on the first order quadratic variations is strongly consistent.

3. In Theorem 9 it was proved that the obtained estimators remain strongly consistent if the solution of the stochastic differential equation is replaced with its Milstein approximation. 
4. In Theorem 10 it was proved that the increment ratio statistic can be applied to estimate the Hurst index $H$ of the fractional geometric Brownian motion.

5. In Theorem 11 the rate of convergence of the modified Gladyshev estimator of the Hurst index $H$ to its real value was derived.

6. The obtained estimators were compared to some of the other known estimators, namely the naive and ordinary least squares Gladyshev and $\eta$-summing oscillation estimators, the variogram estimator and the IR estimator. The results of the modelling study suggest that if the value of the Hurst index is large $(H>3 / 4)$ or when the Hurst index is estimated from a sufficiently long sample path $\left(N>2^{10}\right)$, the $\widehat{H}_{d v 2}$ estimator performs best. If either of these assumptions is not present, then $\widehat{H}_{d v 1}$ and $\widehat{H}_{v a r}$ would likely provide a more precise estimate. 


\section{References}

Bardet, J. M.; Surgailis, D. 2010. Measuring the roughness of random paths by increment ratios, Bernoulli: preprint. ISSN 1350-7265.

Bégyn, A. 2005. Quadratic Variations along Irregular Subdivisions for Gaussian Processes, Electronic Journal of Probability 10: 691-717. ISSN 1083-6489.

Bégyn, A. 2006. Generalized Quadratic Variations of Gaussian Processes: Limit Theorems and Applications to Fractional Processes: Doctoral Dissertation. Université Toulouse III - Paul Sabatier. Toulouse: VDM Verlag. 168 p. ISBN 9783639188004.

Benassi, A.; Cohen, S.; Istas, J.; Jaffard, S. 1998. Identification of filtered white noises, Stochastic Processes and their Applications 75: 31-49. ISSN 0304-4149.

Bertrand, P. R.; Fhima, M.; Guillin, A. 2011. Fast change point analysis on the Hurst index of piecewise fractional Brownian motion, in Acte des Journees de Statistiques.

Berzin, C.; León, J. 2008. Estimation in models driven by fractional Brownian motion, Annales de l'Institut Henri Poincare - Probabilites et Statistiques 44(2): 191213. ISSN 0246-0203.

Coeurjolly, J. F. 2000. Simulation and identification of the fractional Brownian motion: a bibliographical and comparative study, Journal of Statistical Software 5(7): 153. ISSN 1548-7660. 
Coeurjolly, J. F. 2001. Estimating the parameters of a frational Brownian motion by discrete variations of its sample paths, Statistical Inference for Stochastic Processes 4(2): 199-227. ISSN 1572-9311.

Chronopoulou, A.; Viens, F. G. 2010. Simulation and identification of the fractional Brownian motion: a bibliographical and comparative study, Recent Advances in Stochastic Dynamics and Stochastic Analysis 8: 91-119. ISBN 981-4277-25-8.

Davis, G. 1996. Implicit Image Models in Fractal Image Compression, in Proceedings of the SPIE conference on Wavelet Applications in Signal and Image Processing IV , vol. 2825, 88-97.

Decreusefond, L.; Üstünel, A. S. 1995. Application du calcul des variations stochastiques an mouvement brownien fractionnaire, Comptes rendus de l'Academie des sciences 321: 1605-1608. ISSN 0764-4442.

Dudley, R. M. 1999. Picard iteration and p-variation: the work of Lyons, in MiniProceedings: Workshop on Product Integrals and Pathwise Integration.

Gine, E.; Klein, R. 1975. On quadratic variations of processes with Gaussian increments, The Annals of Probability 3(4): 716-721. ISSN 0091-1798.

Gladyshev, E. G. 1963. A new limit theorem for stochastic processes with Gaussian increments, Theory of Probability and Its Applications 6(1): 52-61. ISSN 1095-7219.

Gushchin, A. A.; Kuchler, U. 2005. On recovery of a measure from its symmetrization, Theory of Probability and Its Applications 49: 323-333. ISSN 1095-7219.

Hanson, D. I.; Wright, F. T. 1971. A bound on tail probabilities for quadratic forms in independent random variables, Annals of the Institute of Statistical Mathematics 42: 1079-1083. ISSN 0020-3157.

Hurst, H. 1951. Long Term Storage Capacity of Reservoirs, Transactions of the American Society of Civil Engineers 116: 770-799.

Istas, J.; Lang, G. 1997. Quadratic variations and estimation of the local Hölder index of a Gaussian process, Annales de l'Institut Henri Poincare - Probabilites et Statistiques 33: 407-436. ISSN 0246-0203.

Kubilius, K. 1999. An approximation of a non-linear integral equation driven by a function of bounded p-variation, Lithuanian Mathematical Journal 39(3): 317-330. ISSN 0363-1672.

Kubilius, K. 2000. The existence and uniqueness of the solution of the integral equation driven by the fractional Brownian motion, Lithuanian Mathematical Journal 40(spec.nr.): 104-110. ISSN 0363-1672.

Kubilius, K. 2002. On the asymptotic behavior of an approximation of SIEs driven by $p$-semimartingales, Mathematical Modelling and Analysis 7(1): 103-116. ISSN 1648-3510. 
Li, M. 2010. Essay on teletraffic models (I), in Proceedings of the 9th WSEAS international conference on Applied computer and applied computational science, 130 135 .

Liptser, R. Sh.; Shiryaev, A. N. 1989. Theory of Martingales. Dordrecht: Springer. 1-st edition. 812 p. ISBN 978-0792303954.

Lyons, T. 1994. Differential equations driven by rough signals (I): An extension of an inequality of L.C. Young, Mathematical Research Letters 1: 451-464. ISSN 10732780.

Mandelbrot, B. B.; van Ness, J. W. 1968. Fractional Brownian motions, fractional noices and applications, SIAM Review 10: 422-437. ISSN 0036-1445.

Mandelbrot, B. B. 1995. Fractals and Scaling In Finance: Discontinuity, Concentration, Risk. New York: Springer. 564 p. ISBN 978-1441931191.

McLeish, D. L. 1978. An extended martingale invariance principle, The Annals of Probability 6(1): 144-150. ISSN 0091-1798.

Norvaiša, R.; Salopek, D. M. 2002. Estimating the $p$-variation index of a sample function: An application to financial data set, Methodology and Computing in Applied Probability 4(1): 27-53. ISSN 1573-7713.

Nualart, D.; Răşcanu, A. 2002. Differential equations driven by fractional Brownian motion, Collectanea mathematica 53(1): 55-81. ISSN 0010-0757.

R Development Core Team. 2009. R: A Language and Environment for Statistical Computing. Vienna, Austria. ISBN 3-900051-07-0, URL http://www.R-project.org.

Shao, Q.-M. 1996. $p$-variation of Gaussian processes with stationary increments, Studia Scientiarum Mathematicarum Hungarica 31: 237-247. ISSN 0081-6906.

Sottinen, T.; Valkeila, S. 2003. On arbitrage and replication in the fractional Black-Scholes pricing model, Statistics and Decisions 21: 137-151. ISSN 0721-2631.

Taqqu, M. 1975. Weak convergence to fractional Brownian motion and to the Rosenblatt process, Z. Wahrscheinlichkeitstheorie verw. Gebiete 31: 287-302.

Wenlu, X.; Weixin, X. 1997. Image object detection based on fractional Brownian motion, Journal of Electronics 14(4): 289-294. ISSN 1993-0615. 



\section{List of author's publications on the topic of dissertation}

\section{In the reviewed scientific journals}

Kubilius, K.; Melichov, D. 2011. On comparison of the estimators of the Hurst index of the solutions of stochastic differential equations driven by the fractional Brownian motion, Informatica 22(1): 97-114. ISSN 0868-4952 (Thomson ISI Web of Science).

Kubilius, K.; Melichov, D. 2010. On the convergence rates of Gladyshev's Hurst index estimator, Nonlinear analysis: modelling and control 15(4): 445-450. ISSN 13925113 (Thomson ISI Web of Science).

Kubilius, K.; Melichov, D. 2010. Quadratic variations and estimation of the hurst index of the solution of SDE driven by a fractional Brownian motion, Lithuanian mathematical journal 50(4): 401-417. ISSN 0363-1672 (Thomson ISI Web of Science).

Melichov, D. 2010. Applying the IR statistic to estimate the Hurst index of the fractional geometric Brownian motion, Lietuvos matematikos rinkinys. LMD darbai 51: 368-372. ISSN 0132-2818.

Kubilius, K.; Melichov, D. 2009. Estimating the Hurst index of the solution of a stochastic integral equation, Lietuvos matematikos rinkinys. LMD darbai 50: 24-29. ISSN 0132-2818.

Kubilius, K.; Melichov, D. 2008. On estimation of the Hurst index of solutions of stochastic integral equations, Lietuvos matematikos rinkinys. LMD darbai 48/49: 401406. ISSN 0132-2818. 



\section{Appendices}

\section{Appendix A. [R] source codes}

\section{Appendix A.1. Generation of the fractional Brownian motion}

The function $\operatorname{genFBM}(H, N)$ generates a single sample path of the $\mathrm{fBm}$ with the Hurst index $H$ of the length $N$ data points over the unit interval.

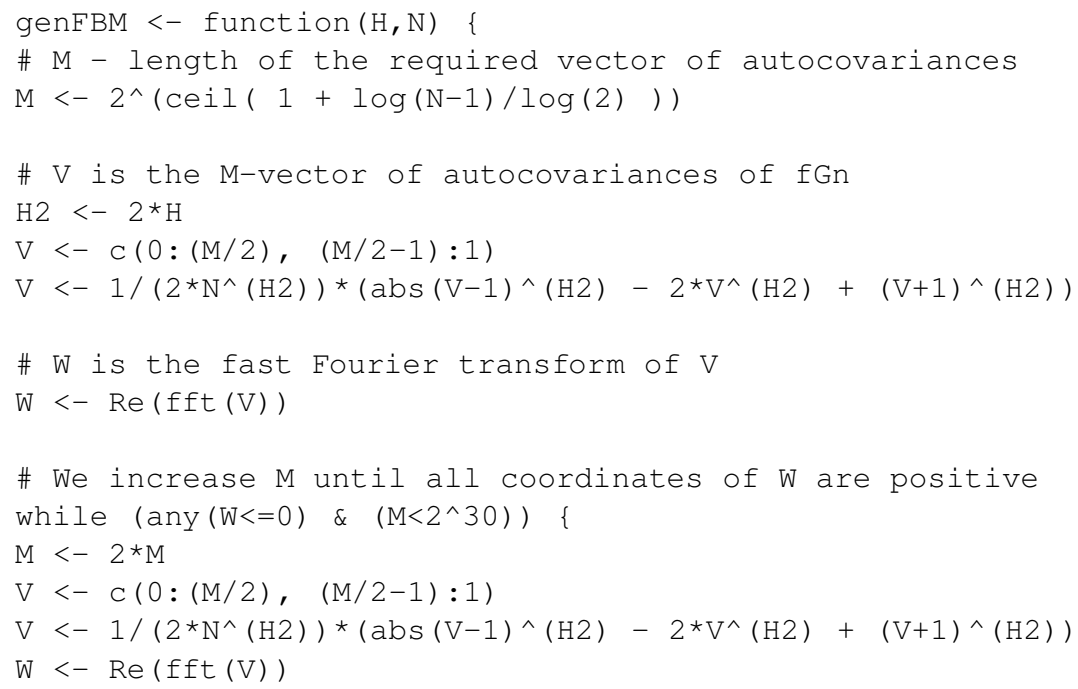




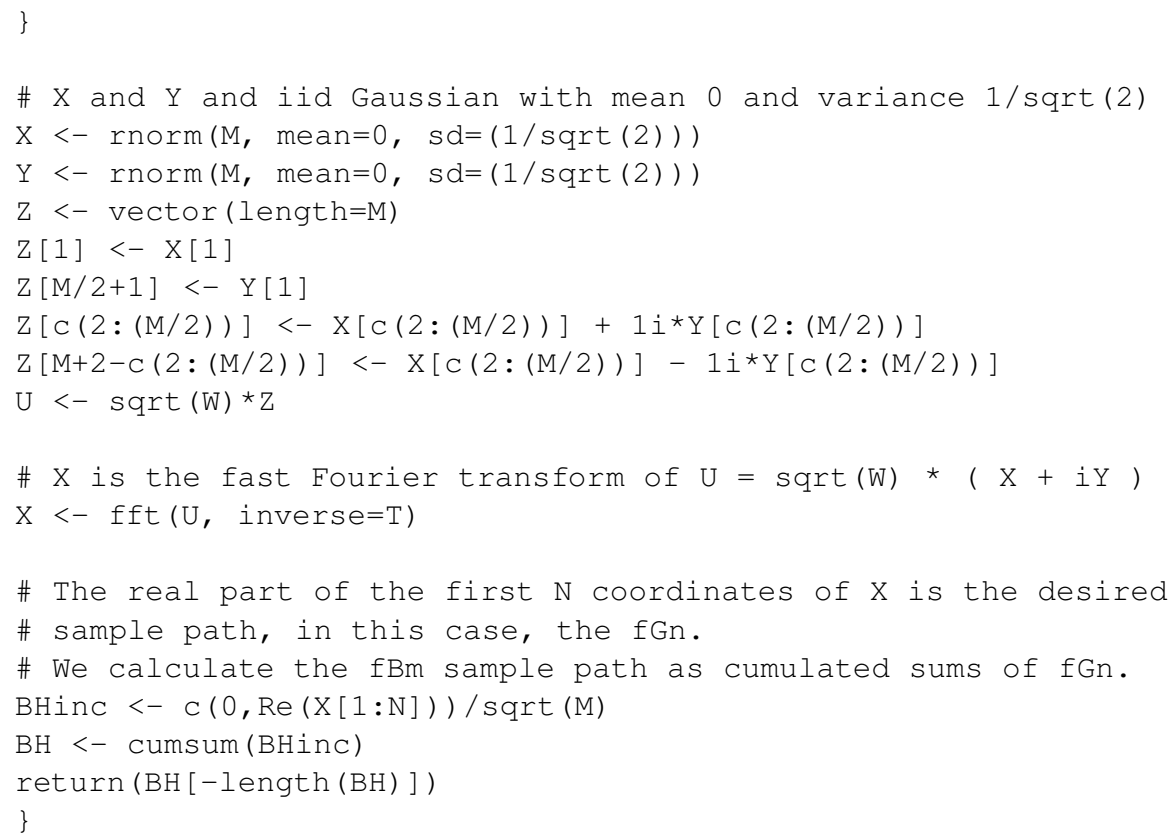

\section{Appendix A.2. Calculation of the estimators}

The following functions estimate the value of the Hurst index $H$ of a single sample path.

fH1 calculates the $\widehat{H}_{d v 1}^{n}$ estimate:

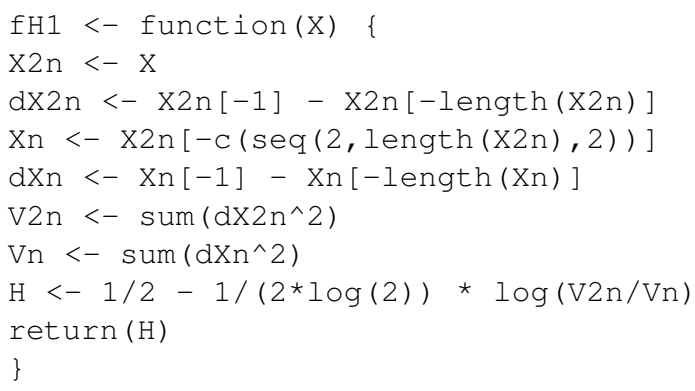

fH2 calculates the $\widehat{H}_{d v 2}^{n}$ estimate:

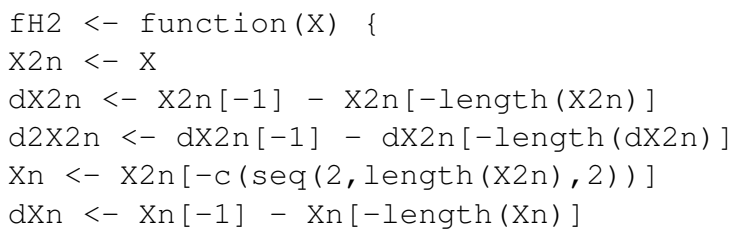




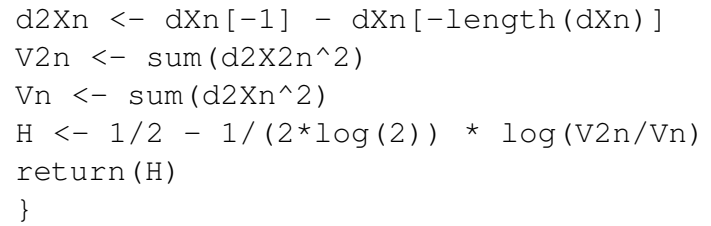

IR calculates the $\widehat{H}_{i r}^{n}$ estimate:

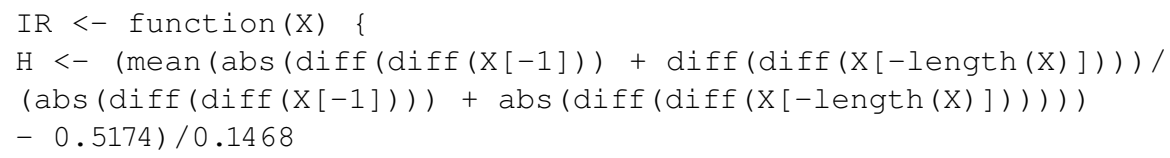

G_naive calculates the $\widehat{H}_{g n}^{n}$ estimate:

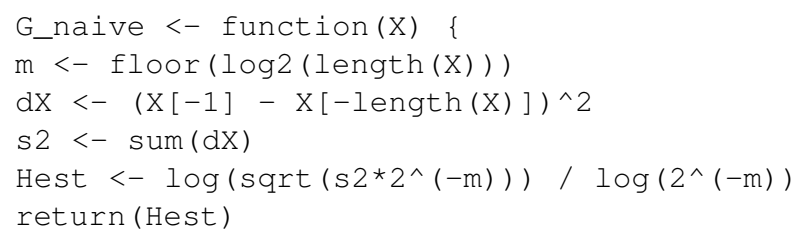

OS_naive calculates the $\widehat{H}_{o s n}^{n}$ estimate:

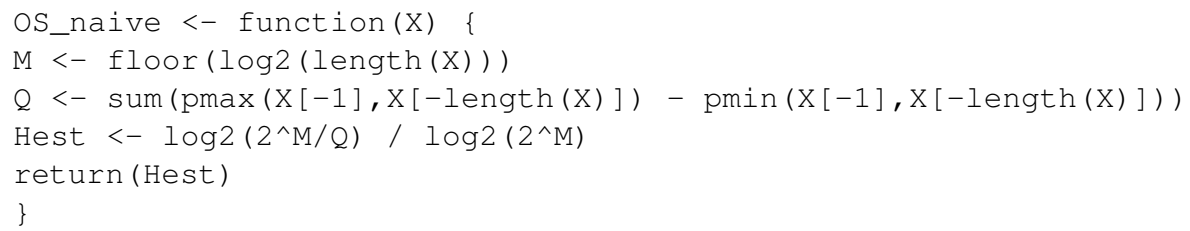


OS_OLS calculates the $\widehat{H}_{\text {oso }}^{n}$ estimate:

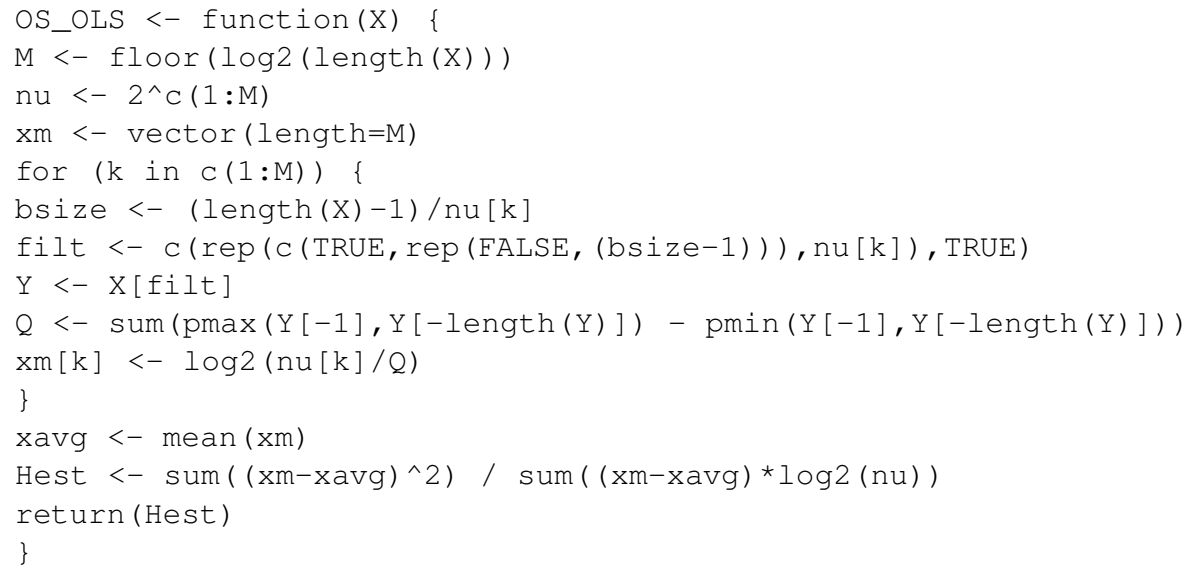

Hvar calculates the $\widehat{H}_{v a r}^{n}$ estimate:

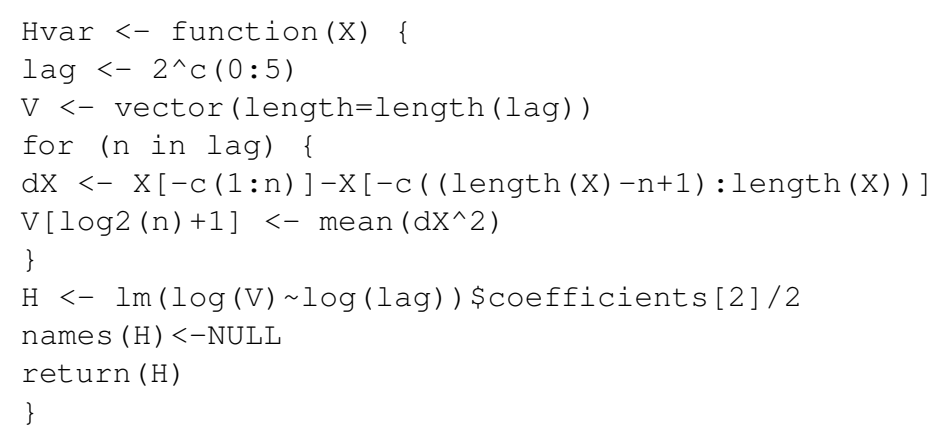




\section{Appendix B. Figures}
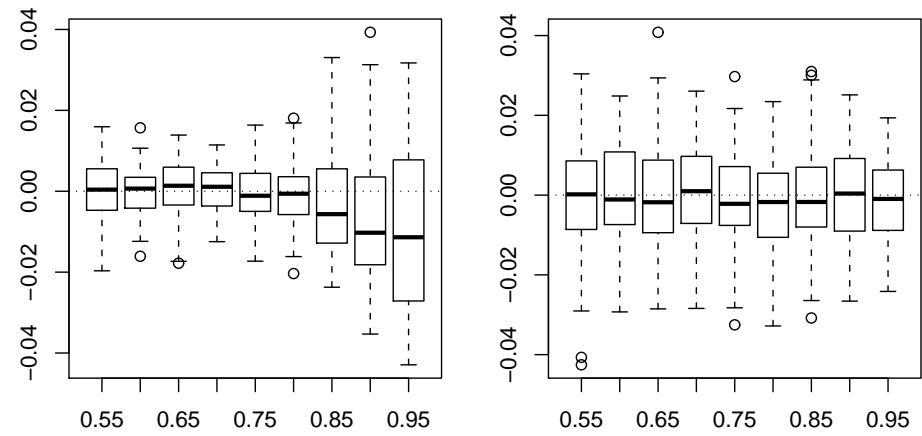

(a) dv 1

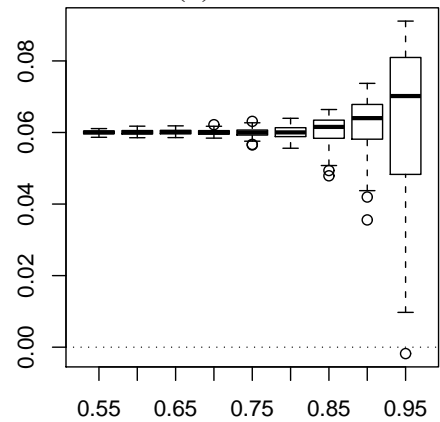

(b) dv2

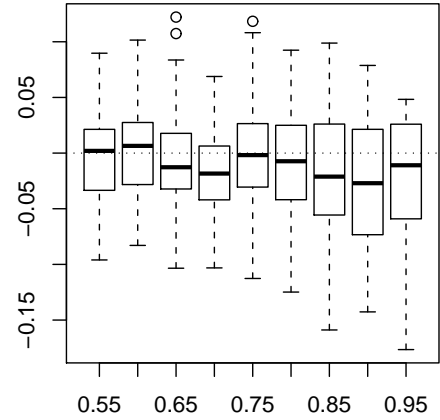

(c) osn

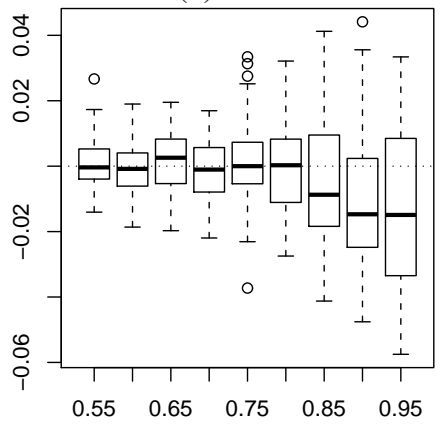

(d) oso

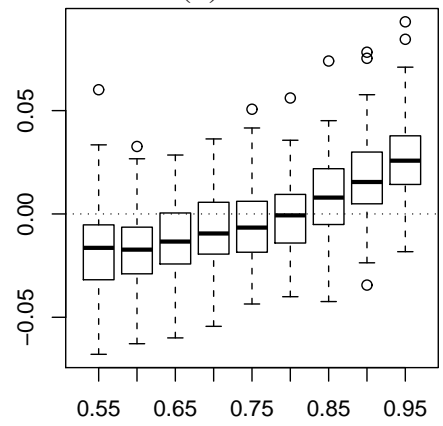

(e) var

(f) ir

Fig. B.1. Boxplots for the O-U process, sample path length $n=2^{14}+1$. 


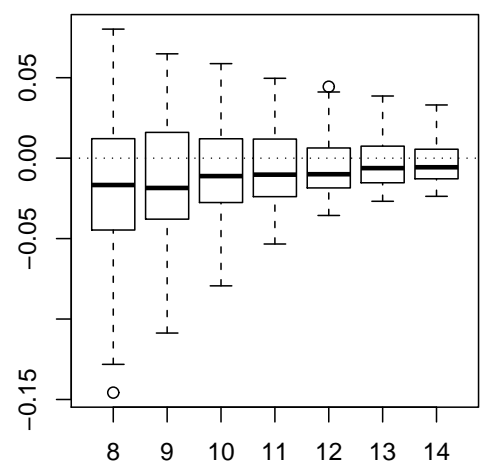

(a) dv1

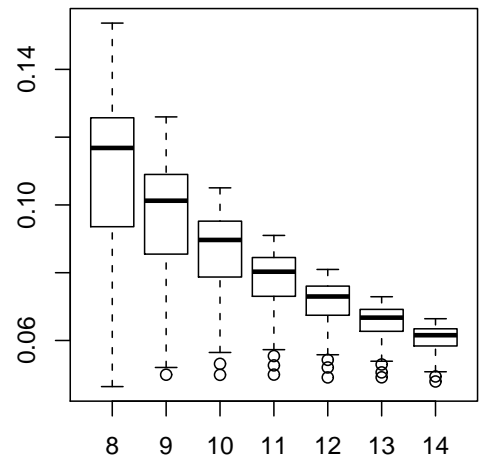

(c) osn

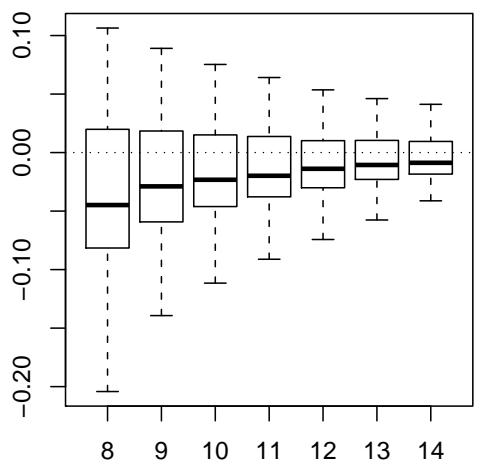

(e) var

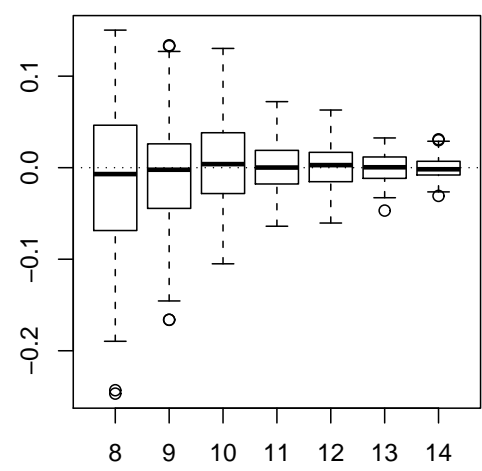

(b) dv2

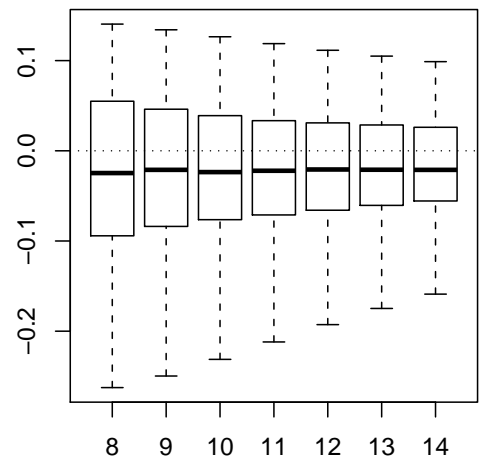

(d) oso

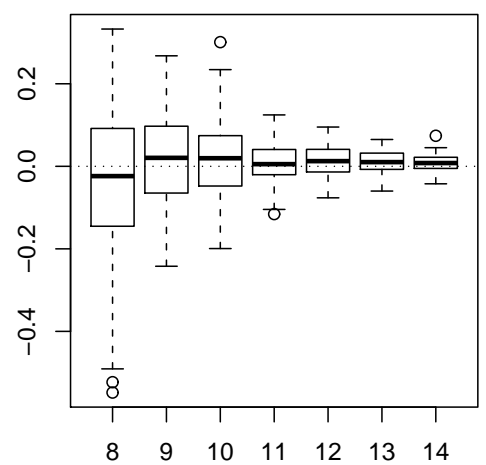

(f) $\mathrm{ir}$

Fig. B.2. Boxplots for the O-U process, $H=0.85, n=2^{k}, k=8, \ldots, 14$. 


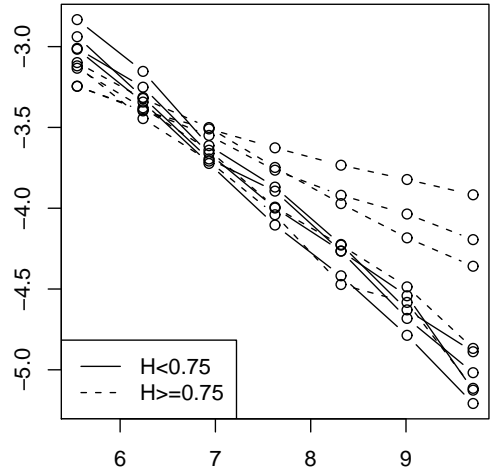

(a) dv1

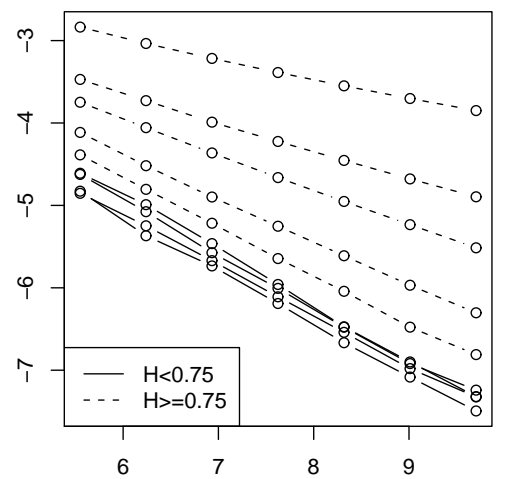

(c) osn

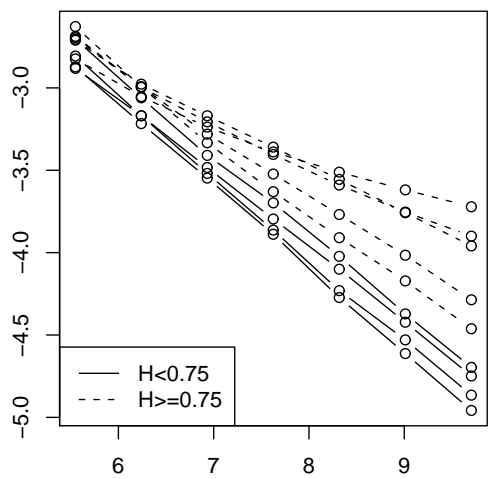

(e) var

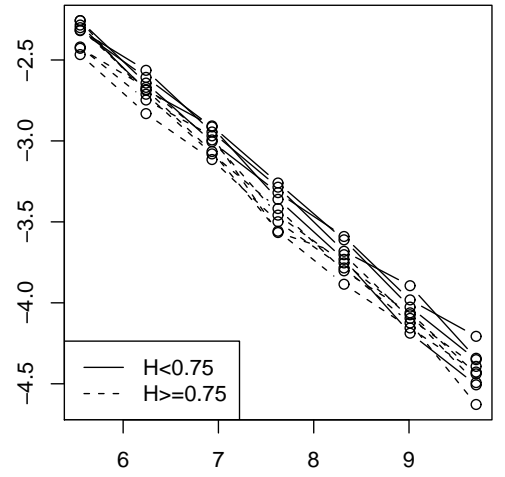

(b) dv2

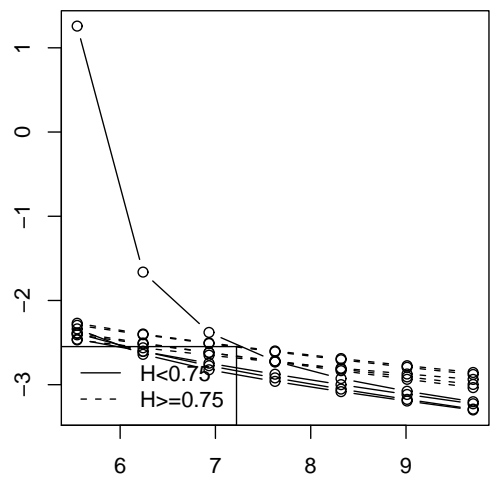

(d) oso

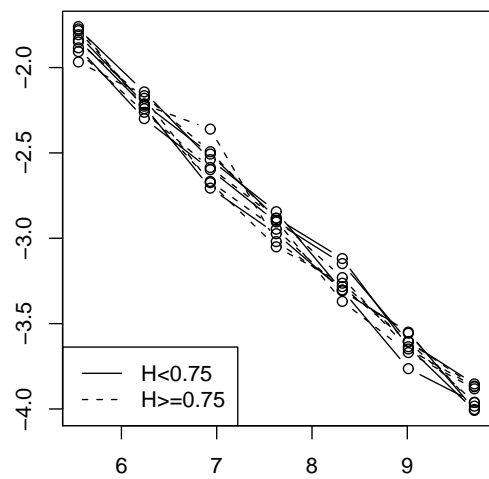

(f) ir

Fig. B.3. Dependance of $\log (S D)$ against $\log (n)$ for the O-U process, $H \in\{0.55,0.6, \ldots, 0.95\}$. 


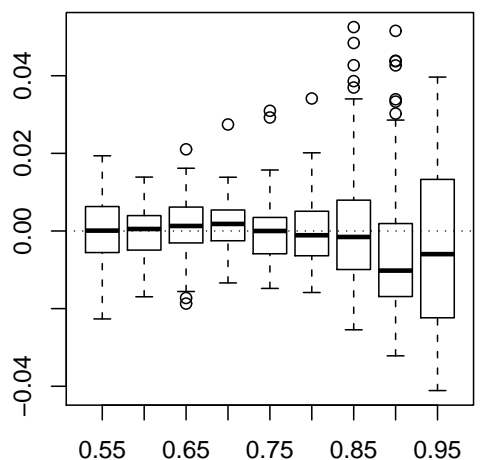

(a) dv 1

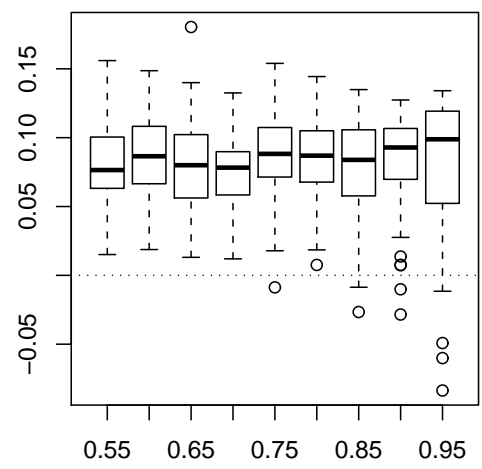

(c) osn

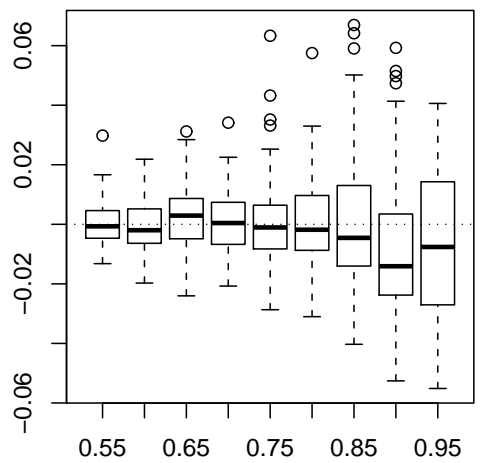

(e) var

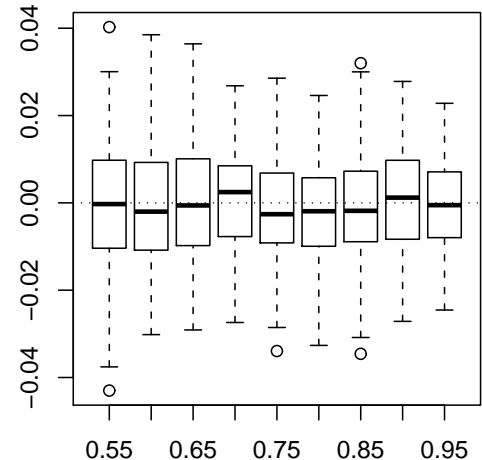

(b) dv2

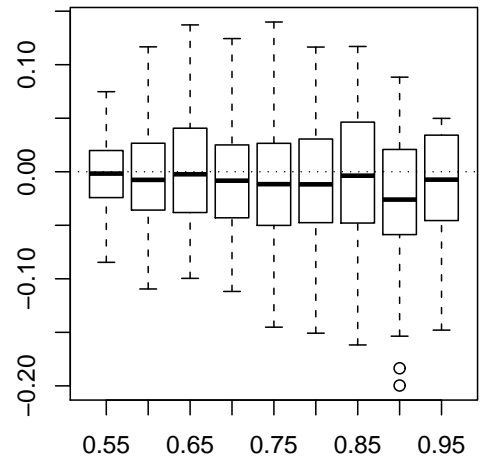

(d) oso

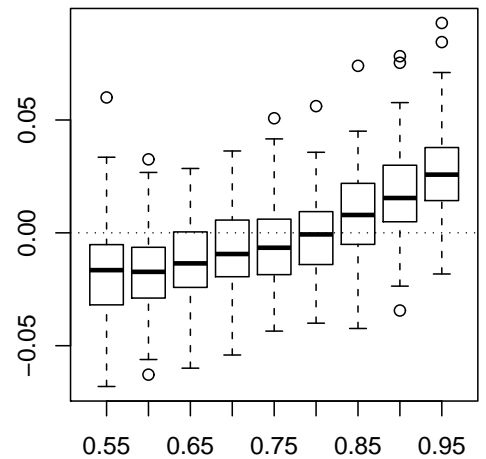

(f) ir

Fig. B.4. Boxplots for the B-S process, sample path length $n=2^{14}+1$. 


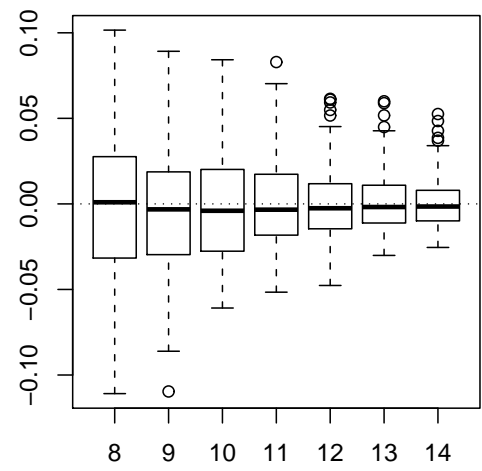

(a) dv1

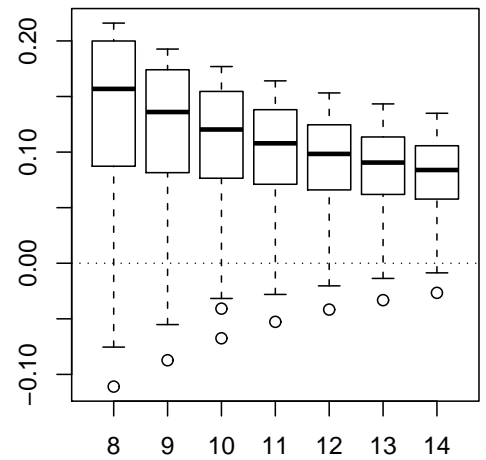

(c) osn

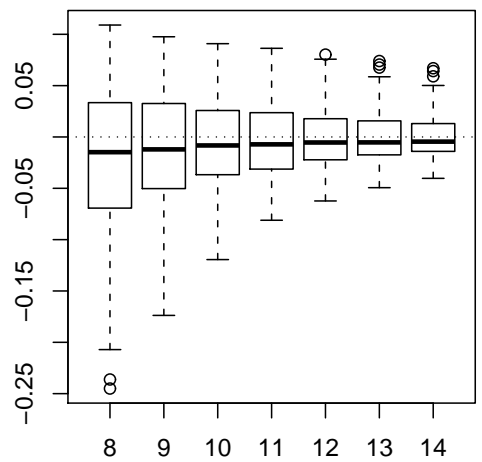

(e) var

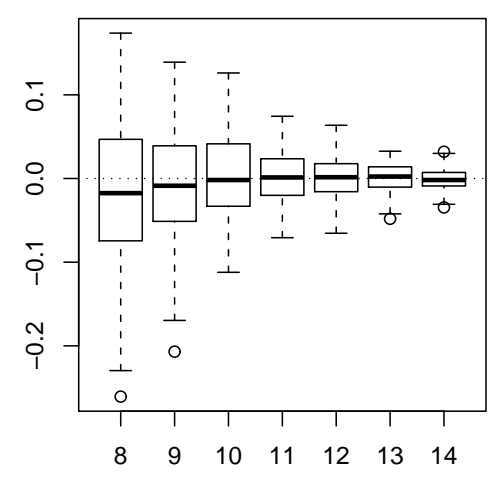

(b) dv2

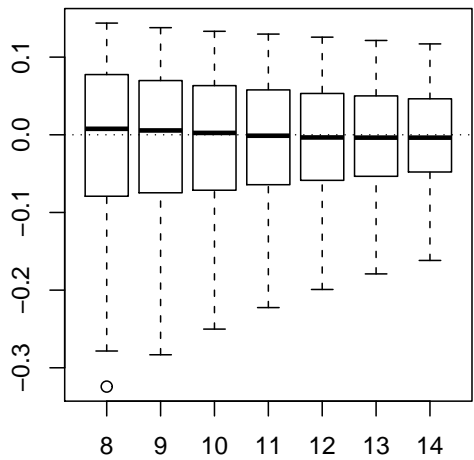

(d) oso

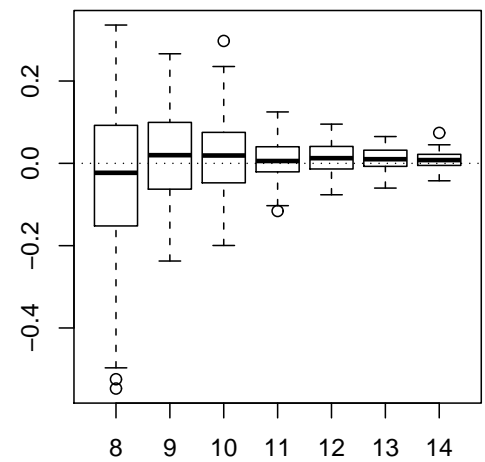

(f) ir

Fig. B.5. Boxplots for the B-S process, $H=0.85, n=2^{k}, k=8, \ldots, 14$. 


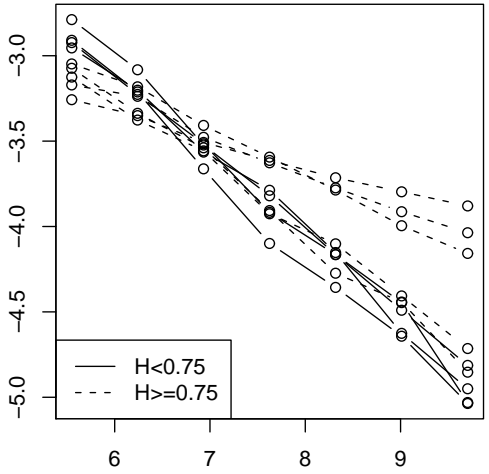

(a) dv1

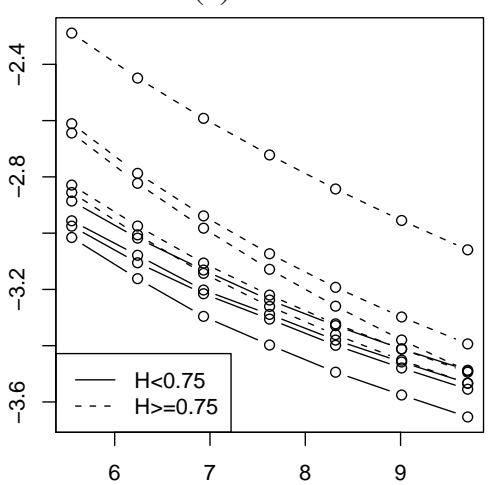

(c) osn

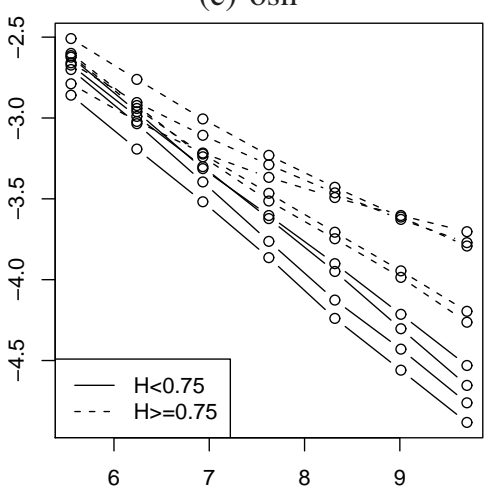

(e) var

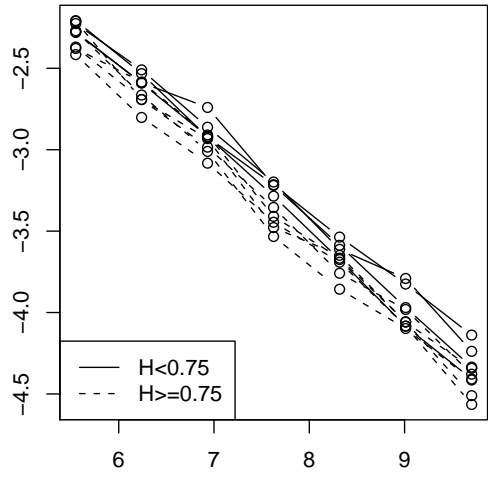

(b) dv2

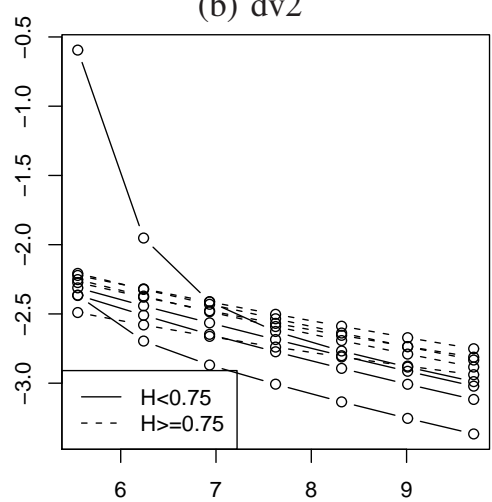

(d) oso

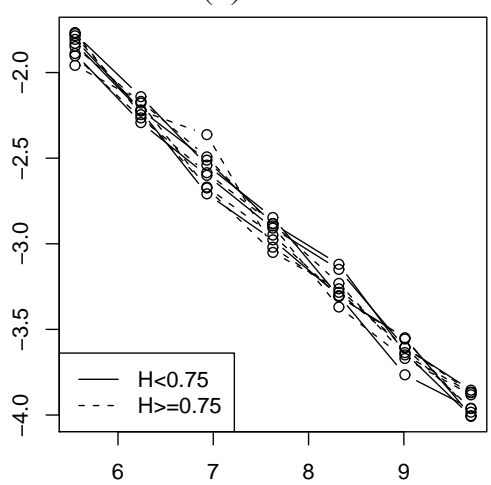

(f) ir

Fig. B.6. Dependance of $\log (S D)$ against $\log (n)$ for the B-S process, $H \in\{0.55,0.6, \ldots, 0.95\}$. 
Dmitrij MELICHOV

ON ESTIMATION OF THE HURST INDEX

OF SOLUTIONS OF STOCHASTIC

DIFFERENTIAL EQUATIONS

Doctoral Dissertation

Physical Sciences, Mathematics (01P)

APIE STOCHASTINIU DIFERENCIALINIU

LYGČIU SPRENDINIU

HURSTO INDEKSO VERTINIMA

Daktaro disertacija

Fiziniai mokslai, Matematika (01P)

201111 03. 7,5 sp. 1. Tiražas 20 egz.

Vilniaus Gedimino technikos universiteto leidykla „Technika“,

Saulètekio al. 11, LT-10223 Vilnius, http://leidykla.vgtu.lt

Spausdino UAB „Ciklonas“,

J. Jasinskio g. 15, LT-01111 Vilnius 